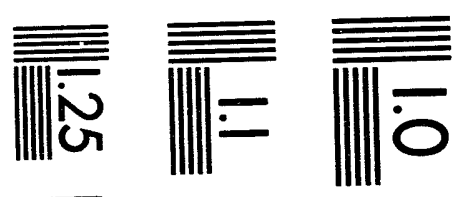

$$
\begin{aligned}
& \text { 需市 } \\
& \text { IIIII }
\end{aligned}
$$

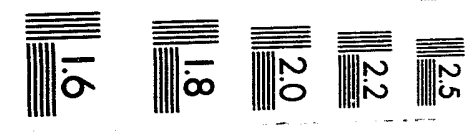



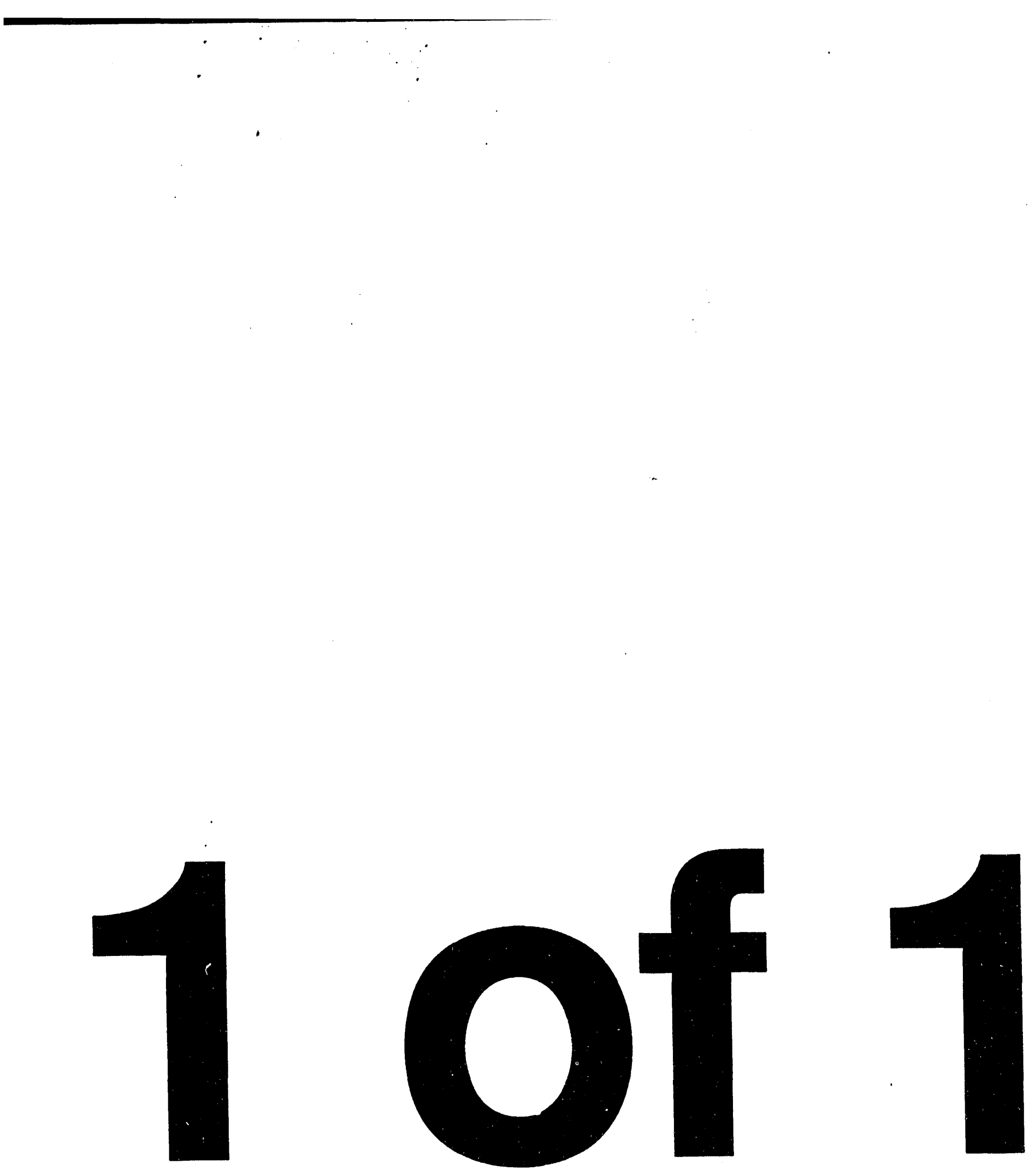


\title{
Location Capability of a Sparse Regional Network (RSTN) using a Multi-phase Earthquake Location Algorithm (REGLOC)
}

\author{
Lawrence Hutchings
}

January 1994

This is an informal report intended primarily for internal or limited external distribution. The opinions and conclusions stated are those of the author and may or may not be those of the Laboratory.

Work performed under the auspices of the U.S. Department of Energy by the Lawrence Livermore National Laboratory under Contract W-7405-Eng-48. 


\section{DISCLAIMER}

This document was prepared as an account of work sponsored by an agency of the United States Government. Neither the United States Government nor the University of California nor any of their employees, makes any warranty, express or implied, or assumes any legal liability or responsibility for the accuracy, completeness, or usefulness of any information, apparatus, product, or process disclosed, or represents that its use would not infringe privately owned rights. Reference herein to any specific commercial products, process, or service by trade name, trademark, manufacturer, or otherwise, does not necessarily constitute or imply its endorsement, recommendation, or favoring by the United States Government or the University of California. The views and opinions of authors expressed herein do not necessarily state or reflect those of the United States Government or the University of California, and shall not be used for advertising or product endorsement purposes.

This report has been reproduced directly from the best available copy.

Available to DOE and DOE contractors from the Omice of Scientific and Technical Information

P.O. Box 62, Oak Ridge, TN 37831

Prices available from (615) 576-8401, FTS 626-8401

A vailable to the public from the

National Technical Information Service

U.S. Department of Commerce

5285 Port Royal Rd.

Springfield, VA 22161 


\title{
Location Capability of a Sparse Regional Network (RSTN) using a Multi-phase Earthquake Location Algorithm (REGLOC)
}

\begin{abstract}
The Regional Seismic Tesi Network (RSTN) was deployed by the U.S. Department of Energy (DOE) to determine whether data recorded by a regional network could be used to detect and accurately locate seismic events that might be clandestine nuclear tests. The purpose of this paper is to evaluate the location capability of the RSTN. A major part of this project was the development of the location algorithm REGLOC and application of Basian a prior statistics for determining the accuracy of the location estimates. REGLOC utilizes all identifiable phases, including backazimuth, in the location. Ninty-four events, distributed throughout the network area, detected by both the RSTN and located by local networks were used in the study.

The location capability of the RSTN was evaluated by estimating the location accuracy, error ellipse accuracy, and the percentage of events that could be located, as a function of magnitude. The location accuracy was verified by comparing the RTSN results for the 94 events with published locations based on data from the local networks. The error ellipse accuracy was evaluated by determining whether the error ellipse (at the 95 and $99 \%$ confidence levels) includes the actual location. The percentage of events located was assessed by combining detection capability with location capability to determine the percentage of events that could be located within the study area. It is estimated that $100 \%$ of events above magnitude 3.0 could be detected and located with the RSTN.

Events were located with both an average crustal model for the entire region, and with regional velocity models along with station corrections obtained from master events. Most events with a magnitude $<3.0$ can only be located with arrivals from one station. Their average location errors are 453 and $414 \mathrm{~km}$ for the average- and regionalvelocity model locations, respectively. Single station locations are very unreliable because they depend on accurate backazimuth estimates, and backazimuth proved to be a very unreliable computation. This is partially due to the fact that only small events required a single station location and that these also had a very poor SNR. However, even events with high SNRs have a backazimuth error of $\pm 15^{\circ}$. Considering that the location error linearly increases with distance when using backazimuth estimates, this too suggests that backazimuth is a very poor measurement to use.

Fifty percent of the events with magnitudes of 3.0-3.5 are located with two stations. The average location errors when locating with two stations are 85 and $68 \mathrm{~km}$ for the average- and regional-velocity model locations, respectively. Locations with two stations are almost as accurate as locations with three or more stations; this is because backazimuth is not primary to the location computation, but only acts to distinguish between ambiguous interpretations. Ninety percent of the events above a magnitude of 3.5 are located with three or more stations. Their average location errors are 73 and $35 \mathrm{~km}$, for the average- and regional-velocity model locations, respectively. The average location accuracy for events above a magnitude of 3.5 does not depend on magnitude.

Fifty-six percent of the events located with the average crustal model and $82 \%$ of the events located with regional velocity models have locations that fall within the $95 \%$ confidence error ellipses. Error ellipse estimates only account for the random errors in location. This suggests that including regional velocity models and calibrated travel times from master events does not account for all of the systematic errors. Most error ellipses that do not include the actual location of events are close to the event. Seventy-five and $86 \%$ of events fall within the $99 \%$ confidence error ellipses for the average and regional crustal models, respectively. For purposes of test ban treaty verification it is concluded that $100 \%$ of events above magnitude 3.0 could be detected and located with accuracy less than $68 \mathrm{~km}$, and $99 \%$ error ellipses calculated are reliable at the $86 \%$ confidence level. Events below magnitude 3.0 cannot be fully detected and their location accuracy is fairly poor.
\end{abstract}

$$
\text { MASTER }
$$




\section{Introduction}

Seismic data can be used to monitor and verify treaties limiting or banning nuclear tests. The Regional Seismic Test Network (RSTN) was deployed by the U.S. Department of Energy (DOE) to determine whether data recorded by a regional network could be used to detect and accurately locate seismic events that might be clandestine nuclear tests. The RSTN was established as part of the U.S. Department of Energy's Test Ban Treaty Verification Program as a prototype of a network that could be installed in the former Soviet Union to monitor a Comprehensive Test Ban Treaty (CTBT) or Threshold Test Ban Treaty (TTBT). Monitoring for CTBT or TTBT will require evaluating approximately 50,000 seismic events with a magnitude $M \geq 2.5$ each year; one technique that could drastically reduce this computational effort is a discrimination method based on location. The RSTN was configured to detect possible clandestine nuclear tests and to provide data for preliminary discrimination based on location. However, to reliably monitor these treaties, the detection and location capability of the monitoring network must be well defined; the purpose of this study was to evaluate the usefulness (or location capability) of RSTN data by estimating the location accuracy, error ellipse accuracy, and the percentage of events located as a function of magnitude. The techniques developed for this study can also be applied to more conventional earthquake studies and may be useful in containing the proliferation of nuclear weapons.

The location accuracy is verified by comparing the RTSN results for 94 events to published locations based on data from local networks. These 94 events were distributed throughout the RSTN area. The error ellipse accuracy is evaluated by determining whether the error ellipse at the 95 and $99 \%$ confidence levels includes the actual location. The error ellipse reliability is more important to test ban treaty verification than the location estimate. Even when the location is not accurate, if it can be reliability assumed that an event occurred within an error ellipse, then discrimination based on location can be a very powerful technique. The percentage of events that can be located is assessed by combining detection capability with location capability to determine the percentage of events that could be located within the study area.

The RSTN consists of five broadband, three-component, seismic stations located in the United States and Canada: Tennessee (RSCP), Northwest Territories (RSNT), New York (RSNY), Ontario (RSON), and South Dakota (RSSD) (Figure 1; Table 1). Stations are distributed across North America in an area and geology roughly similar to that of the former Soviet Union. The RSTN system records signals from seismometers located in 100-m boreholes. After signal processing (Nakanishi et al., 1983), the instrument response is removed to obtain a broadband velocity that is flat between 0.5 and $16 \mathrm{~Hz}$. A 40-sps digitized record of each event is saved for analysis.

For this study, the location capability is initially evaluated for an average earth structure for North America without station corrections-a situation that might exist during the early stages of monitoring. Then, the location capability is evaluated for specific regional velocity models with station corrections obtained from master events-a situation that would be possible after a period of operation. For very sparse data, Baysian a priori statistics are implemented to estimate travel-time variance and confidenceerror ellipses. Further, statistics are developed for each individual phase at each station, and for each velocity model used. From these results, the most accurate error ellipse possible is estimated.

Because of the sparse station distribution, most events with magnitudes $<3.0$ are located with arrivals from one or two stations. For example, the only information available for a location may be the $P_{\mathcal{E}}$ and $S_{n}$ phases and the backazimuth from one station $500 \mathrm{~km}$ distant, and the $L_{g}$ phase and backazimuth 
from an additional station $2000 \mathrm{~km}$ distant. The algorithm (REGLOC) was developed to address a scenario in which earthquakes must be located with very sparse data. REGLOC utilizes multiple phases and backazimuth in an iterative least-squares location inversion. This, with signal processing to maximize the number of phases and backazimuths obtained, minimizes the magnitude threshold of location and maximizes the accuracy of location. At regional distances, identifiable phases include $P_{n}, S_{n}, P_{g}$, and $L_{g}$. Additional phases can be easily added to the location algorithm if necessary. Utilizing multiple phases also reduces inaccuracies that result from random errors. The $L_{g}$ phase is a trapped higher mode Love-Rayleigh wave combination that arrives at the $S_{8}$ wave velocity.

\section{Location Algorithm}

The generalized linear least-squares inversion location technique (Geiger, 1910; Lee and Lahr, 1971 ) is modified to include mixed-mode observational data (Jackson, 1972; Foy, 1975). Here, mixed-mode indicates arrival times and backazimuths. In addition, range estimates, as dependent on two arrival times, are automatically included. The basic mathematical principles are outlined below.

The observed $i^{\text {th }}$ arrival time or backazimuth is $\tau_{i}$, and the calculated value is $t_{i}$. The solution that minimizes the residuals

$$
R_{i}=\tau_{i}-t_{i}\left(\phi_{o}, \lambda_{o}, z_{o}, t_{o}, \phi_{s}, \lambda_{s}\right)
$$

is sought. Subscript $s$ refers to station values, and $\phi_{0}, \lambda_{0}, z_{0}$, and $t_{0}$ are the true latitude, longitude, depth and origin time, respectively (henceforth referred to only as true location). The true location is expressed as a trial location $(\phi, \lambda, z, t)$ plus an error $(\delta \phi, \delta \lambda, \delta z, \delta t)$ called the adjustment vector:

$$
\begin{aligned}
& \phi_{0}=\phi+\delta \phi \\
& \lambda_{0}=\lambda+\delta \lambda \\
& z_{0}=z+\delta z \\
& t_{0}=t+\delta t .
\end{aligned}
$$

The value $t_{i}$ is expanded in a Taylor series about the trial location.

$$
t_{i}=\dot{t}_{i}+\frac{\partial t_{i}}{\partial \phi} \delta \phi+\frac{\partial t_{i}}{\partial \lambda} \delta \lambda+\frac{\partial t_{i}}{\partial z} \delta z+\frac{\partial t_{i}}{\partial t} \delta t+\varepsilon_{i}^{\prime}
$$

where $t_{i}^{\prime}$ is the calculated travel time from the trial location, and $\varepsilon_{i}^{\prime}$ are higher order terms. The error is expressed as:

$$
\varepsilon_{i}=\tau_{i}-t_{i}+\varepsilon_{i}^{\prime}=R_{i}^{\prime}-\frac{\partial t_{i}}{\partial \phi} \delta \phi-\frac{\partial t_{i}}{\partial \lambda} \delta \lambda-\frac{\partial t_{i}}{\partial z} \delta z-\frac{\partial t_{i}}{\partial t} \delta t
$$

where $R^{\prime}$ is the residual computed for the trial location. Equation (3) is expressed in matrix form:

$$
G m=d \text {, }
$$

where $G$ is the operator matrix acting on the unknown column model matrix $m=[\delta t, \delta \phi, \delta \lambda, \delta z]^{T}$, $\mathrm{d}$ is the data matrix of travel time residuals. An estimate of $m$ is obtained by: 


$$
m=[G+\beta I]^{-1} d,
$$

where $I$ is the identity matrix to dampen the solution (Menke, 1984, $\sec 4.7 .3$ ), and $\beta$ is the damping factor. The necessary adjustment vector is solved by requiring

$$
\sum_{i} w_{i} \varepsilon_{i}^{2}=\text { a minimum, }
$$

where $w_{i}$ is the weight, and the sum is over $i$ observations. Applying Eq. (6) to Eq. (3) leads to four equations and the four unknowns of the adjustment vector:

$$
\begin{aligned}
& \frac{\partial}{\partial \phi} \sum \frac{w_{i} \varepsilon_{i}^{2}}{\sigma_{i}^{p}}=-\sum \frac{w_{i} a_{i} R_{i}^{\prime}}{\sigma_{i}^{p}}+\sum \frac{w_{i} a_{i}}{\sigma_{i}^{p}} \delta t+\sum \frac{w_{i} a_{i}^{2}}{\sigma_{i}^{p}} \delta \phi+\sum \frac{w_{i} a_{i} b_{i}}{\sigma_{i}^{p}} \delta \lambda+\sum \frac{w_{i} a_{i} c_{i}}{\sigma_{i}^{p}} \delta z=0 \\
& \frac{\partial}{\partial \lambda} \sum \frac{w_{i} \varepsilon_{i}^{2}}{\sigma_{i}^{p}}=-\sum \frac{w_{i} b_{i} R_{i}^{\prime}}{\sigma_{i}^{p}}+\sum \frac{w_{i} b_{i}}{\sigma_{i}^{p}} \delta t+\sum \frac{w_{i} a_{i} b_{i}}{\sigma_{i}^{p}} \delta \phi+\sum \frac{w_{i} b_{i}^{2}}{\sigma_{i}^{p}} \delta \lambda+\sum \frac{w_{i} b_{i} c_{i}}{\sigma_{i}^{p}} \delta z=0 \\
& \frac{\partial}{\partial z} \sum \frac{w_{i} \varepsilon_{i}^{2}}{\sigma_{i}^{p}}=-\sum \frac{w_{i} c_{i} R_{i}^{\prime}}{\sigma_{i}^{p}}+\sum \frac{w_{i} c_{i}}{\sigma_{i}^{p}} \delta t+\sum \frac{w_{i} a_{i} c_{i}}{\sigma_{i}^{p}} \delta \phi+\sum \frac{w_{i} b_{i} c_{i}}{\sigma_{i}^{p}} \delta \lambda+\sum \frac{w_{i} c_{i}^{2}}{\sigma_{i}^{p}} \delta z=0 \\
& \frac{\partial}{\partial t} \sum \frac{w_{i} \varepsilon_{i}^{2}}{\sigma_{i}^{p}}=-\sum \frac{w_{i} R_{i}^{\prime}}{\sigma_{i}^{p}}+\sum \frac{w_{i} N}{\sigma_{i}^{p}} \delta t+\sum \frac{w_{i} a_{i}}{\sigma_{i}^{p}} \delta \phi+\sum \frac{w_{i} b_{i}}{\sigma_{i}^{p}} \delta \lambda+\sum \frac{w_{i} c_{i}}{\sigma_{i}^{p}} \delta z=0 .
\end{aligned}
$$

The constants:

$$
a_{i}=\frac{\partial t_{i}}{\partial \phi} ; b_{i}=\frac{\partial t_{i}}{\partial \lambda} ; c_{i}=\frac{\partial t_{i}}{\partial z}
$$

$N$ is the number of observations, and $\sigma_{i}^{p}$ is the prior variance of the $i^{\text {th }}$ observation and normalizes the system of equations for mixed-mode observations so that the residual for each data point is compared to its expected error (Jackson, 1972). Prior observations are used to estimate variance [outlined for Eq. (30) below] because with sparse data the variance estimate based on observations for any one earthquake are insufficient to obtain a good estimate of variance. Normalizing by variance is weighting the location estimate by the more reliable phases. This is different than the weights $w_{i}$ applied, which are an estimate of the quality of a particular arrival.

If the trial location is too far from the actual location, the Taylor series is not adequate, and the solution is attempted again starting from the new location. This process is repeated until the adjustment vector reaches some minimum value.

Four observations are required for a solution; three are required if depth is held fixed. Multiple observations provide a redundancy that averages out random errors in arrival times. With weighted least squares, the sum of the number of phases times their weight must equal four or three, respectively.

Range estimates based on relative arrival times are automatically included in the inversion since all identifiable phases are used. Consider $\tau_{i}$ to be the relative arrival time of two phases, then

$$
\dot{t}_{i}\left(\phi, \lambda, z, t, \phi_{s}, \lambda_{s}\right)=\Delta\left(\frac{1}{V_{2}}-\frac{1}{V_{1}}\right)+c_{2}-c_{1}
$$


where $c_{1}$ and $c_{2}$ are delay times (zero for direct arrivals), and $V$ is velocity. Similarly, range estimates based on arrival times at two stations are:

$$
\dot{t}_{i}\left(\phi, \lambda, z, t, \phi_{s 1}, \lambda_{s 1}, \phi_{s 2}, \lambda_{s 2}\right)=\frac{\Delta_{2}}{V_{2}}-\frac{\Delta_{2}}{V_{2}}+c_{2}-c_{1},
$$

both of which satisfy the linear combination of observed arrival times.

Mixed-mode solutions require expressing $t_{i}^{\prime}\left(\phi, \lambda, z, t, \phi_{s}, \lambda_{s}\right)$ for the different observations and taking the partial derivatives to obtain the constants. However, this alone will improperly weight the observations. For example, backazimuth measurements will have a contribution to Eq. (3) expressed as:

$$
R_{i}^{\prime}=w_{i}\left(B A Z_{o b s}-B A Z_{c a l}\right) \text {. }
$$

However, consider the error in location from the backazimuth estimate to be the distance $\Delta_{i}$ from a point along the observed backazimuth direction; the same distance from the station to the trial location. Then, the error in terms of travel time is:

$$
R_{i}^{\prime}=w_{i} \frac{\Delta_{i}\left(B A Z_{o b s}-B A Z_{c a l}\right)}{V_{i}} .
$$

The small angle approximation for $\sin \left(B A Z_{o b s}-B A Z_{c a l}\right)$ is applied. Then a residual based on backazimuths only is improperly weighted by $V_{i} / \Delta_{i}$.

The partial derivatives and backazimuth are calculated for a geocentric earth model (Herrmann, 1982) with the relations for distance:

$$
\cos \Delta=\cos \phi_{e} \cos \phi_{s} \cos \left(\lambda_{e}-\lambda_{s}\right)+\sin \phi_{e} \sin \phi_{s}
$$

and relations for backazimuth are:

$$
\begin{aligned}
& \sin B A Z=\frac{-\cos \phi_{e} \sin \left(\lambda_{s}-\lambda_{e}\right)}{\sin \Delta}, \\
& \cos B A Z=\frac{\sin \phi_{e}-\cos \Delta \sin \phi_{s}}{\sin \Delta \cos \phi_{s}} .
\end{aligned}
$$

There are several conditions on the backazimuth relations if they are used separately, but

$$
B A Z=\operatorname{atan}\left(\frac{\sin B A Z}{\cos B A Z}\right)
$$

has no restrictions if the sign of the numerator and denominator are preserved, as in the $\operatorname{atan} 2(\sin B A Z, \cos B A Z)$ FORTRAN function.

In the above relations, $\phi$ and $\lambda$ are geocentric latitude and longitude, respectively, and $\Delta$ is distance in degrees $\left(1^{\circ}=111.12 \mathrm{~km}\right)$. The subscript $e$ refers to event location, and the subscript $s$ refers to station location.

Geographic latitude and longitude $\left(\phi^{\prime}, \lambda^{\prime}\right)$ are defined with the convention:

$-90^{\circ} \leq \phi^{\prime} \leq 90^{\circ}$, positive for north,

and

$-180^{\circ}<\lambda^{\prime} \leq 180^{\circ}$, positive for east 
Geocentric latitude and longitude are obtained from the relations:

$$
\begin{aligned}
\tan \phi & =(1-\gamma)^{2} \tan \phi^{\prime}=0.993277 \tan \phi^{\prime}, \\
\lambda & =\lambda^{\prime},
\end{aligned}
$$

where $\gamma$ is the correction factor for earth ellipticity $(\gamma=0.003367003)$.

The partial derivatives of travel times are

$$
\frac{\partial t_{i}}{\partial \phi_{e}}=\frac{\sin \phi_{e} \cos \phi_{s} \cos \left(\lambda_{e}-\lambda_{s}\right)-\cos \phi_{e} \sin \phi_{s}}{V_{k} \sin \Delta}
$$

and

$$
\frac{\partial t_{i}}{\partial \lambda_{e}}=\frac{\cos \phi_{e} \cos \phi_{s} \sin \left(\lambda_{e}-\lambda_{s}\right)}{V_{k} \sin \Delta}
$$

Units are s/deg, which are controlled by units of $V_{k}$. For backazimuth estimates,

$$
\frac{\partial B A Z_{i}}{\partial \phi_{e}}=\frac{-\cos \phi_{e}}{\sin B A Z \cos \phi_{s} \sin \Delta}
$$

and

$$
\frac{\partial B A Z_{i}}{\partial \lambda_{e}}=\frac{\cos \left(\lambda_{e}-\lambda_{s}\right) \cos \phi_{e}}{\cos B A Z \sin \Delta},
$$

where $B A Z_{i}$ distinguishes backazimuth observations previously identified only as $t_{i}$. These partials are unitless (deg/deg). Note that the relations:

$$
\frac{\partial \cos \theta}{\partial \chi}=\sin \theta \frac{\partial \theta}{\partial \chi} ; \frac{\partial \sin \theta}{\partial \chi}=\cos \theta \frac{\partial \theta}{\partial \chi}
$$

make the partial derivatives simple to compute.

The algebraic solution from one station where backazimuth and distance are known is:

$$
\begin{aligned}
& \phi_{e}=\sin ^{-1}\left[\sin \phi_{s} \cos \Delta+\cos \phi_{s} \sin \Delta \cos B A Z\right] \\
& \lambda_{e}=\lambda_{s} \pm \cos ^{-1}\left[\frac{\cos \Delta-\sin \phi_{s} \sin \phi_{e}}{\cos \phi_{s} \cos \phi_{e}}\right],
\end{aligned}
$$

where the second term on the right is added if $B A Z$ is $\leq 180.0^{\circ}$, and it is subtracted if $B A Z$ is $>180.0^{\circ}$. 


\section{Error Estimate}

Standard means by which the location error is estimated for least-squares problems require some modification when mixed-mode and sparse dain are used. The errors in location are assumed to be a result of random errors from misidentification of phase arrival times and heterogeneities in the velocity structure and the result of systematic errors from incorrec ly estimating the vclocity model. In the following explanation, the conventional method for estimating location errors resulting from random errors in location algorithms is outlined, and then the rethod for estimating location errors using sparse data is discussed. Systematic errors are addressed later. The variance is an indication of the random error and is generally obtained by:

$$
\sigma_{d}^{2}=\frac{1}{N-L} \sum_{i=1}^{N} w_{i} R_{i}^{2},
$$

for $N$ observations and $L$ model parameters. The subscript $d$ refers to the estimate from the data used in the location only; $\sigma_{d}^{2}$ is the data variance. Subscripts are used here for $d$ and $p$ instead of superscripts for visual clarity. If the variance is computed from prior observations, as discussed below, then Eq. (25) represents $\sigma_{p}^{2}$

The covariance matrix is generally used to estimate crrors in model parameters. The covariance matrix is:

$$
V=\sigma_{d}^{2}\left[G^{T} G\right]^{-1}
$$

and the diagonals are variance estimates for model parameters.

The joint confidence ellipsoid for model parameters is defined by:

$$
(m-\bar{m})^{-1} V^{-1}(m-\bar{m})=c_{L}^{2}
$$

where $\bar{m}$ is the unknown true model parameter, and $c_{L}$ depends on the confidence coefficient:

$$
c_{L}^{2}=L \sigma_{d}^{2} F(p ; L, N-L),
$$

where $F(p ; L, N-L)$ is the $F$ statistic with $L$ and $N-L$ degrees of freedom at the $100 \%$ confidence level, and $p$ is the confidence probability sought (Flinn, 1965).

This approach is adequate when an event is located by a large number of arrivals so that $\sigma_{d}^{2}$ is a reasonable estimate of the true variance, but for sparse data this is not generally true. Jordan and Sverdrup (1981) have addressed this problem, and their approach is used here. They suggest adnpting the Baysian technique of imposing a prior distribution based on previous recordings of phase travel times to estimate the true variance:

$$
\sigma^{2}=\frac{(K-L) \sigma_{p}^{2}+(N-L) \sigma_{d}^{2}}{K+N-L}
$$

for $K$ and $N$ prior and data observations, respectively.

In the situation studied here where multiple phases and backazimuths are used, each phase may have a different variance for each station and velocity model used. In RECLOC, prior variance is only 
computed from the phases and stations used in the location. The prior variance is computed for good arrivals only, and all weights are assigned 1.0, except when obtaining prior variance for backazimuth, as discussed below.

Although the prior error in backazimuth may be estimated, the effect on earthquake location is distance dependent. Therefore, the sum of residuals for a particular station from backazimuth is converted to seconds by:

$$
R_{i}^{2}=\sum_{K} \frac{\Delta_{i}^{2}}{V^{2}} w_{i} R_{i}^{2},
$$

where $\Delta_{i}$ is the distance from the station to the event location. Backazimuth estimates are so unreliable that they are only used in locations with three or fewer stations. When locating with two or three stations, the backazimuth estimates are first weighted to 0.1 , then distance weighted so that $w_{i}=(0.1 * 200 \mathrm{~km}) / \Delta_{i}$. Backazimuth residuals are not very reliable for computing error ellipses either. For example, the rootmean-square contribution, without weighting, from an arrival $2000 \mathrm{~km}$ distant with a BAZ prior error of $20^{\circ}$ would be $196 \mathrm{~s}$. This leads to unrealistically large error ellipses. Therefore, errors to backazimuth are only used to contribute to a prior variance when locating with a single station, and then an ad hoc weighting of 0.1 is applied to bring error ellipses to a size that experimentally fit the $95 \%$ confidence requirement.

The $G$ matrix was previously normalized for the mixed-mode problem by dividing the contribution from each observation by $\sigma^{p}$ to obtain units of $1 / \mathrm{deg}$. Now, the $G$ matrix is multiplied by $\sigma$ to arrive at $\mathrm{s}^{2} / \mathrm{deg}$ units for computations of the error ellipse. The joint confidence location error ellipse is determined from $E$, the second order covariance matrix of horizontal location parameters $(\phi, \lambda)$, and the variance $\sigma$. Then, from Eqs. (27) and (28):

$$
c_{2}^{2}=l_{1} \Delta M_{\phi}^{2}+l_{2} \Delta M_{\lambda}^{2}
$$

with

$$
\begin{aligned}
& c_{2}^{2}=2 F(0.95 ; 2, K+N-2) \sigma \\
& l_{1}=E_{11}^{-1} \cos ^{2} \alpha+2 E_{12}^{-1} \sin \alpha \cos \alpha+E_{22}^{-1} \sin ^{2} \alpha \\
& l_{2}=E_{11}^{-1} \sin ^{2} \alpha-2 E_{12}^{-1} \sin \alpha \cos \alpha+E_{22}^{-1} \cos ^{2} \alpha .
\end{aligned}
$$

$F$ is obtained from tables (see CRC, Standard Math Tables, p. 272), where for example $F(0.95 ; 2,15)=3.68$. $\alpha$ is the angle from north for the $\Gamma_{1}$ axis:

$$
\alpha=0.5 \tan ^{-1}\left[\frac{2 E_{12}^{-1}}{E_{11}^{-1}-E_{22}^{-1}}\right] .
$$


The length of the semiaxes are:

$$
\Gamma_{1}=111.12\left(\frac{c_{2}^{2}}{l_{1}}\right)^{1 / 2},
$$

and

$$
\Gamma_{2}=111.12\left(\frac{c_{2}^{2}}{l_{2}}\right)^{1 / 2} .
$$

$\Gamma_{1}, \Gamma_{2}$ are multiplied by $111.12 \mathrm{~km} / \mathrm{deg}$ to obtain the length of possible location error (in kilometers) for an epicenter at the center of the ellipse; these with $\alpha$ are output in REGLOC. REGLOC also provides the $95 \%$ limits of the model parameters, assuming a Gaussian distribution:

$$
\delta m=\sqrt{-2 \sigma^{2} \ln \left[P(m)(2 \pi)^{1 / 2} \sigma\right]}
$$

in units of degrees.

\section{RSTN Location Capability}

The data used for evaluating the location capability of seismic recordings made by the RSTN consist of records of 94 earthquakes identified by local network bulletins and recorded on the RSTN (Jarpe et al., 1986); epicenters are plotted in Figure 2 and are listed in Table 2. The events used in this analysis were randomly selected, but an even distribution in location and magnitude was attempted.

The spatial limits of events considered in this study are the western boundary of the Rocky Mountains, the East Coast, the northern extent of the Canadian land mass, and the southern extent of the United States. This generally encompasses the Canadian Shield and is approximately equal to the land mass of the former Soviet Union. Figure 2 shows that many of the events are located outside the perimeter of the network. In addition, there is an uneven distribution of stations over the region. The eastern portion of Canada has poor station coverage, and the central portion of the United States has the highest density of stations.

Phase detection and identification are done in a manner that optimizes the number of phases used. Time series files are bandpassed in four frequency bands $(0.5-1,1-2,2-4$, and $4-8 \mathrm{~Hz})$ and then are plotted. The expected arrival time of the phases are marked on the records (based on the known location of the event), and the arrival times are picked by eye. Identifying the phases from prior knowledge is justified under the assumption that phase identification will be possible with signal processing and artificial intelligence programs (Jarpe et al., 1991). Location analysis is then performed with the best data that can be obtained. Phases are assigned a weight based on the signal-to-noise ratio as outlined in Table 3. Backazimuths are obtained from the MUSIC algorithm (Dowla and Harris, 1987). Appendix A lists the phase arrival times and backazimuths read for the 94 events.

All events with at least a minimum amount of information are located. Depths are constrained to $10 \mathrm{~km}$, since depth cannot be resolved with arrivals at regional distances. Therefore, there are 3-degrees -of freedom in the locations. Table 4 lists the minimum information necessary to obtain an epicentral location for one, two, or three stations. Each column represents a combination of arrival times from any identifiable arrival (AT) and backazimuths (BAZ) that can be obtained at a single station. An $X$ is placed on the contribution from a single station that is necessary for a location with the number of stations listed in 
column one. More than one backazimuth at one station is averaged to obtain a single value, and more than two arrival times at a single station are used to obtain an average distance estimate. Redundant information is not listed. For example, two arrival times and a backazimuth at one station are sufficient for a location, and this is not listed as a combination for two or three stations. At two stations, one arrival time and backazimuth at one station and a backazimuth at another are redundant to two backazimuths.

The location algorithm is used even if only the minimum number of phases or backazimuths is available, except for locating with one station, in which case an algebraic solution is obtained. Other locations are nonlinear in $\phi$ and $\lambda$ through sines and cosines, and a solution with linear algebra is not possible.

Table 5 shows the number of events in each magnitude range, and the events that had at least the minimum information required for a location.

\section{Location Accuracy with the Average Crustal Model}

The average velocity model for each shield region used is listed in Table 6. Prior estimates of travel time variance are obtained by locating all of the events and by using the residuals obtained as input into Eq. (25). The location error estimates are then obtained by calculating the locations again. Table 7 shows the individual phase-travel-time residual squared and the number of readings for each phase at each station used for the error analysis. To avoid biasing the prior variance values, only residuals from locations using more than the minimum data required are used. Also, only residual values from phases that were used in the final solution are used. Residual squared values shown for backazimuth estimates are in degrees.

Table 8 is a summary of RSTN location information, the location error as compared to published reports, and to the maximum semi-axis of the error ellipse estimated by REGLOC. Ten of the ninety.four events had a location error greater than the maximum of the semi-axis of the error ellipse, and these ten event location errors were generally close to the predicted. The relation of the error ellipses to published locations is discussed later. Large location errors primarily occur when the locations were determined using information from one or two stations that rely on backazimuth estimates in the computations. Single station locations in general have the worst location error; the average location error for these events is 453 $\mathrm{km}$. This is not surprising considering the nearly $20^{\circ}$ un-reliability of backazimuth estimates. Two station locations are generally considerably better than single station locations since the backazimuth estimates are not primary to the location estimate but do help in distinguishing between dual solutions that result if only travel times are used. Locations based on two stations have an average location error of $85.0 \mathrm{~km}$. The average location error for the events located with three or more stations is $73.4 \mathrm{~km}$. For purposes of test ban treaty verification, single station locations have extreme limitations, but two station locations can reliably estimate the general location of an event.

Figure 3 is a plot of the location error as a function of magnitude. Most of the events with a magnitude $<3.0$ are located with one station and have the largest location error. Events with a magnitude 3.0 and greater have an average location error of $61 \mathrm{~km}$ (excluding the three outliers) and the location error is fairly independent of magnitude. Nineteen of the 38 events with magnitudes 3.0-3.6 are located with two stations, and 18 of the 20 events above magnitude 3.6 are located with three or more stations.

Figure 4 shows $95 \%$ confidence error ellipses for events using two stations to estimate their location and the actual location; Figure 5 shows error ellipses for events using three or more stations to estimate their location. Even though the absolute location errors are predicted by the error ellipses, some of the actual locations of the events are outside the error ellipses. This is a result of a systematic error in 
location resulting from an incorrect velocity model. Nine of the 26 events located with two stations and 20 of the 35 events located with three or more stations have locations outside the $95 \%$ confidence error ellipses. Figures 6 and 7 show $99 \%$ confidence error ellipses for the same events shown in Figures 4 and 5 . Here, 5 and 9 events, respectively, fall outside the erro: ellipses. Examination of Figures 6 and 7 show that events that do not have locations within the error ellipses are very close to the error ellipses. Error ellipse accuracy is discussed in more detail later.

In summary, events with a magnitude $<3.0$ are principally located with one station and have extremely unreliable locations. Events with magnitude 3.0 and greater are principally located with two or more stations and have an average location accuracy of about $61 \mathrm{~km}$. Ninety-five percent confidence limits are systematically displaced because of the inadequacy of the average crustal velocity model, and $99 \%$ error ellipses are a better estimate of the location for events using an average crustal model.

\section{Location Accuracy with Regional Velocity Models and Station Corrections}

Travel times from well-located large events and additional information about the regional geology can be used to further improve the location accuracy. Figure 8 shows the study area divided into four regions (two of the regions are further subdivided for station corrections) to allow a more detailed study of the location capability of the RSTN data. Events that have an initial location in one of the four regions are relocated with a velocity model for that region along with station corrections appropriate for events from that region. A master event located within each region, or subregion, is used to obtain travel time corrections to all stations. All events within the region are then relocaied with the station's travel time corrections appropriate for the region.

Figure 1 shows the major crustal provinces for North America from Taylor and Qualheim (1983). Velocity models for the Churchill, Superior, Central, and Grenville provinces are listed in Table 9; they are described by the thickness of the crust above and below the Conrad discontinuity, the corresponding average velocity, and the velocity of the upper mantle. The Churchill province velocity model is obtained from the refraction studies of Prodehl (1970) and the Rayleigh wave dispersion of Keller et al. (1976). Both studies agree fairly well on the main features of the velocity structure. The structures for the Superior and Grenville provinces are obtained from the refraction studies of Berry and Fuchs (1973) and the receiver function studies of Owens et al. (1987). The structure for the Central province is obtained from Carts and Bollinger (1981). They obtain velocities of $P_{g}, P_{n}, S_{n}$, and $L_{g}$ by plotting arrivals from a well-located $M_{L}=$ 3.7 earthquake. They argue that the Conrad discontinuity is not well-defined or nonexistent in this region, and they develop a layer over the half-space velocity model. Even so, it is difficult to accept a continuous velocity for a $39 \mathrm{~km}$ increase in depth; therefore, an intermediate layer is included for this study.

Master events used to obtain travel time corrections for events are indicated by an asterisk in Table 2 and are plotted with a different symbol in Figure 8. Station corrections are obtained by locating events with the regional velocity model (without station corrections), and with a priori travel time residuals for the average earth model listed in Table 7. Table 10 lists the travel time residuals obtained from the master events that are used to obtain station corrections. No master event is used for the Churchill I area, because there were no well-recorded events. The location error between local network and REGLOC master event locations was small for all regions except for the Superior and Churchill III provinces master events, which had a location errors of 50 and $25 \mathrm{~km}$, respectively. These events, and the resulting station corrections, were used anyway because in a situation where the actual earthquake location was not known, they would have been used.

To obtain Baysian statistics for the estımation of error ellipses, all events are first located with the appropriate velocity model and station corrections, but a priori travel time residuals for the average earth model listed in Table 7 are used to calculate $\sigma_{p}$ to normalize the location cquations [Eq. (25)]. Once all the 
events for a particular province or subprovince are located, then a priori travel time residuals particular for that region are calculated, these are listed in Table 11. Only phases with at least 3 residuals are used to calculate a priori values since the same residuals. will be included again in the final runs and will effectively be used twice (although the final runs have different locations, and residuals, when the a priori values are used to calculate variance for inversion are changed). The master events are not used to calculate a priori values since their residuals are forced to be zero by the selection of the station corrections. Phases with zeros in Table 11 did not have enough phases recorded to get a priori values; those from the average model in Table 7 were used instead for location calculations. The events are then located again using the $a$ priori statistics. Locations change slightly because of the different statistics used for normalization. The a priori residuals calculated for the Churchill II area are also used for the Churchill I province. Final locations and location error information are listed in Table 12.

Single station locations remain extremely unreliable, as they are when obtained with the average crustal model. The average location error for single station locations improved, but was $414 \mathrm{~km}$. Locations with two stations improved, and the average location error was $68.3 \mathrm{~km}$. For events located with three or more stations, and the average location error also improved and was $34.8 \mathrm{~km}$. Events used as master events are included in computing average location errors since their locations were not fixed by the local network locations, and were determined in the same manner as other events. Location errors using regional velocity models and station corrections provides $20 \%$ and $53 \%$ improvement in location accuracy over the average velocity models for the 2 station and 3 or greater station locations, respectively. Figure 9 shows location error as a function of magnitude. For events with a magnitude of 3.0 and greater, the average location error is 55.3 and is fairly independent of magnitude.

Figures 10 and 11 show $95 \%$ confidence error ellipses for events using two stations and three or more stations, respectively, along with the actual location of the event. The error ellipses are actually a little larger than those obtained from the average crustal model; compare the last columns in Tables 8 and 12. The error ellipses are larger because the random errors have slightly increased with the use of the calibrated crustal models. However, the systematic errors have decreased. This is evident by the number of error ellipses that now include the actual locations of the events: 48 of the 59 events have error ellipses that include the actual locations of the events, as compared to 33 of the 59 events when the average crustal model is used. The fact that fewer than $95 \%$ of the events in either case do not fall within the $95 \%$ confidence ellipses is possibly because error ellipses only take into account random errors and the systematic errors have not all been accounted for. When only events located with three or more stations are used 28 of 33 events ( $85 \%$ ) have locations within the $95 \%$ error ellipses (Figure 11), and indicate a reliability of error ellipse estimation for these events; only 16 of 33 the events fall within the $95 \%$ error ellipse for the average crustal model (Figure 5). Figures 12 and 13 show $99 \%$ confidence error ellipses for the same events shown in Figures 10 and 11, where 51 of the 59 events have error ellipses that include the actual locations of the events, as compared to 44 of the 59 events when the average crustal model is used. When only events located with three or more stations are used 30 of 33 events $(90 \%)$ have locations within the $99 \%$ error ellipses (Figure 11), and indic e a reliability of error ellipse estimation for these events.

In summary, only events located with three or more stations, and using regional velocity models and master events have location error ellipse that are reliable, but only at the $85 \%$ and $90 \%$ level when $95 \%$ and $99 \%$ error ellipses are used, respectively. For events located with two stations only $77 \%$ of the events are located within the $95 \%$ confidence error ellipses, and statistics are not significantly improved by using $99 \%$ error ellipses. Similarly, for events located with the average crustal model only $65 \%$ and $48 \%$ of the events fall within the $95 \%$ confidence ellipses for the two and three or more station locations, respectively. 


\section{Percentage of Events Located}

For purposes of test ban treaty verification, the percentage of events located refers to events that can occur anywhere within the network. This assumes an even distribution of events throughout the network. This is distinctive from percentage of actual events occurring within the network, which is biased by the distribution of seismic sources and differing seismicity rates.

Since the 94 events studied here are not evenly distributed in magnitude and location, a straightforward analysis will not yield a reliable assessment of the percentage of events that could be located. Rather, an analysis of detection capability along with location capability is used here for the assessment.

Jarpe et al. (1986) assessed the detection capabilities of RSTN data using the same data set as used for this study along with an additional 116 events. The time series were bandpassed and expected arrival times were marked on data files as described above. They then measured the maximum trace amplitudes on all three components near the time expected for $P_{n}, P_{g^{\prime}}, S_{n}$ and $L_{g}$. They calculated the signal-to-noise ratio (SNR) for each phase in each band on each component by dividing the maximum phase window amplitudes by the pre-event noise amplitude. A phase detection occurred when the SNR in the velocity window exceeded a threshold of 3.0. They found that 3.0 was the lowest value that provided real phase detections as opposed to spurious detections (based on a visual inspection of amplitude); they did not examine a false alarm rate. An SNR of 3:1 is a stiffer criteria than is necessary to obtain information that can be used for a location, but is a good minimum SNR for ensuring good data, a minimum number of spurious arrivals, and good quality backazimuths. This then is accepted as a good criteria for assessing location capability.

Figure 14 (from Jarpe et. al., 1986) shows the percentage of phases (of 848 possible) detected versus magnitude and recording distance. This is irrespective of source region and recording station. This, then represents an average value for the network and does not account for regional differences in attenuation. Considering that the longest midpoint distance between any two stations of the RSTN is about $1200 \mathrm{~km}$ (the northern extreme of eastern Canada is greater), the point along the curves in Figure 14 at $11^{\circ}$ represents the minimum number of phases detected from events anywhere within the network. For example, a minimum of $40 \%$ of the phases for magnitude $M=2.5-3.0$ and $80 \%$ of the phases for magnitude $M=3.0-3.5$ should be recorded. Since we only need 2 phases to obtain a location (Table 4 ), $100 \%$ of the events greater than magnitude 3.0 and slightly less $100 \%$ of the total number of events greater than magnitude 2.5 should have been located. The results of this study indicate fewer than expected percentage of events were actually located; as is evident from Table 5, where $89 \%$ of the events with magnitude greater than 2.5 were actually located. Regional variations in recording capability and random or systematic interruptions in recording can also affect the results.

Figure 15 shows the Jarpe et al. (1986) analysis of percentage of detections versus recording distance, irrespective of magnitude, for each station. Considering a fairly even distribution of sources for each station, this indicates the variation of recording capability resulting from regional differences in geologic structure. Figure 1 shows the major geologic-seismic velocity provinces of the RSTN region (from Owens et al., 1987). Station RSCP and RSNY, for example, record arrivals traversing the Grenville Orogene belt, which has a lower $Q$ factor than the interior U.S. and Canadian shields, and a significantly lower percentage of arrivals are recorded. The relative percentage of events located by each station versus distance is taken as the effect of the regional geologic structural differences. For example, the average percentage detected at $1000 \mathrm{~km}$ is $75 \%$, and station RSNT detects $50 \%$. It then has a $33 \%$ depletion in recording because of regional differences. Since the curves in Figure 15 are fairly parallel, this suggests that the effect is fairly uniform for all distances. To incorporate regional differences in Figure 14, the curves are 
multiplied by the depletion or amplification factor, from the average, to represent that from an individual station. Figure 14 modified for station RSTN, for example, would indicate that $34 \%$ of the phases for magnitude $2.5-3.0$ and $50 \%$ of phases for magnitude $3.0-3.5$ would be recorded at $1200 \mathrm{~km}$. Considering these factors for all stations it is estimated that about $90 \%$ of the events with magnitude greater than or equal to 2.5 and $100 \%$ of events greater than or equal to 3.0 should have been located. Since, as discussed previously, fewer than $100 \%$ of the events greater than or equal to magnitude 2.5 (Table 5) were actually located, it is concluded that this is a result of spurious interruptions in data recording or anomalously attenuating travel paths. Figure 16 shows the estimation of the number of events that should be located as a function of distance. This is obtained by modifying table 14 for the differences in regional recording capability as described, and integrating over all recording distances. Figure 16 also shows the results from Table 5. The differences between the two curves is possibly due to spurious interruptions in recording.

\section{Discussion and Conclusions}

It is rather surprising that with such a sparse network of the five three-component stations covering most of North America that most events with magnitude greater than 2.5 can be located, and with a fairly reliable accuracy. It was found that approximately $90 \%$ of all magnitude $\geq 2.5$ events occurring anywhere within the RSTN can be detected and located and that approximately $93 \%$ of events with a magnitude $\geq 3.0$ can be detected and located. Further, tests of phase recordings as a function of magnitude and distance suggest that the RSTN should detect and locate $100 \%$ of the events with a magnitude $M \geq 3.0$. This discrepancy is attributed to random interference and interruptions in data collection and to the variability in the quality of the arrivals. Even so, some phases used in this study were generated by events with magnitudes $<3.0$ that were recorded over $3000 \mathrm{~km}$ from where they occurred, yet the data still provided enough information to locate the event within $50 \mathrm{~km}$. Much of this capability is attributed to recording the $L_{g}$ phases, which in a shield region channels the $S$-wave energy and has very low attenuation. All phases are generally recorded for events with magnitudes $>3.5$. Forty-two percent of all phases recorded are $L_{8}$ phases; the $S_{n}$ phase is the least useful with only $10 \%$ recorded for distarices $>200$ $\mathrm{km}$. The implications are obvious-this study would have significantly different results if we were examining a region that provided poor transmission of the $L_{g}$ phase.

Most events with a magnitude $<3.0$ can only be located with arrivals from one station. Their average location errors are 453 and $414 \mathrm{~km}$ for the average- and regional-velocity model locations, respectively. Single station locations are very unreliable because they depend on accurate backazimuth estimates, and backazimuth proved to be a very unreliable computation. This is partially due to the fact that only small events required a single station location and that these also had a very poor SNR. However, even events with high SNRs have a backazimuth error of $\pm 15^{\circ}$. Considering that the location error linearly increases with distance when locating with backazimuth estimates, this too suggests that backazimuth is a very poor measurement to use.

Fifty percent of the events with magnitudes of 3.0-3.5 are located with two stations. The average location errors when locating with two stations are 85 and $68 \mathrm{~km}$ for the average- and regional-velocity model locations, respectively. Locations with two stations are almost as accurate as locations with three or 
more stations; this is because backazimuth is not primary to the location computation, but only acts to distinguish between ambiguous interpretations.

Ninety percent of the events above a magnitude of 3.5 are located with three or more stations. Their average location errors are 73 and $35 \mathrm{~km}$, for the average- and regional-velocity model locations, respectively. The average location accuracy for events above a magnitude of 3.5 does not depend on magnitude. Error ellipse estimates only account for the random errors in location. Fifty-six percent of the events located with the average crustal model and $82 \%$ of the events located with regional velocity models have locations that fall within the $95 \%$ confidence error ellipses. This suggests that including regional velocity models and calibrated travel times from master events does not account for all of the systematic errors. Most error ellipses that do not include the actual location of events are close to the event. Seventyfive and $86 \%$ of events fall within the $99 \%$ confidence error ellipses for the average and regional crustal models, respectively. For purposes of test ban treaty verification, then, it is recommended that $99 \%$ error ellipses be calculated, but only considered to be reliable at the 75 and $86 \%$ confidence level. For purposes of test ban treaty verification it is concluded that $100 \%$ of events above magnitude 3.0 could be detected and located with accuracy less than $68 \mathrm{~km}$, and $99 \%$ error ellipses calculated are reliable at the $86 \%$ confidence level. Events below magnitude 3.0 cannot be fully detected and their location accuracy is fairly poor.

\section{References}

Bratt, S. R., and T. C. Bache (1988), “Locating Events with a Sparse Network of Regional Arrays,” Bull. Seismol. Soc. Am. 78, 780-798.

Berry, M. J., and K. Fuchs (1973), "Crustal Structure of the Superior and Grenville Provinces of the Northeastern Canadian Shield," Bull. Seismol. Soc. Am. 63, 1939-1432.

Carts, D. A., and G. A. Bollinger (1981), "A Regional Crustal Model for the Southeastern United States,"

Bull. Seismol. Soc. Am. 63, 1939-1432.

Dowla, F.U. and D.B. Harris (1987) Direction estimation of vector planewave fields, Proc. IEEE International Conference on Acoustics Speech and Signal Processing 2225-2228.

Flinn, E. A. (1965), "Confidence Regions and Error Determinations for Seismic Event Location," Rev. Geophy. 3(1), 157-185.

Foy, W. H. (1976), "Position-Location Solutions by Taylor-Series Estimation," IEEE Trans. on Aerosp. and Elect. Sys. AES-12, 187-193.

Herrmann, R. B. (1982), “Digital Processing of Regional Network Data," Bull. Seismol. Soc. Am. 72, part B, S261-S276.

Jarpe, Stephen P., Carolyn Baily, Brenda C. Bowman, Elizabeth K. Foote, Keith J. MacKay, Robert M. Searfus, Joseph E. Tull (1991) Deployable Seismic Verification System (DSVS): System Evaluation Center (SEC) Software Documentation. Version 1.0, Lawrence Livermore National Laboratory, M281

Geiger (1910), apparently the first person with the idea of minimizing a sum of weighted squared residuals to estimate hypocentral coordinates.

Jackson, D. D. (1972), "Interpretation of Inaccurate, Insufficient and Inconsistent Data," Geophys. J. R. Astr. Soc. 28, 97-109. 
Jarpe, S. P., T. F. Hauk, and K. K. Nakanishi (1986), "Detection of Regional Earthquakes in Eastern North America using the RSTN in the Frequency Range 0.5 to $16 \mathrm{~Hz}$," Proceedings of the $1986 \mathrm{RSTN} /$ NORESS Research Symposium, Las Vegas, Nevada April 30-May 1, 1986, Lawrence Livermore National Laboratory, Livermore, CA, Conf-8604196.

Jordan, T. H., and K. A. Sverdrup (1981), "Teleseismic Location Techniques and their Applications to Earthquake Clusters in the South-Central Pacific," Bull. Seismol. Soc. Am. 71, 1105-1130.

Keller, G. R., R. B. Smith, L. W. Braile, R. Heaney, and D. H. Shurbet (1976), “Upper Crustal Structure of the Eastern Basin and Range, Northern Colorado Plateau, and Middle Rocky Mountains from Rayleigh-Wave Dispersion," Bull. Seismol. Soc. Am. 66, 869-876.

Lee, W. and J. Lahr (1971) HYPO71, a hypocentral location program; U.S. Geological Survey.

Menke, 'N. (1984), Geophysical Data Analysis: Discrete Inverse Theory, Harcourt Brace Jovanovich, Academic Press, Inc., $260 \mathrm{pp}$.

Nakanishi, K.K., S.R. Taylor, N.C. Burr, and P.W. Rodgers (1983) Broadband Seismograms from Merged Midand Short-Period RSTN Data, Lawrence Livermore National Laboratory, Livermore, CA, UCID19884.

Owens, T. J., S. R. Taylor, and G. Zandt (1987), "Crustal Structure at Regional Seismic Test Network Stations Determined from Inversion of Broadband Teleseismic $P$ Waveforms," Bull. Seismol. Soc. Am. 77, 631-662.

Prodehl, C.(1970), "Seismic Refraction Study of Crustal Structure in the Western United States," Bull. Geol. Soc. Am. 81, 2629-2646.

Taylor, S. R., and B. J. Qualheim (1983), RSTN Site Descriptions, Lawrence Livermore National Laboratory, Livermore, CA, UCID-19769.att, S. R., and T. C. Bache (1988), "Locating Events with a Sparse Network of Regional Arrays," Bull. Seismol. Soc. Am. 78, 780-798.

\section{Acknowledgments}

I would like to thank Steve Jarpe for significant help in data processing, Steve Jarpe and Keith Nakanishi for many helpful discussions and rewiew, Joe Tull for developing SAC, and Elaine Price for editoral revisions. 
Table 1

Station
rssd
rsny
rson
rsnt
rscp

yrdy hrmn

822672219:16.9

822682317:05.4

822760431:05.0

822830435:42.1

822871253:46.3

822960549:07.1

822961228:43.5

822991531:32.6

823190258:22.9

823251635:28.3

823261009:01.4

823302002:04.3

823381608:32.0

823510547:41.0

823511019:14.0

823570706:39.3

823600811:51.1

823600846:22.2

830060235:04.0

830102131:27.0

830170819:42.0

830191735:51.0

830190230:40.3

830200916:45.0

830201417:21.0

830242115:01.1

830261407:44.8

830272209:35.0

830361308:19.5

830421546:56.0

830431425:19.0

830441344:44.0*

830470622:09.3

830470714:07.4

830472056:33.0

830540851:27.0

830612323:19.4

830630632:18.6

830730911:20.0*

830760725:56.6*

830790300:02.0

830810455:04.0
Latitude

44.1204

44.5483

50.8589

62.4797

35.6000
Longitude 104.0362

074.5300

093.7022

114.5917

085.5686

Table 2

Events for Study

Latitude

35.689

35.210

51.250

49.950

36.102

65.710

47.149

47.000

43.007

35.190

39.739

46.269

47.500

43.700

49.310

42.720

64.470

64.990

52.120

46.820

48.960

49.110

35.178

48.720

47.460

43.966

32.728

36.054

34.700

48.990

55.830

42.232

48.539

45.927

51.320

36.192

34.302

44.214

50.820

47.526

51.600

51.640

Longitude Depth

$084.247 \quad 10.0$

$\underline{\mathrm{Mag}}$

master

$\begin{array}{lll}092.230 & 04.0 & 3.5\end{array}$

$\begin{array}{lll}062.810 & 18.0 & 3.3\end{array}$

$\begin{array}{lll}063.530 & 18.0 & 2.6\end{array}$

$\begin{array}{lll}102.571 & 05.0 & 3.9\end{array}$

$\begin{array}{lll}090.370 & 18.0 & 2.9\end{array}$

$\begin{array}{lll}112.712 & 05.0 & 3.6\end{array}$

$\begin{array}{lll}066.600 & 05.0 & 3.6\end{array}$

$\begin{array}{lll}097.850 & 05.0 & 4.3\end{array}$

$\begin{array}{lll}092.230 & 03.0 & 3.4\end{array}$

$\begin{array}{lll}107.581 & 05.0 & 2.9\end{array}$

$\begin{array}{lll}111.988 & 05.0 & 3.4\end{array}$

$\begin{array}{lll}070.200 & 18.0 & 4.0\end{array}$

$\begin{array}{lll}074.210 & 06.0 & 2.0\end{array}$

$\begin{array}{lll}085.710 & 01.0 & 2.7\end{array}$

$\begin{array}{lll}081.480 & 09.0 & 2.9\end{array}$

$\begin{array}{lll}090.720 & 18.0 & 2.8\end{array}$

$\begin{array}{lll}086.610 & 18.0 & 3.0\end{array}$

$\begin{array}{lll}106.930 & 01.0 & 2.7\end{array}$

$\begin{array}{lll}078.840 & 18.0 & 3.3\end{array}$

$\begin{array}{lll}067.250 & 15.0 & 2.5\end{array}$

$\begin{array}{lll}066.980 & 18.0 & 4.1\end{array}$

$\begin{array}{lll}092.220 & 11.0 & 3.5\end{array}$

$\begin{array}{lll}083.450 & 18.0 & 3.0\end{array}$

$\begin{array}{lll}067.860 & 17.0 & 3.1\end{array}$

$\begin{array}{lll}073.358 & 11.0 & 2.4\end{array}$

$\begin{array}{lll}083.375 & 05.0 & 3.5\end{array}$

$\begin{array}{lll}083.619 & 15.0 & 2.6\end{array}$

$\begin{array}{lll}088.370 & 03.0 & 2.9\end{array}$

$\begin{array}{lll}068.300 & 18.0 & 3.6\end{array}$

$\begin{array}{lll}082.740 & 18.0 & 2.6\end{array}$

$\begin{array}{lll}105.729 & 05.0 & 4.0\end{array}$

$\begin{array}{lll}112.373 & 14.0 & 3.5\end{array}$

$\begin{array}{lll}111.497 & 05.0 & 3.7\end{array}$

$\begin{array}{lll}067.930 & 18.0 & 2.0\end{array}$

$\begin{array}{lll}089.604 & 09.0 & 3.6\end{array}$

$\begin{array}{lll}106.892 & 08.0 & 4.1\end{array}$

$\begin{array}{lll}099.409 & 05.0 & \mathbf{4 . 4}\end{array}$

$\begin{array}{lll}074.900 & 18.0 & 3.7\end{array}$

$\begin{array}{lll}112.702 & 05.0 & 4.2\end{array}$

$\begin{array}{lll}115.070 & 18.0 & 2.2\end{array}$

$\begin{array}{lll}115.030 & 05.0 & 3.6\end{array}$

Churchill III

$$
\begin{aligned}
& \text { Superior } \\
& \text { Churchill II }
\end{aligned}
$$




\begin{tabular}{|c|c|c|c|c|c|}
\hline $830811154: 45.0$ & 48.180 & 114.080 & 18.0 & 2.8 & \\
\hline $830820141: 29.2$ & 47.930 & 114.000 & 18.0 & 3.2 & \\
\hline $830821147: 23.0$ & 65.690 & 111.560 & 18.0 & 3.3 & \\
\hline $830840247: 11.1$ & 35.345 & 082.462 & 09.0 & 3.2 & \\
\hline $830930455: 21.2$ & 35.448 & 102.321 & 05.0 & 3.4 & \\
\hline $831111702: 29.0$ & 53.520 & 075.580 & 02.0 & 2.5 & \\
\hline $831142159: 30.0$ & 53.570 & 075.700 & 02.0 & 3.6 & \\
\hline $831181035: 03.2$ & 54.000 & 081.000 & 18.0 & 2.9 & \\
\hline $831200734: 20.1$ & 33.316 & 106.438 & 07.0 & 3.5 & \\
\hline $831260614: 46.9$ & 42.955 & 102.198 & 05.0 & 3.3 & \\
\hline $831331726: 02.0$ & 47.000 & 066.600 & 05.0 & 3.6 & \\
\hline $831332340: 57.0$ & 47.000 & 066.600 & 05.0 & 4.1 & \\
\hline $831350516: 21.6^{*}$ & 38.770 & 089.570 & 09.0 & 4.3 & Central \\
\hline $831360201: 57.0$ & 47.700 & 069.930 & 10.0 & 4.0 & \\
\hline 831430149 & 46.550 & 080.830 & 01.0 & 2.6 & \\
\hline $831490545: 49.8$ & 44.502 & 070.415 & 02.0 & 3.9 & \\
\hline $831530630: 23.0$ & 47.450 & 070.220 & 10.0 & 3.4 & \\
\hline $831542239: 00.6$ & 38.140 & 088.410 & 22.0 & 2.7 & \\
\hline $831621347: 58.0$ & 47.000 & 066.600 & 05.0 & 3.4 & \\
\hline $831671636: 36.0$ & 44.740 & 072.550 & 01.0 & 2.7 & \\
\hline $831721358: 28.5$ & 35.190 & 092.180 & 04.0 & 2.5 & \\
\hline $831730404: 14.4$ & 35.230 & 092.220 & 04.0 & 2.1 & \\
\hline $831790805: 49.0$ & 47.050 & 066.690 & 05.0 & 3.3 & \\
\hline $832241408: 47.6$ & 44.970 & 067.680 & 12.0 & 3.6 & \\
\hline $832261908: 30.7$ & 38.359 & 107.402 & 05.0 & 3.4 & \\
\hline $832291403: 15.0^{*}$ & 38.472 & 082.772 & 12.0 & 3.5 & Green2 \\
\hline $832671657: 45.7$ & 40.789 & 108.837 & 05.0 & 4.1 & \\
\hline $832801018: 46.1^{*}$ & 43.938 & 074.258 & 13.0 & 5.1 & Green 1 \\
\hline $832801039: 38.5$ & 43.952 & 074.258 & 08.0 & 3.5 & \\
\hline $832840410: 55.0$ & 45.210 & 075.770 & 15.0 & 4.2 & \\
\hline 832891940:50.8 & 30.243 & 093.393 & 05.0 & 3.8 & \\
\hline $833051016: 52.0$ & 45.680 & 073.900 & 18.0 & 3.5 & \\
\hline 833062003:58.8 & 43.418 & 110.921 & 05.0 & 3.5 & \\
\hline $833100902: 19.8$ & 32.937 & 080.159 & 10.0 & 3.1 & \\
\hline $833131353: 12.9$ & 43.716 & 110.200 & 05.0 & 3.6 & \\
\hline 833191233:12.1 & 43.016 & 105.955 & 05.0 & 3.0 & \\
\hline $833211532: 18.0$ & 47.000 & 066.600 & 05.0 & 3.7 & \\
\hline $833231622: 20.1$ & 41.830 & 081.090 & 18.0 & 2.5 & \\
\hline 833351654 & 38.710 & 090.840 & 10.0 & 3.2 & \\
\hline 833381048:33.7 & 45.190 & 069.140 & 01.0 & 3.4 & \\
\hline $833432052: 10.5$ & 33.183 & 092.704 & 05.0 & 3.0 & \\
\hline $833542252: 23.7$ & 43.294 & 110.767 & 05.0 & 4.5 & \\
\hline 833542321:52.5 & 43.268 & 110.826 & 05.0 & 3.5 & \\
\hline $833551504: 44.0$ & 45.220 & 073.980 & 11.0 & 3.1 & \\
\hline $833552025: 22.2$ & 46.939 & 113.542 & 05.0 & 3.0 & \\
\hline $833561856: 03.9$ & 43.224 & 110.802 & 05.0 & 3.4 & \\
\hline $833621224: 21.0$ & 47.010 & 076.330 & 18.0 & 3.4 & \\
\hline $840840521: 51.8$ & 43.329 & 110.782 & 05.0 & 2.8 & \\
\hline $841080444: 43.8$ & 38.381 & 088.435 & 20.0 & 3.4 & \\
\hline $841241825: 35.4$ & 39.291 & 107.229 & 05.0 & 2.5 & \\
\hline $841270200: 56.6$ & 39.335 & 107.249 & 05.0 & 2.1 & \\
\hline$\underline{841380911: 20.2}$ & 39.337 & 107.245 & 05.0 & 2.4 & \\
\hline
\end{tabular}


Table 3

Weights based upon signal to noise ratio signal to noise ratio $10: 1<S N R$

$5: 1<S N R \leq 10: 1$

$2: 1<S N R \leq 5: 1$

$1: 1<S N R \leq 2: 1$

$S N R \leq 1: 1$

weight

0

1

2

3

4

Table 4

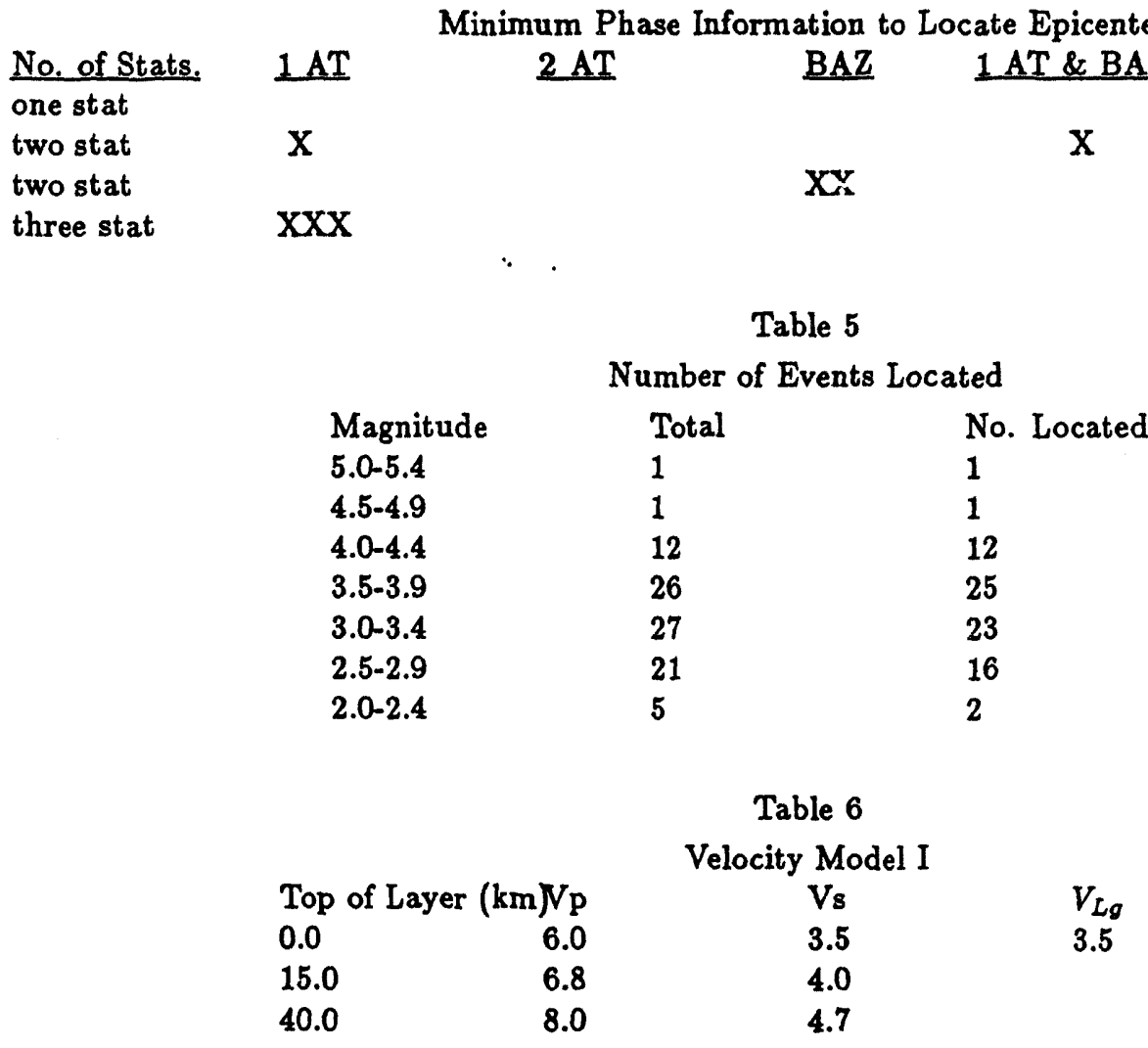

Table 7

Travel Time Residuals Squared

\begin{tabular}{|c|c|c|c|c|c|c|c|c|c|}
\hline Station & $\mathrm{Pg}$ & $\underline{N}$ & $\underline{P n}$ & $\underline{N}$ & $\underline{\mathrm{Sn}}$ & $\underline{N}$ & $\underline{L g}$ & $\underline{N}$ & BAZ \\
\hline RSON & 532.0 & 35 & 132.7 & 22 & 432.8 & 27 & 276.2 & 34 & 4707.0 \\
\hline RSNT & 532.0 & $35^{*}$ & 120.0 & 09 & 060.0 & 08 & 009.3 & 05 & 11255.0 \\
\hline RSSD & 201.8 & 13 & 130.9 & 19 & 095.1 & 14 & 338.0 & 31 & 5143.0 \\
\hline RSCP & 175.1 & 12 & 089.8 & 15 & 204.2 & 16 & 257.6 & 29 & $11255.0^{* *}$ \\
\hline RSNY & 156.5 & 10 & 227.5 & 17 & 160.1 & 21 & 546.2 & 51 & 1405.0 \\
\hline
\end{tabular}


Table 8

Location Summary - Average Velocity Structure

\begin{tabular}{|c|c|c|c|c|c|c|c|}
\hline yrdayhrmn sec & Latitude & Longitude. & $\underline{\text { Mag }}$ & $\underline{\mathrm{St}}$ & Variance & Loc error & $95 \%$ error \\
\hline $822672219: 18.4$ & 35.985 & 84.411 & 3.4 & 3 & 4.29 & 36.1 & 111.6 \\
\hline $822682317: 07.0$ & 35.386 & 92.055 & 3.5 & 4 & 5.49 & 25.1 & 77.9 \\
\hline 822760431:11.6 & 49.883 & 59.619 & 3.3 & 2 & 5.10 & 272.5 & 1557.1 \\
\hline 822871253:53.2 & $36.2 ؟ 4$ & 101.926 & 3.9 & 4 & 5.33 & 61.7 & 73.4 \\
\hline $822960549: 15.6$ & 73.076 & 117.123 & 2.9 & 1 & 10.59 & 1316.6 & 2545.2 \\
\hline 822961228:51.9 & 47.135 & 112.067 & 3.6 & 2 & 6.70 & 49.0 & 106.7 \\
\hline $822991531: 35.8$ & 46.345 & 66.563 & 3.6 & 2 & 4.98 & 72.9 & 1528.5 \\
\hline 823190258:18.3 & 43.043 & 97.585 & 4.3 & 4 & 5.76 & 22.0 & 29.5 \\
\hline $823251635: 28.8$ & 35.420 & 92.083 & 3.4 & 3 & 6.57 & 28.7 & 115.3 \\
\hline 823261008:52.3 & 41.951 & 111.403 & 2.9 & 1 & 10.83 & 405.0 & 1038.7 \\
\hline $823302002: 03.9$ & 46.692 & 111.726 & 3.4 & 3 & 4.40 & 51.1 & 97.6 \\
\hline 823381608:33.2 & 47.869 & 71.201 & 4.0 & 5 & 6.17 & 85.6 & 105.6 \\
\hline $823510547: 10.5$ & 51.343 & 67.170 & 2.0 & 1 & 11.54 & 1000.6 & 2415.2 \\
\hline $823511019: 39.5$ & 52.994 & 87.633 & 2.7 & 1 & 10.38 & 431.4 & 979.2 \\
\hline $823570706: 14.2$ & 44.066 & 83.282 & 2.9 & 1 & 8.20 & 208.9 & 658.0 \\
\hline $823600811: 45.2$ & 66.945 & 93.038 & 2.8 & 1 & 10.59 & 296.0 & 2303.5 \\
\hline $823600846: 51.9$ & 52.864 & 103.690 & 2.8 & 1 & 8.45 & 1490.4 & 1979.8 \\
\hline 830060234:52.9 & 51.741 & 107.245 & 2.7 & 3 & 8.65 & 47.4 & 106.0 \\
\hline $830102131: 24.0$ & 47.408 & 78.819 & 3.3 & 2 & 7.37 & 65.4 & 598.2 \\
\hline $830170820: 57.2$ & 42.263 & 69.077 & 2.5 & 1 & 2.93 & 757.7 & 455.9 \\
\hline $830191735: 52.7$ & 49.348 & 68.231 & 4.1 & 4 & 4.62 & 94.9 & 88.8 \\
\hline $830190230: 45.5$ & 35.681 & 91.862 & 3.5 & 4 & 5.36 & 64.6 & 88.3 \\
\hline $830200916: 47.9$ & 49.012 & 82.898 & 3.1 & 2 & 6.36 & 51.9 & 569.7 \\
\hline $830201417: 24.5$ & 47.451 & 68.307 & 3.1 & 1 & 4.99 & 33.8 & 802.6 \\
\hline $830261407: 46.4$ & 33.082 & 83.506 & 3.5 & 3 & 2.54 & 41.1 & 43.1 \\
\hline $830272209: 34.3$ & 37.172 & 85.042 & 2.6 & 1 & 4.98 & 177.6 & 262.3 \\
\hline $830361308: 16.5$ & 34.707 & 88.345 & 2.9 & 2 & 3.18 & 3.1 & 78.5 \\
\hline $830421546: 57.0$ & 49.227 & 69.078 & 3.6 & 3 & 4.10 & 62.6 & 95.0 \\
\hline 830431425:53.6 & 53.852 & 84.890 & 2.6 & 1 & 7.65 & 260.0 & 1218.6 \\
\hline 830441344:35.8* & 42.361 & 106.355 & 4.0 & 5 & 5.90 & 53.6 & 75.9 \\
\hline 830470622:03.1 & 48.622 & 112.426 & 3.5 & 3 & 7.10 & 9.8 & 109.9 \\
\hline $830470714: 09.2$ & 46.302 & 111.201 & 3.7 & 3 & 4.91 & 47.6 & 90.4 \\
\hline $830540851: 34.0$ & 37.091 & 88.895 & 3.6 & 4 & 5.48 & 118.1 & 93.0 \\
\hline 830612323:33.9 & 34.679 & 106.159 & 4.1 & 3 & 4.56 & 79.2 & 70.0 \\
\hline $830630632: 12.5$ & 44.132 & 99.096 & 4.4 & 4 & 5.93 & 26.6 & 26.0 \\
\hline 830730911:23.4* & 50.460 & 75.901 & 3.6 & 5 & 5.39 & 81.4 & 100.1 \\
\hline 830760726:04.6* & 47.430 & 111.759 & 4.2 & 3 & 6.31 & 71.7 & 105.3 \\
\hline $830810454: 55.8$ & 51.567 & 115.424 & 3.6 & 2 & 5.41 & 28.5 & 101.1 \\
\hline $830820141: 55.7$ & 47.381 & 112.325 & 3.2 & 1 & 5.29 & 139.8 & 1054.2 \\
\hline $830840247: 04.7$ & 35.656 & 82.281 & 3.2 & 2 & 2.73 & 38.2 & 81.6 \\
\hline $830930455: 20.7$ & 35.455 & 102.258 & 3.4 & 3 & 5.66 & 6.2 & 94.2 \\
\hline $831142159: 37.5$ & 53.155 & 75.910 & 3.6 & 2 & 6.16 & 48.3 & 163.4 \\
\hline $831181035: 17.0$ & 52.802 & 81.891 & 2.9 & 2 & 4.83 & 146.0 & 111.9 \\
\hline 831200734:22.9 & 33.469 & 106.600 & 3.5 & 3 & 2.78 & 22.6 & 46.9 \\
\hline 831260614:54.2 & 43.447 & 102.489 & 3.3 & 2 & 6.58 & 59.5 & 98.3 \\
\hline 831331726:16.0 & 47.307 & 67.759 & 3.6 & 2 & 6.39 & 94.3 & 98.4 \\
\hline $831332340: 46.7$ & 46.669 & 65.958 & 4.1 & 5 & 7.41 & 61.3 & 133.6 \\
\hline 831350516:19.0* & 38.989 & 89.440 & 4.3 & 4 & 6.38 & 26.7 & 36.5 \\
\hline 831360202:01.0 & 47.832 & 70.757 & 4.0 & 5 & 6.13 & 63.7 & 100.5 \\
\hline 831490545:58.4 & 44.792 & 71.448 & 3.9 & 5 & 5.57 & 88.0 & 89.0 \\
\hline $831530630: 44.5$ & 47.240 & 72.240 & 3.4 & 3 & 5.86 & 154.4 & 102.5 \\
\hline
\end{tabular}




\begin{tabular}{|c|c|c|c|c|c|c|c|}
\hline $831621348: 04.1$ & 47.312 & 67.296 & 3.4 & 2 & 3.91 & 63.2 & 78.0 \\
\hline $831671636: 36.3$ & 44.601 & 72.582 & 2.6 & 1 & 4.10 & 15.9 & 971.8 \\
\hline $831721358: 18.3$ & 36.209 & 91.378 & 2.5 & 2 & 5.21 & 134.3 & 185.7 \\
\hline $831730404: 37.1$ & 34.145 & 88.115 & 2.1 & 1 & 5.18 & 394.6 & 265.4 \\
\hline $831790805: 52.4$ & 47.469 & 67.266 & 3.3 & 2 & 3.79 & 63.8 & 76.7 \\
\hline $832241408: 49.6$ & 45.741 & 68.153 & 3.6 & 2 & 4.66 & 93.3 & 82.0 \\
\hline $832261908: 30.5$ & 40.514 & 111.636 & 3.4 & 1 & 10.89 & 435.7 & 1290.4 \\
\hline $832291403: 17.6^{*}$ & 38.988 & 82.920 & 3.5 & 3 & 5.48 & 58.7 & 74.8 \\
\hline $832671657: 44.3$ & 39.477 & 106.540 & 4.1 & 2 & 4.80 & 243.9 & 694.7 \\
\hline $832801018: 45.3^{*}$ & 44.112 & 75.040 & 5.1 & 5 & 6.17 & 65.6 & 65.4 \\
\hline $832801039: 44.4$ & 44.174 & 74.908 & 5.1 & 3 & 7.84 & 58.3 & 77.7 \\
\hline $8328\{0410: 49.5$ & 45.132 & 76.168 & 4.2 & 4 & 4.86 & 32.5 & 47.0 \\
\hline $832891940: 46.5$ & 29.861 & 93.461 & 3.8 & 3 & 5.19 & 42.8 & 78.0 \\
\hline $833051016: 46.7$ & 45.939 & 74.246 & 3.5 & 2 & 3.42 & 39.4 & 136.1 \\
\hline $833100902: 26.0$ & 33.532 & 80.278 & 3.1 & 2 & 3.61 & 66.9 & 64.8 \\
\hline $833131353: 13.6$ & 44.244 & 109.584 & 3.6 & 2 & 4.49 & 76.7 & 110.8 \\
\hline $833191233: 13.6$ & 43.811 & 106.422 & 3.0 & 2 & 2.30 & 96.0 & 75.8 \\
\hline $833211532: 24.2$ & 47.398 & 67.343 & 3.7 & 2 & 3.86 & 71.6 & 75.2 \\
\hline $833381048: 34.7$ & 45.929 & 69.622 & 3.4 & 2 & 4.62 & 90.3 & 112.1 \\
\hline $833542252: 26.6$ & 43.906 & 110.507 & 4.5 & 3 & 6.14 & 71.2 & 118.1 \\
\hline $833542321: 58.9$ & 43.822 & 110.302 & 3.5 & 2 & 5.73 & 74.7 & 371.2 \\
\hline $833551504: 42.7$ & 44.655 & 73.412 & 3.1 & 1 & 3.40 & 77.2 & 237.3 \\
\hline $833552025: 31.2$ & 46.590 & 113.025 & 3.0 & 2 & 8.07 & 55.3 & 230.9 \\
\hline $833561856: 11.3$ & 44.238 & 110.346 & 3.4 & 2 & 5.71 & 118.5 & 325.2 \\
\hline $833621224: 14.7$ & 47.028 & 76.551 & 3.4 & 3 & 3.55 & 17.0 & 60.9 \\
\hline $840840521: 54.4$ & 45.222 & 110.314 & 2.8 & 1 & 5.29 & 213.6 & 760.6 \\
\hline $841080444: 39.1$ & 38.548 & 88.195 & 3.4 & 4 & 5.53 & 28.0 & 46.5 \\
\hline $841241825: 30.0$ & 42.250 & 110.918 & 2.5 & 1 & 5.29 & 452.5 & 678.3 \\
\hline $841270200: 58.1$ & 42.056 & 110.590 & 2.1 & 1 & 5.29 & 413.3 & 681.6 \\
\hline $841380911: 22.5$ & 39.615 & 107.361 & 2.4 & 1 & 5.29 & 32.4 & 860.5 \\
\hline
\end{tabular}


Table 9

North American Crustal Models

$\begin{array}{lcllclll}\text { Province } & \text { upper crust } & V_{p} & V_{s} & \text { lower crust } & V_{p} & V_{s} & \text { mantle } \\ \text { Churchill } & 12 \mathrm{~km} & 5.9 & 3.6(\mathrm{~km} / \mathrm{sec}) & 25 \mathrm{~km} & 6.9 & 4.0(\mathrm{~km} / \mathrm{sec}) & 7.94 .6(\mathrm{~km} / \mathrm{sec}) \\ \text { Superior } & 14 & 6.3 & 3.7 & 20 & 6.9 & 4.0 & 8.14 .7 \\ \text { Central } & 20 & 6.1 & 3.6 & 19 & 6.9 & 4.0 & 8.14 .6 \\ \text { Grenville } & 21 & 6.3 & 3.6 & 18 & 6.9 & 3.9 & 8.24 .7\end{array}$

Table 10

RSON

Province

Churchill I

Churchill II

Churchill III

Superior

Central

Grenville I

Grenville II
$\mathrm{Pg}$ Pn Sn Lg Bz

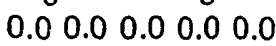
$-3.4-3.6 \quad 2.01 .90 .0$ 0.0-8.1-0.1 1.20 .0 $0.0-0.35 .13 .10 .0$ $\begin{array}{lllll}0.0-0.2-3.0 & 1.7 & 0.0\end{array}$ $0.0-1.6 \quad 3.2-3.10 .0$ $0.0-6.8-4.0-0.8 \quad 0.0$

Station Corrections

RSNT

Pg Pn Sn Lg Bz 0.00 .00 .00 .00 .0 0.00 .00 .00 .00 .0 0.00 .07 .91 .60 .0 $\begin{array}{llllllllll}0.0 & 0.0 & 0.0 & 0.0 & 0.0\end{array}$ $\begin{array}{llllllllllllll}0.0 & 0.0 & 0.0 & 0.0 & 0.0\end{array}$ $\begin{array}{lllllllllllll}0.0 & 1.2-3.6 & 0.0 & 0.0\end{array}$ $\begin{array}{lllllllllllll}0.0 & 0.0 & 0.0 & 0.0 & 0.0\end{array}$
RSSD

$\mathrm{Pg} \mathrm{Pn} \mathrm{Sn} \mathrm{Lg} \mathrm{Bz}$ $\begin{array}{llllllllllll}0.0 & 0.0 & 0.0 & 0.0 & 0.0\end{array}$ $\begin{array}{llllllllll}4.3 & 3.1 & 0.0-3.6 & 0.0\end{array}$ $\begin{array}{lllll}-2.9 & 0.0 & 0.0 & 5.3 & 0.0\end{array}$ $0.0 \quad 0.0-8.1 \quad 19.8 \quad 0.0$ $0.0-0.2-1.8 \quad 0.6 \quad 0.0$ $0.0-4.6 \quad 17.5 \quad 5.40 .0$ 0.00 .04 .54 .10 .0
RSCP

$\mathrm{Pg} \mathrm{Pn} \mathrm{Sn} \mathrm{Lg} \mathrm{Bz}$ $\begin{array}{llllllllllll}0.0 & 0.0 & 0.0 & 0.0 & 0.0\end{array}$ $0.0-0.10 .0-0.10 .0$ $0.0-1.81 .99 .30 .0$ $0.0 \quad 0.00 .06 .90 .0$ -3.5 2.4 3.7-2.7 0.0 $\begin{array}{llll}-2.5 & 1.7 & 5.1-1.6 & 0.0\end{array}$ $\begin{array}{llllllllllllll}0.0 & 0.0 & 0.0 & 0.0 & 0.0\end{array}$
RSNY

$\mathrm{Pg}$ Pn Sn Lg Bz

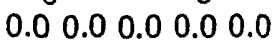
$0.0 \quad 0.00 .0 \quad 0.00 .0$ $\begin{array}{llllllllllll}0.0 & 0.0 & 0.0 & 5.3 & 0.0\end{array}$ $\begin{array}{lllll}0.0 & 3.6 & 0.8-3.2 & 0.0\end{array}$ $0.02 .01 .1-2.40 .0$ $2.7 \quad 1.0-0.53 .70 .0$ $0.0-2.32 .41 .70 .0$ 
Table 11

\begin{tabular}{|c|c|c|c|c|c|c|c|c|c|c|}
\hline \multicolumn{9}{|c|}{ Churchill I } & $B A Z^{*}$ & $\underline{N}$ \\
\hline RSON & 000.0 & 00 & 027.0 & 10 & 090.0 & 00 & 027.9 & 08 & 0661.0 & 04 \\
\hline RSNT & 000.0 & 00 & 109.8 & 04 & 000.0 & 00 & 106.4 & 05 & 0000.0 & 00 \\
\hline RSSD & 268.2 & 09 & 023.9 & 08 & 005.2 & 03 & 098.5 & 11 & 0321.0 & 11 \\
\hline RSCP & 000.0 & 00 & 000.0 & 00 & 000.0 & Do & 000.0 & 00 & 0000.0 & 00 \\
\hline RSNY & 000.0 & 00 & 000.0 & 00 & 000.0 & 00 & 000.0 & 00 & 0000.0 & 00 \\
\hline \multicolumn{11}{|c|}{ Churchill II } \\
\hline $\mathrm{RSON}$ & 000.0 & 00 & 027.0 & 10 & 000.0 & 00 & 027.9 & 08 & 0661.0 & 04 \\
\hline RSNT & 000.0 & 00 & 109.8 & 04 & 000.0 & 00 & 106.4 & 05 & 0000.0 & 00 \\
\hline RSSD & 268.2 & 09 & 023.9 & 08 & 005.2 & 03 & 098.5 & 11 & 0321.0 & 11 \\
\hline RSCP & 000.0 & 00 & 000.0 & 00 & 000.0 & 00 & 000.0 & 00 & 0000.0 & 00 \\
\hline RSNY & 000.0 & 00 & 000.0 & 00 & 000.0 & 00 & 000.0 & 00 & 0000.0 & 00 \\
\hline \multicolumn{10}{|c|}{ Churchill III } & \\
\hline $\mathrm{RSON}$ & 000.0 & 00 & 063.2 & 05 & 056.2 & 04 & 037.7 & 04 & 0000.0 & 00 \\
\hline RSNT & 000.0 & 00 & 000.0 & 00 & 000.0 & 00 & 000.0 & 00 & 0000.0 & 00 \\
\hline RSSD & 052.5 & 08 & 013.1 & 04 & 054.0 & 04 & 168.2 & 08 & 0000.0 & 00 \\
\hline RSCP & 000.0 & 00 & 005.5 & 03 & 000.0 & 00 & 148.4 & 04 & 0000.0 & 00 \\
\hline \multicolumn{11}{|l|}{ Superior } \\
\hline RSON & 000.0 & 00 & 000.0 & 00 & 000.0 & 00 & 000.0 & 00 & 0058.0 & 02 \\
\hline RSNT & 000.0 & 00 & 000.0 & 00 & 000.0 & 00 & 000.0 & 00 & 0000.0 & 00 \\
\hline RSSD & 000.0 & 00 & 000.0 & 00 & 000.0 & 00 & 000.0 & 00 & 0032.4 & 01 \\
\hline RSCP & 000.0 & 00 & 000.0 & 00 & 000.0 & 00 & 000.0 & 00 & 0000.0 & 00 \\
\hline $\begin{array}{l}\text { RSNY } \\
\text { Central }\end{array}$ & 000.0 & 00 & 000.0 & 00 & 000.0 & 00 & $0 \mathrm{C5} .0$ & 03 & 0019.0 & 02 \\
\hline RSON & 000.0 & 00 & 067.9 & 04 & 155.8 & 05 & 043.7 & 05 & 0000.0 & 00 \\
\hline RSNT & 000.0 & 00 & 000.0 & 00 & 000.0 & 00 & 000.0 & 00 & 0000.0 & 00 \\
\hline RSSD & 000.0 & 00 & 016.4 & 04 & 014.8 & 05 & 080.5 & 07 & 0000.0 & 00 \\
\hline RSCP & 048.8 & 07 & 038.5 & 04 & 016.7 & 06 & 012.5 & 04 & 0000.0 & 00 \\
\hline RSNY & 000.0 & 00 & 000.0 & 00 & 000.0 & 00 & 062.5 & 04 & 0000.0 & 00 \\
\hline \multicolumn{11}{|c|}{ Grenville I } \\
\hline RSON & 000.0 & 00 & 051.2 & 05 & 035.6 & 04 & 063.5 & 13 & 0162.8 & 06 \\
\hline RSNT & 000.0 & 00 & 000.0 & 00 & 128.0 & 04 & 000.0 & 00 & 0000.0 & 00 \\
\hline RSSD & 000.0 & 00 & 000.0 & 00 & 103.2 & 03 & 108.5 & 04 & 0000.0 & 00 \\
\hline RSCP & 000.0 & 00 & 022.0 & 04 & 000.0 & 00 & 197.2 & 06 & 0000.0 & 00 \\
\hline RSNY & 386.8 & 09 & 110.3 & 12 & 098.5 & 11 & 074.5 & 11 & 0016.3 & 04 \\
\hline \multicolumn{11}{|c|}{ Grenville II } \\
\hline RSON & 000.0 & 00 & 000.0 & 00 & 000.0 & 00 & 000.0 & 00 & 0000.0 & $\infty$ \\
\hline RSNT & 000.0 & 00 & 000.0 & 00 & 000.0 & 00 & 000.0 & 00 & 0000.0 & 00 \\
\hline RSSD & 000.0 & 00 & 000.0 & 00 & 000.0 & 00 & 009.2 & 03 & 0000.0 & 00 \\
\hline RSCP & 010.2 & 03 & 000.0 & 00 & 000.0 & 00 & 007.2 & 05 & 0000.0 & 00 \\
\hline$\underline{\text { RSNY }}$ & 000.0 & 00 & 000.0 & 00 & 000.0 & 00 & 004.4 & 02 & 0000.0 & 00 \\
\hline
\end{tabular}


Table 12

Location Summary - Regional Velocity Structure

\begin{tabular}{|c|c|c|c|c|c|c|c|}
\hline yrdayhrmn sec & Latitude & Longitude & $\underline{\mathrm{Mag}}$ & $\underline{\text { St }}$ & Variance & Loc error & $95 \%$ error \\
\hline 822672219:16.5 & 35.558 & 84.192 & 3.4 & 3 & 4.46 & 15.4 & 88.2 \\
\hline 822682317:07.1 & 34.981 & 92.239 & 3.5 & 4 & 7.61 & 25.4 & 95.1 \\
\hline 822760431:07.5 & 49.962 & 59.214 & 3.3 & 2 & 6.11 & 292.1 & 1800.3 \\
\hline $822871253: 47.8$ & 35.977 & 102.290 & 3.9 & 4 & 5.68 & 28.9 & 74.0 \\
\hline $822960549: 18.9$ & 72.781 & 117.012 & 2.9 & 1 & 14.15 & 1299.7 & 3211.6 \\
\hline 822961228:36.6 & 47.059 & 113.211 & 3.6 & 2 & 9.73 & 39.2 & 138.7 \\
\hline 822991531:34.7 & 46.350 & 66.486 & 3.6 & 2 & 6.22 & 72.8 & 1804.4 \\
\hline $823190258: 19.4$ & 42.924 & 97.790 & 4.3 & 4 & 6.69 & 10.5 & 25.2 \\
\hline $823251635: 29.2$ & 35.037 & 92.199 & 3.4 & 3 & 6.97 & 17.2 & 120.5 \\
\hline $823261008: 53.8$ & 41.970 & 111.349 & 2.9 & 1 & 19.34 & 402.7 & 1539.9 \\
\hline 823302001:58.9 & 46.135 & 112.312 & 3.4 & 3 & 7.50 & 29.0 & 127.1 \\
\hline 823381608:38.9 & 47.309 & 70.907 & 4.0 & 5 & 16.15 & 57.4 & 178.3 \\
\hline $823510547: 32.1$ & 51.492 & 68.845 & 2.0 & 1 & 9.64 & 955.1 & 2918.9 \\
\hline $823511019: 39.5$ & 52.994 & 87.633 & 2.7 & 1 & 10.38 & 431.4 & 979.1 \\
\hline $823570706: 22.5$ & 44.109 & 82.811 & 2.9 & 1 & 21.52 & 188.2 & 1172.6 \\
\hline $823600811: 48.4$ & 66.867 & 93.726 & 2.8 & 1 & 14.15 & 301.1 & 2899.4 \\
\hline $823600846: 32.0$ & 52.162 & 103.116 & 2.8 & 1 & 12.55 & 1546.2 & 2516.2 \\
\hline $830060234: 51.5$ & 52.001 & 107.476 & 2.7 & 3 & 12.76 & 39.8 & 142.7 \\
\hline $830102131: 22.6$ & 46.563 & 79.207 & 3.3 & 2 & 5.34 & 40.1 & 1569.3 \\
\hline $830170820: 52.9$ & 42.184 & 68.905 & 2.5 & 1 & 4.98 & 764.0 & 689.6 \\
\hline $830191735: 58.4$ & 48.820 & 67.718 & 4.1 & 4 & 11.30 & 62.9 & 140.5 \\
\hline $830190230: 46.9$ & 35.367 & 92.001 & 3.5 & 4 & 7.26 & 28.8 & 103.0 \\
\hline $830200916: 51.5$ & 48.629 & 83.378 & 3.1 & 2 & 4.78 & 11.6 & 926.6 \\
\hline $830201417: 24.1$ & 47.456 & 68.295 & 3.1 & 1 & 6.36 & 32.8 & 960.3 \\
\hline $830261407: 49.3$ & 32.987 & 83.377 & 3.5 & 3 & 1.62 & 28.7 & 33.8 \\
\hline $830272209: 35.7$ & 37.172 & 85.042 & 2.6 & 1 & 1.90 & 177.6 & 186.4 \\
\hline $830361308: 20.0$ & 34.385 & 88.216 & 2.9 & 2 & 3.21 & 37.7 & 82.1 \\
\hline $830421546: 57.6$ & 48.754 & 68.403 & 3.6 & 3 & 5.12 & 27.3 & 104.6 \\
\hline $830431425: 11.4$ & 54.536 & 82.326 & 2.6 & 1 & 7.65 & 146.5 & 1434.5 \\
\hline $830441344: 46.6^{*}$ & 42.155 & 105.483 & 4.0 & 5 & 4.36 & 22.1 & 66.3 \\
\hline 830470622:01.2 & 48.468 & 112.836 & 3.5 & 3 & 11.01 & 35.1 & 152.2 \\
\hline $830470714: 04.7$ & 45.711 & 111.713 & 3.7 & 3 & 8.90 & 29.2 & 134.7 \\
\hline $830540851: 37.6$ & 36.915 & 88.945 & 3.6 & 4 & 7.96 & 99.5 & 107.0 \\
\hline $830612323: 17.8$ & 34.115 & 107.025 & 4.1 & 3 & 5.69 & 24.1 & 76.5 \\
\hline $830630632: 14.4$ & 44.055 & 99.315 & 4.4 & 4 & 6.84 & 19.2 & 18.8 \\
\hline 830730911:12.0* & 51.221 & 74.515 & 3.6 & 4 & 4.74 & 52.1 & 96.1 \\
\hline 830760725:53.1* & 47.367 & 112.879 & 4.2 & 3 & 9.00 & 22.3 & 133.4 \\
\hline 830810454:50.3 & 51.966 & 116.142 & 3.6 & 2 & 10.05 & 84.9 & 154.6 \\
\hline $830820141: 24.5$ & 47.952 & 114.082 & 3.2 & 1 & 13.96 & 6.2 & 2127.2 \\
\hline $83084.0247: 08.9$ & 35.559 & 82.356 & 3.2 & 2 & 1.09 & 25.5 & 20.7 \\
\hline $830930455: 15.9$ & 35.193 & 102.565 & 3.4 & 3 & 5.60 & 36.0 & 87.6 \\
\hline $831142159: 24.6$ & 54.003 & 74.516 & 3.6 & 2 & 5.55 & 91.7 & 161.4 \\
\hline $831181035: 11.2$ & 53.911 & 81.555 & 2.9 & 2 & 5. 7 & 37.8 & 105.5 \\
\hline $831200734: 02.5$ & 32.646 & 107.448 & 3.5 & 3 & 4.70 & 120.0 & 64.7 \\
\hline $831260615: 00.9$ & 44.989 & 104.698 & 3.3 & 2 & 5.32 & 302.1 & 145.7 \\
\hline $831331726: 19.2$ & 46.590 & 67.413 & 3.6 & 2 & 14.88 & $\mathbf{7 7 . 0}$ & 156.1 \\
\hline 831332340:58.4 & 46.940 & 66.763 & 4.1 & 5 & 6.96 & 14.2 & 107.0 \\
\hline $831350516: 22.8^{*}$ & 38.897 & 89.461 & 4.3 & 4 & 7.57 & 16.9 & 38.8 \\
\hline $831360201: 56.9$ & 47.561 & 69.992 & 4.0 & 5 & 15.57 & 16.2 & 161.9 \\
\hline $831490546: 00.0$ & 44.601 & 70.949 & 3.9 & 5 & 16.14 & 43.9 & 147.5 \\
\hline $831530630: 32.8$ & 47.166 & 70.959 & 3.4 & 3 & 5.26 & 64.2 & 97.6 \\
\hline
\end{tabular}




\begin{tabular}{|c|c|c|c|c|c|c|c|}
\hline $831621348: 02.9$ & 46.515 & 66.763 & 3.4 & 2 & 4.81 & 55.3 & 86.2 \\
\hline $831671636: 37.3$ & 44.598 & 72.739 & 2.6 & 1 & 5.27 & 21.8 & 1162.7 \\
\hline $831721358: 16.5$ & 35.405 & 91.674 & 2.5 & 2 & 3.11 & 51.8 & 106.4 \\
\hline $831730404: 18.9$ & 33.707 & 88.849 & 2.1 & 1 & 2.07 & 352.5 & 318.5 \\
\hline $831790805: 51.7$ & 46.705 & 66.740 & 3.3 & 2 & 4.67 & 38.6 & 79.0 \\
\hline $832241408: 51.4$ & 44.647 & 68.095 & 3.6 & 2 & 10.58 & 48.6 & 291.4 \\
\hline $832261908: 34.0$ & 40.613 & 111.451 & 3.4 & 1 & 16.42 & 428.6 & 1699.2 \\
\hline 832291403:21.3* & 38.812 & 82.610 & 3.5 & 3 & 5.86 & 40.3 & 80.8 \\
\hline $832671657: 46.5$ & 40.423 & 108.387 & 4.1 & 2 & 5.71 & 55.6 & 1713.1 \\
\hline $832801018: 44.8^{*}$ & 44.028 & 74.313 & 5.1 & 5 & 18.32 & 11.0 & 167.3 \\
\hline $837801039: 41.8$ & 44.028 & 74.160 & 5.1 & 3 & 9.54 & 12.8 & 137.5 \\
\hline $832840410: 51.2$ & 45.216 & 75.776 & 4.2 & 4 & 12.23 & 0.0 & 98.5 \\
\hline $832891940: 47.2$ & 29.734 & 93.591 & 3.8 & 3 & 6.99 & 59.5 & 90.0 \\
\hline $833051016: 45.8$ & 45.741 & 73.545 & 3.5 & 2 & 11.08 & 28.5 & 163.0 \\
\hline $833100902: 25.0$ & 33.204 & 80.309 & 3.1 & 2 & 4.04 & 32.6 & 63.5 \\
\hline $833131353: 01.5$ & 43.559 & 110.247 & 3.6 & 2 & 8.07 & 17.9 & 177.6 \\
\hline $833191233: 17.9$ & 43.278 & 105.917 & 3.0 & 2 & 2.44 & 29.3 & 107.2 \\
\hline $833211532: 22.3$ & 46.631 & 66.754 & 3.7 & 2 & 4.73 & 42.7 & 85.5 \\
\hline $833381048: 35.8$ & 45.020 & 69.423 & 3.4 & 2 & 10.34 & 29.2 & 200.4 \\
\hline $833542252: 13.4$ & 42.894 & 111.155 & 4.5 & 3 & 9.90 & 54.6 & 147.2 \\
\hline $833542321: 42.4$ & 42.151 & 110.700 & 3.5 & 2 & 8.97 & 124.5 & 742.0 \\
\hline $833551504: 42.1$ & 44.645 & 73.517 & 3.1 & 1 & 19.89 & 73.6 & 615.4 \\
\hline $833552025: 18.1$ & 46.653 & 114.104 & 3.0 & 2 & 10.32 & 53.4 & 277.2 \\
\hline $833561855: 55.3$ & 42.720 & 110.971 & 3.4 & 2 & 9.12 & 57.7 & 569.8 \\
\hline $833621224: 18.9$ & 47.053 & 75.850 & 3.4 & 3 & 11.71 & 36.7 & 140.7 \\
\hline $840840521: 27.7$ & 45.435 & 111.865 & 2.8 & 1 & 13.96 & 249.4 & 1709.6 \\
\hline $841080444: 44.1$ & 38.460 & 88.268 & 3.4 & 4 & 6.33 & 17.0 & 39.3 \\
\hline $841241825: 34.0$ & 42.307 & 110.734 & 2.5 & 1 & 2.53 & 446.6 & 506.9 \\
\hline $841270201: 02.4$ & 42.126 & 110.393 & 2.1 & 1 & 2.53 & 408.0 & 508.3 \\
\hline$\frac{841380911: 27.0}{* \text { master events }}$ & 39.766 & 107.258 & 2.4 & 1 & 2.53 & 47.6 & 644.1 \\
\hline
\end{tabular}




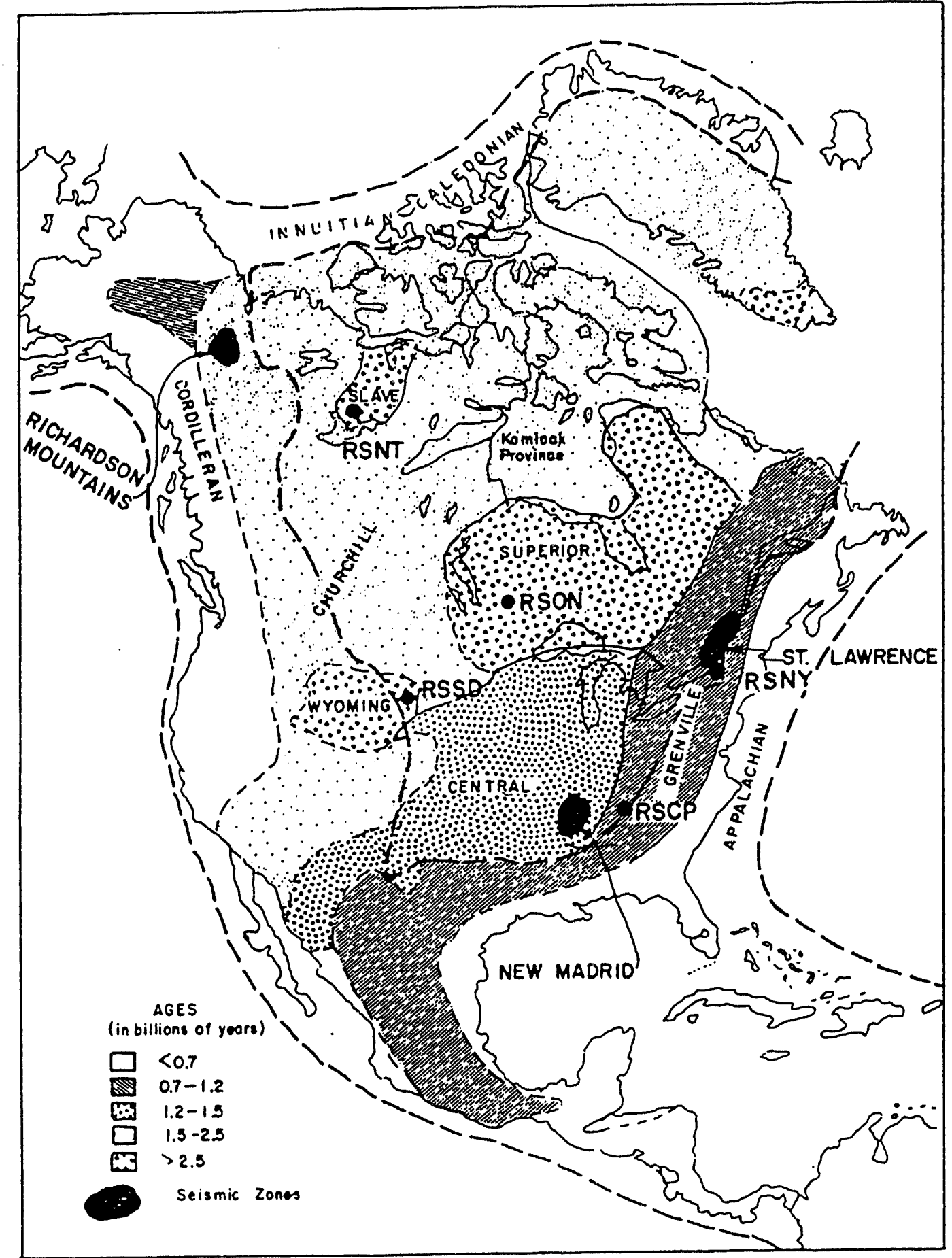

Figure 1. Locations of the RSTN stations, and major crustal provinces in North America (from Owens et. al., 1987). Major Precambrian regions are shown but excluding anorogenic provinces. Inner and outer boundaries of Phanerozoic belts are shown with the heavy dashed lines. 


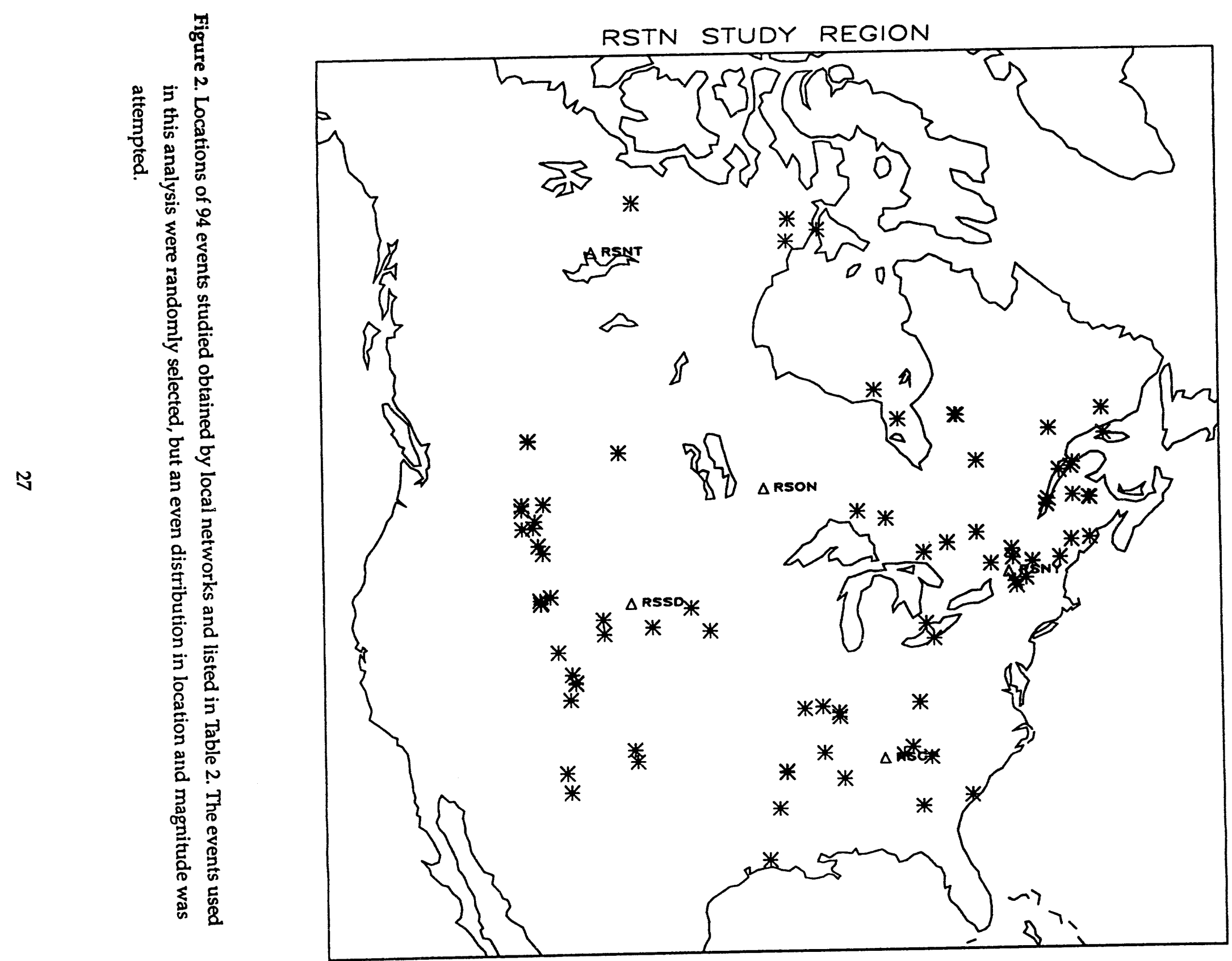

:/rstn/figures $\quad 1001$. sgf 


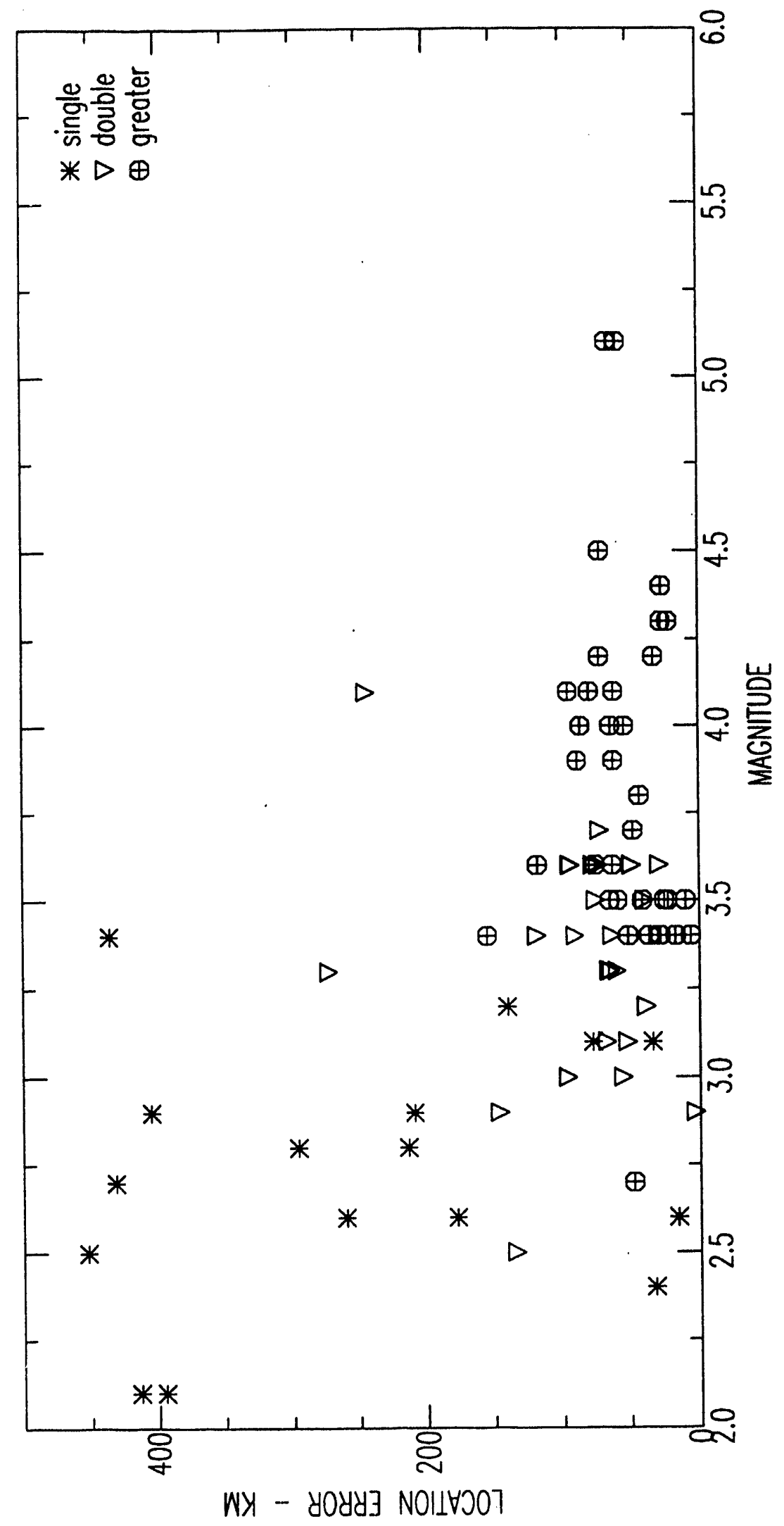

Figure 3.Location error vs. magnitude for events located with the average crustal model. events with location error grater than $500 \mathrm{~km}$ are not shown. Symbols indicatc how many stations were used in the location: single (one), double (two), and greater (three or more). 


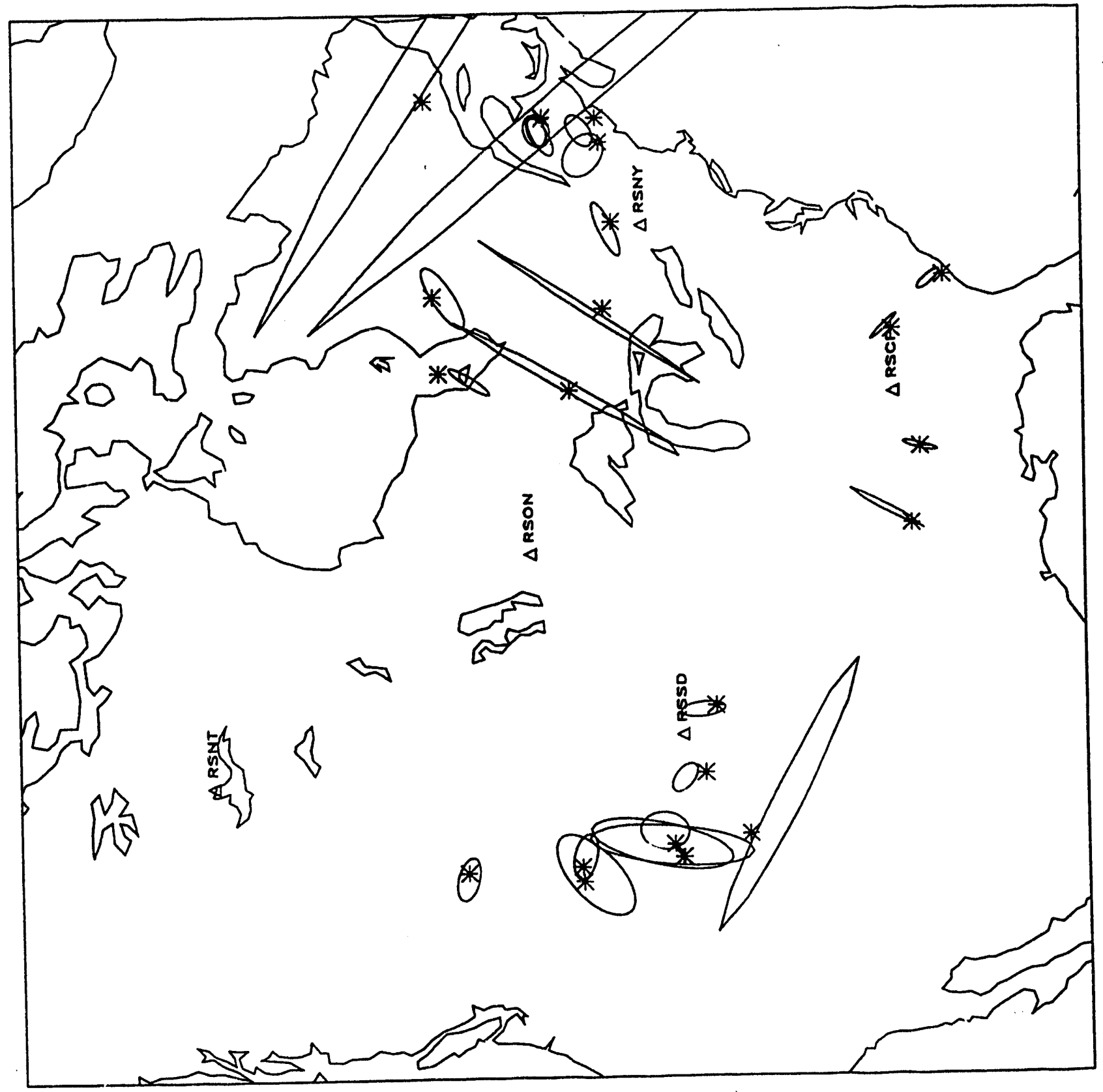

Figure 4. Ninety-five percent confidence error ellipses for events located with two stations and with the average crustal model. Also shown is the location of the events as determined by local networks. Seventeen of the twenty-six error ellipses include the location of the event. 


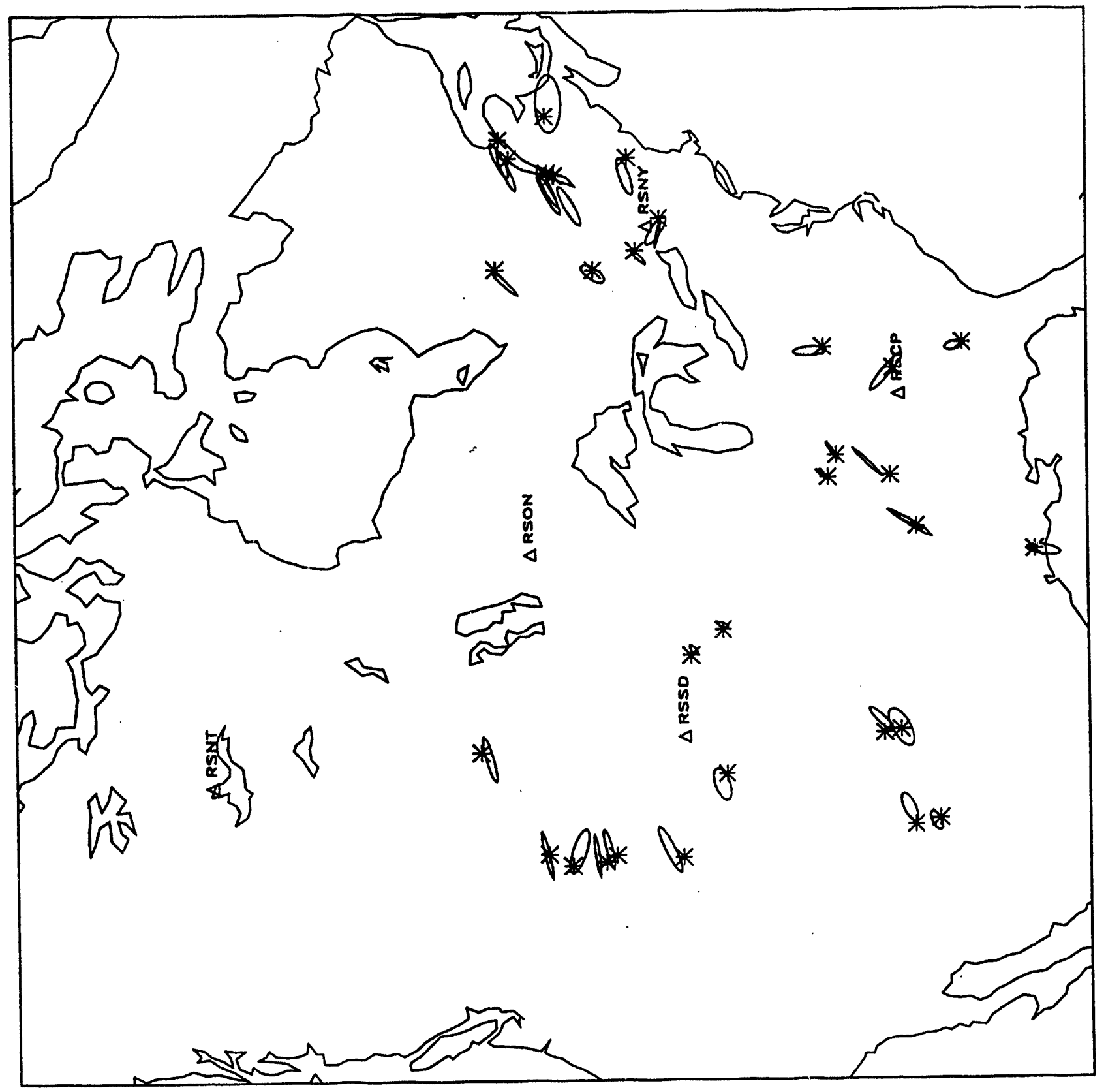

Figure 5. Ninety-five percent error ellipses for events located with three or more stations and with the average crustal model. Also shown is the location of the events as determined by local networks. Sixteen of the thirty-three error ellipses include the location of the event. 


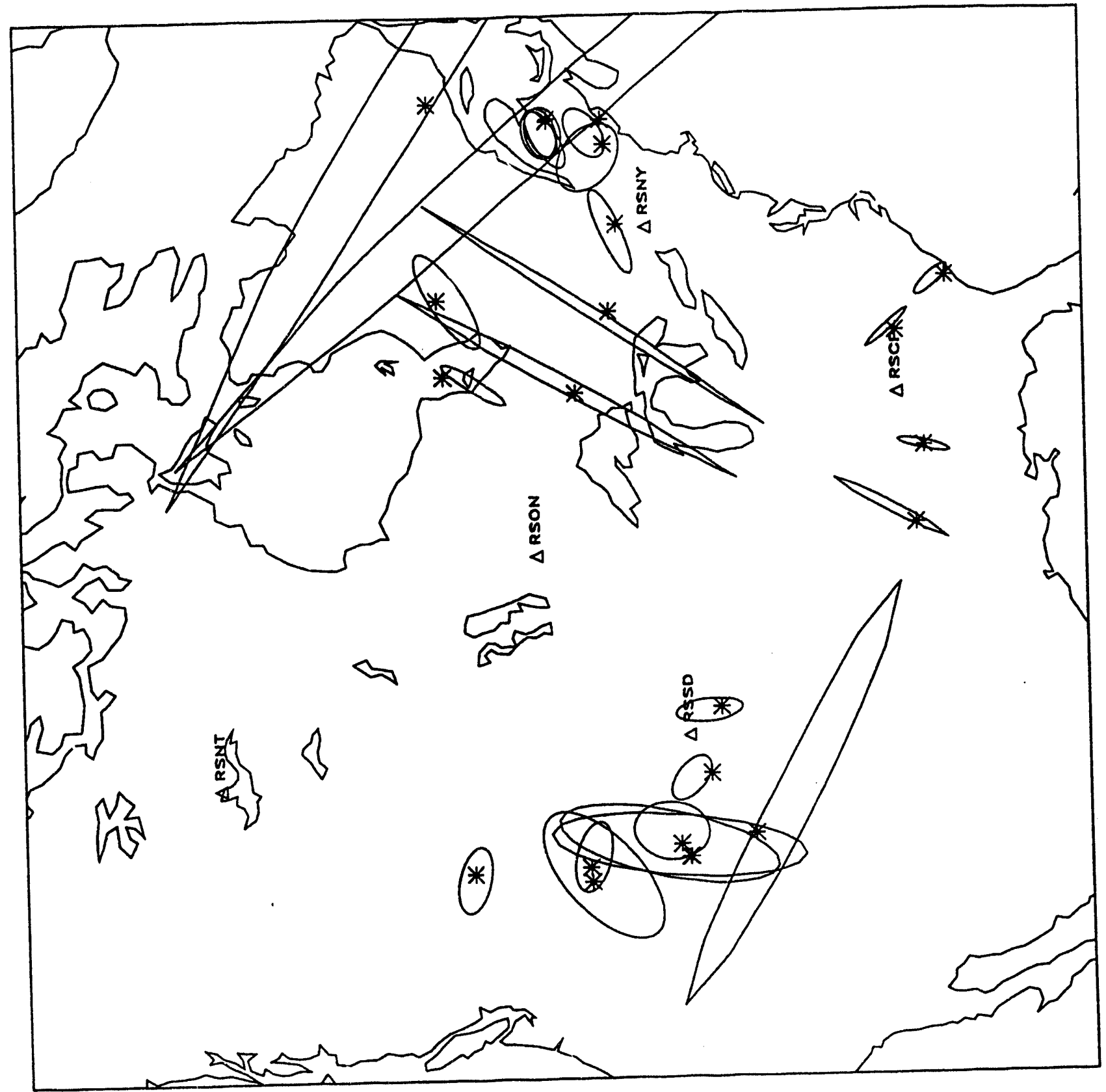

Figure 6. Ninety-nine percent confidence error ellipses for events located with two stations and with the average crustal model. Also shown is the location of the events as determined by local networks. Twenty of the twenty-six error ellipses include the location of the event. 


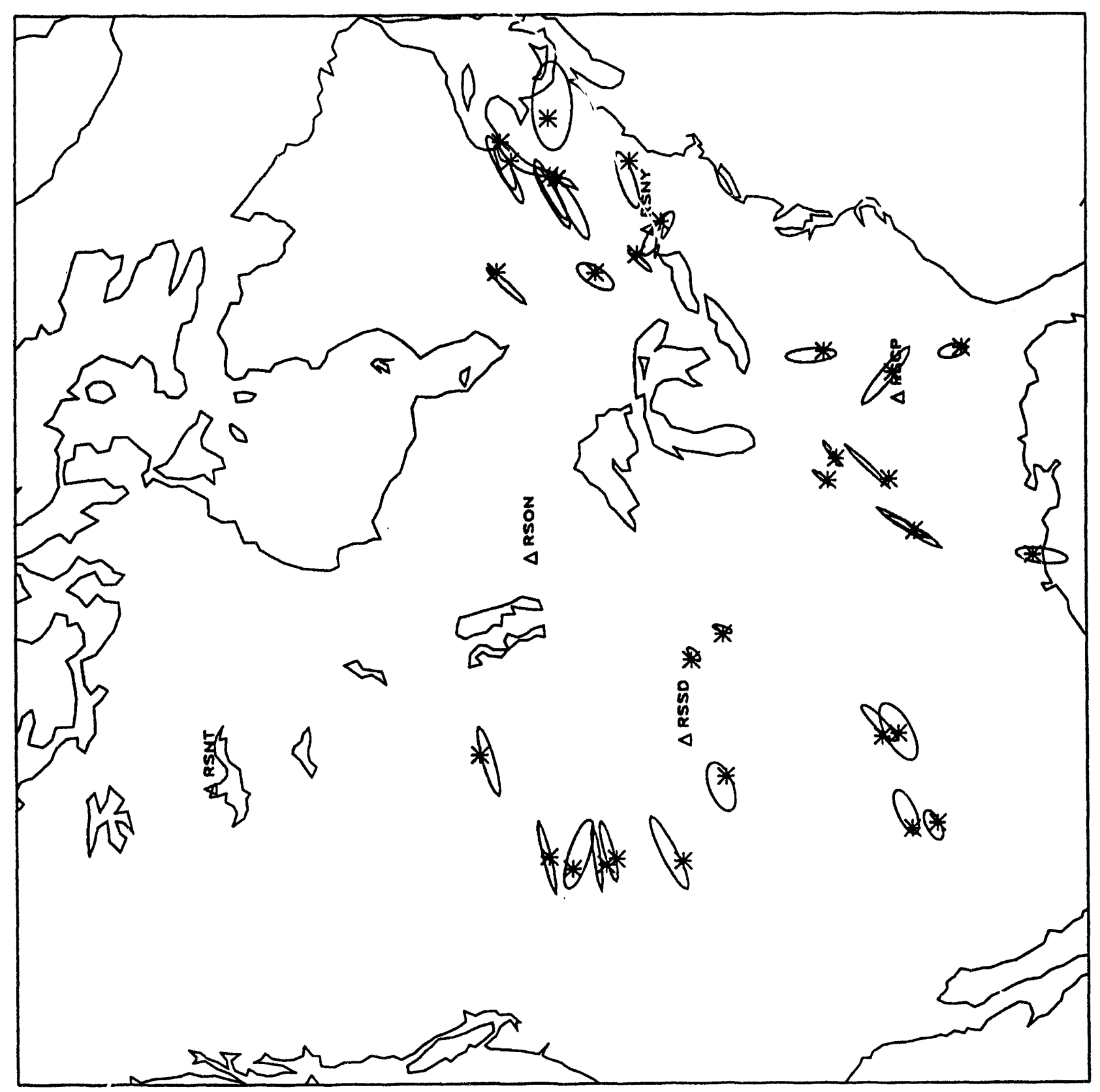

Figure 7. Ninety-nine percent error ellipses for events located with three or more stations and with the average crustal model. Also shown is the location of the events as determined by local networks. Twenty-four of the thirty-three error ellipses include the location of the event. 


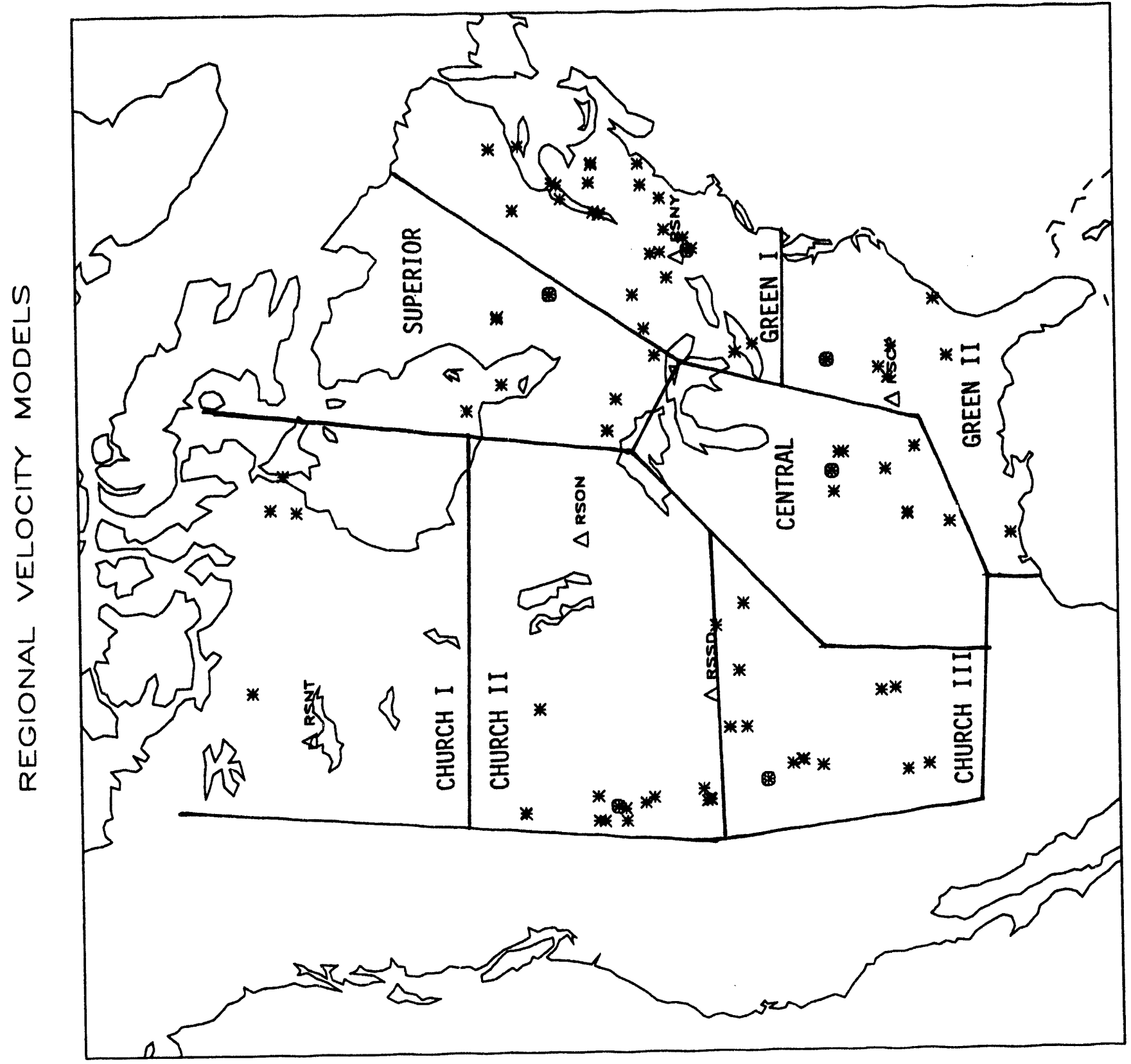

Figure 8. The study area divided into regions that roughly correspond to the provinces shown in Figure 1, along with event locations listed in Table 2 and station locations. The Greenville and Churchill provinces have been further divided into sub-regions for purposes of using master events in location calculations. Events located within each region, or subregion, are located with the same velocity model and station corrections. The velocity models for the provinces are listed in Table 9, and the station corrections are listed in Table 10. Master events used to obtain station corrections are shown by a different symbol. No master event was used for the Church I region. 


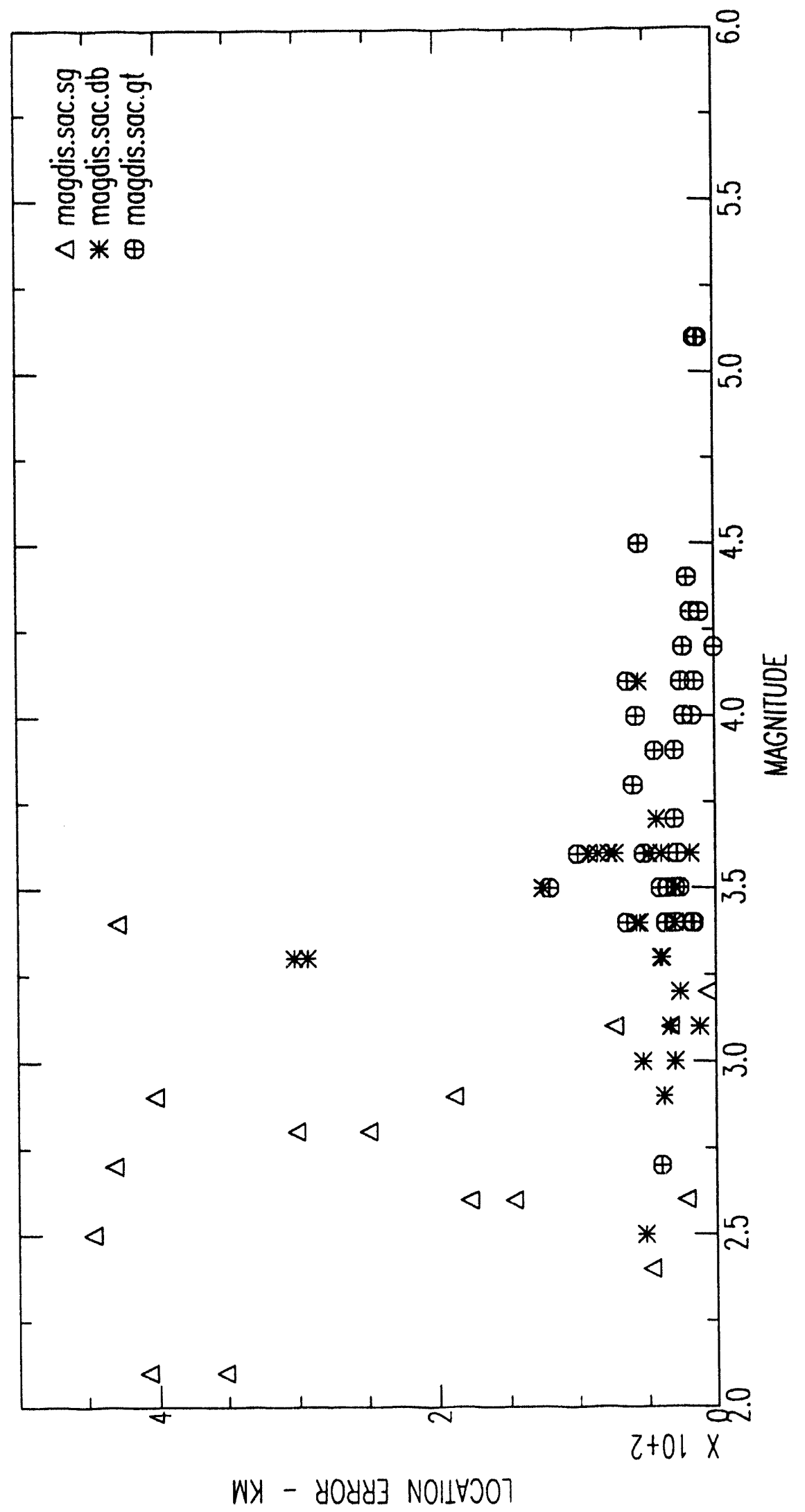

Figure 9. Location error as a function of magnitude. 


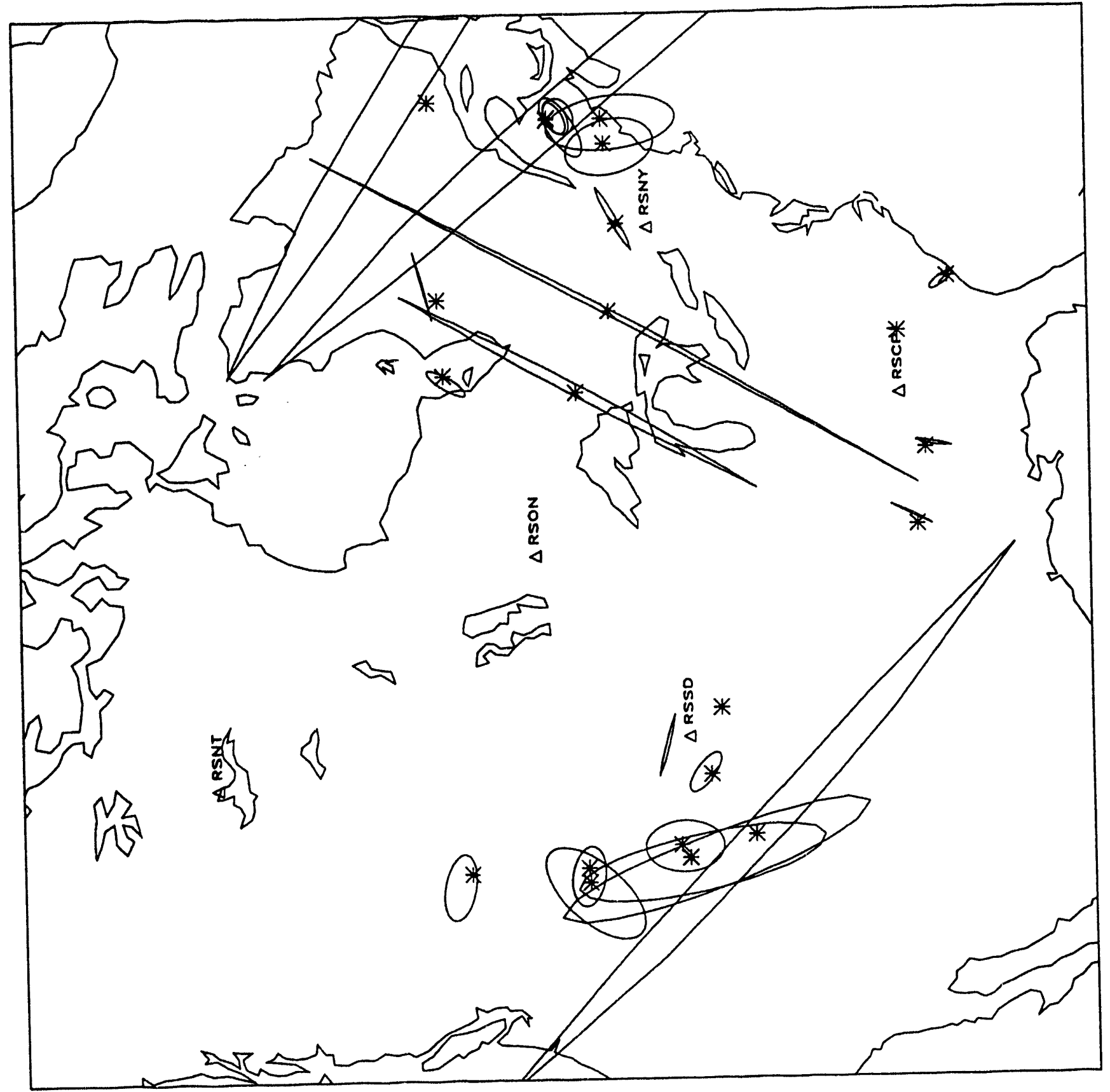

Figure 10. Location error ellipses with $95 \%$ confidence and the actual location of the event as listed in Table 2 , and for events located with two stations. Twenty of the 26 ellipses include the actual location of the event. 


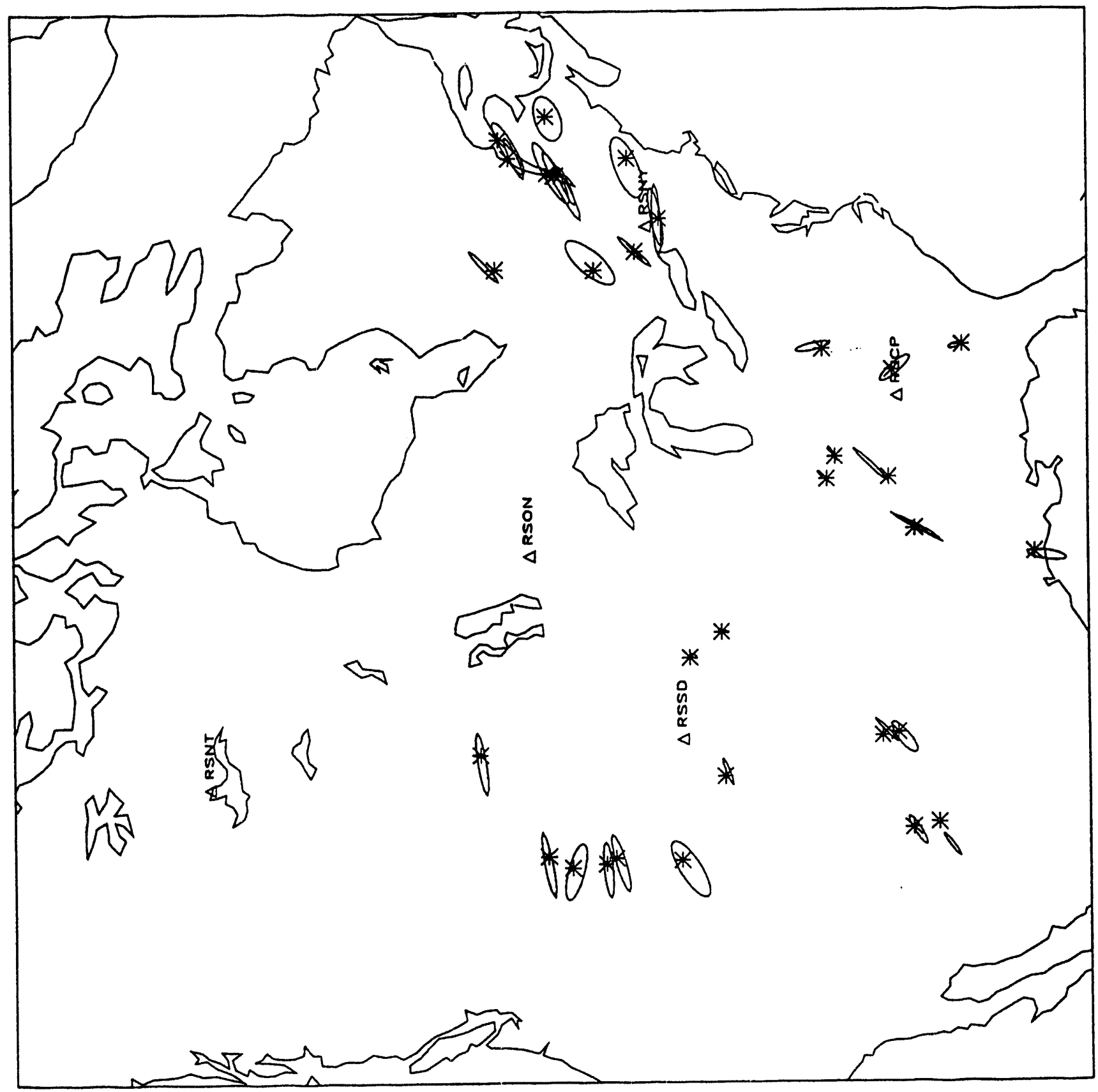

Figure 11.Location error ellipses with $95 \%$ confidence and the actual location of the event as listed in Table 2 , and for events located with three or more stations. Twenty-eight of the 33 ellipses include the actual location of the event. 


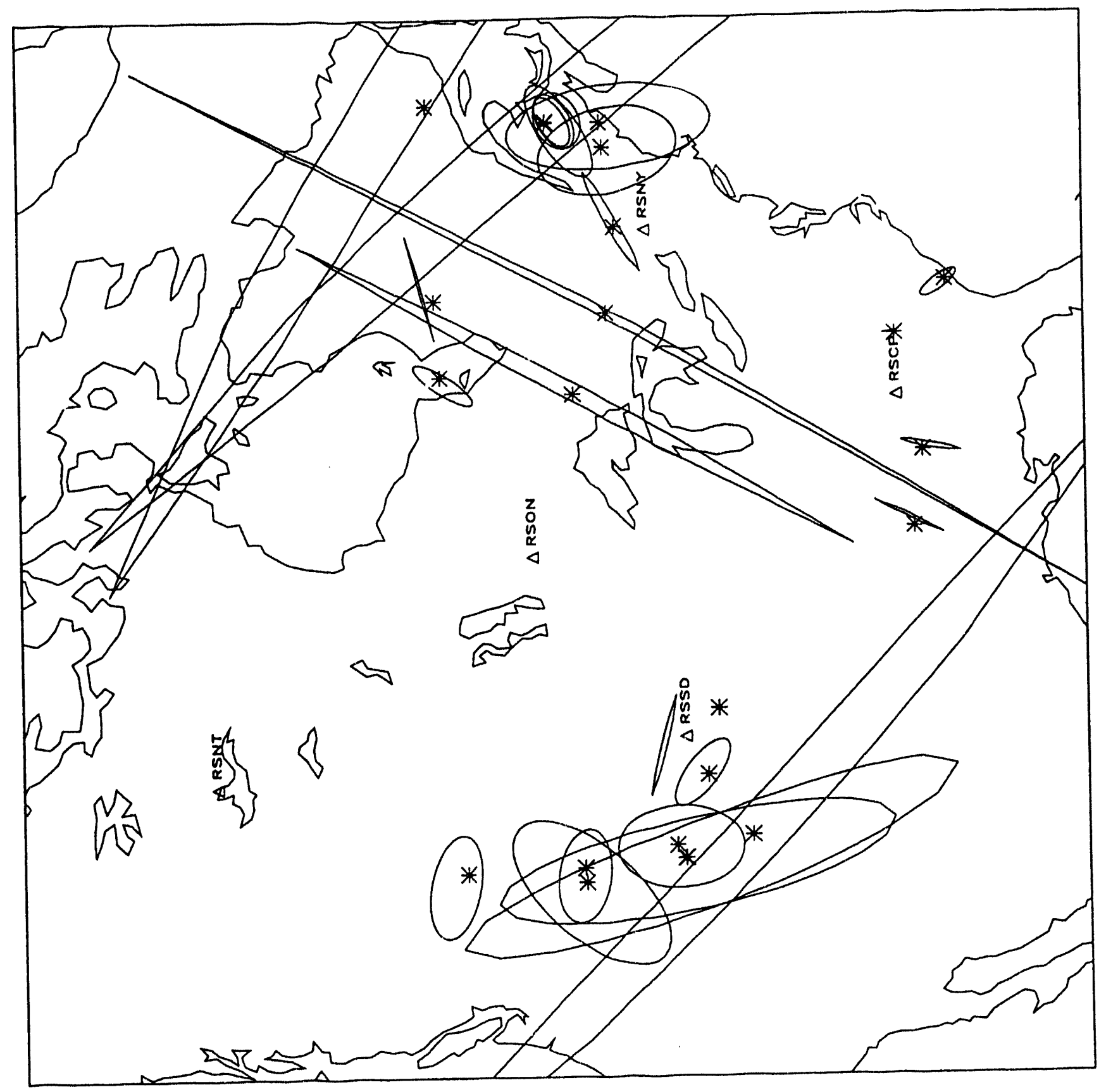

Figure 12. Location error ellipses with $99 \%$ confidence and the actual location of the event as listed in Table 2 , and for events located with two stations. Twenty-one of the 26 ellipses include the actual location of the event. 


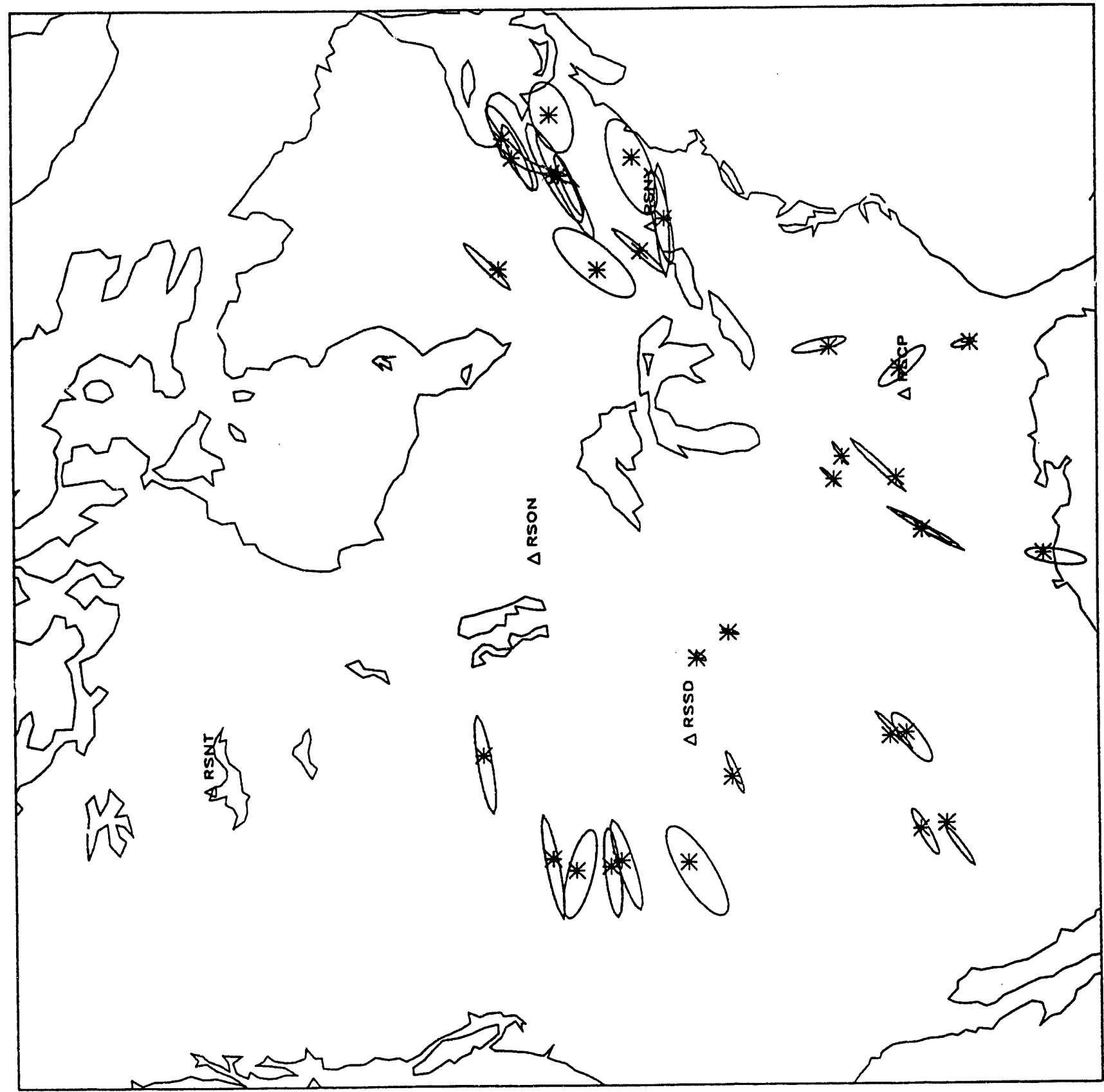

Figure 13.Location error ellipses with $99 \%$ confidence and the actual location of the event as listed in Table 2 , and for events located with three or more stations. Thirty of the 33 ellipses include the actual location of the event. 


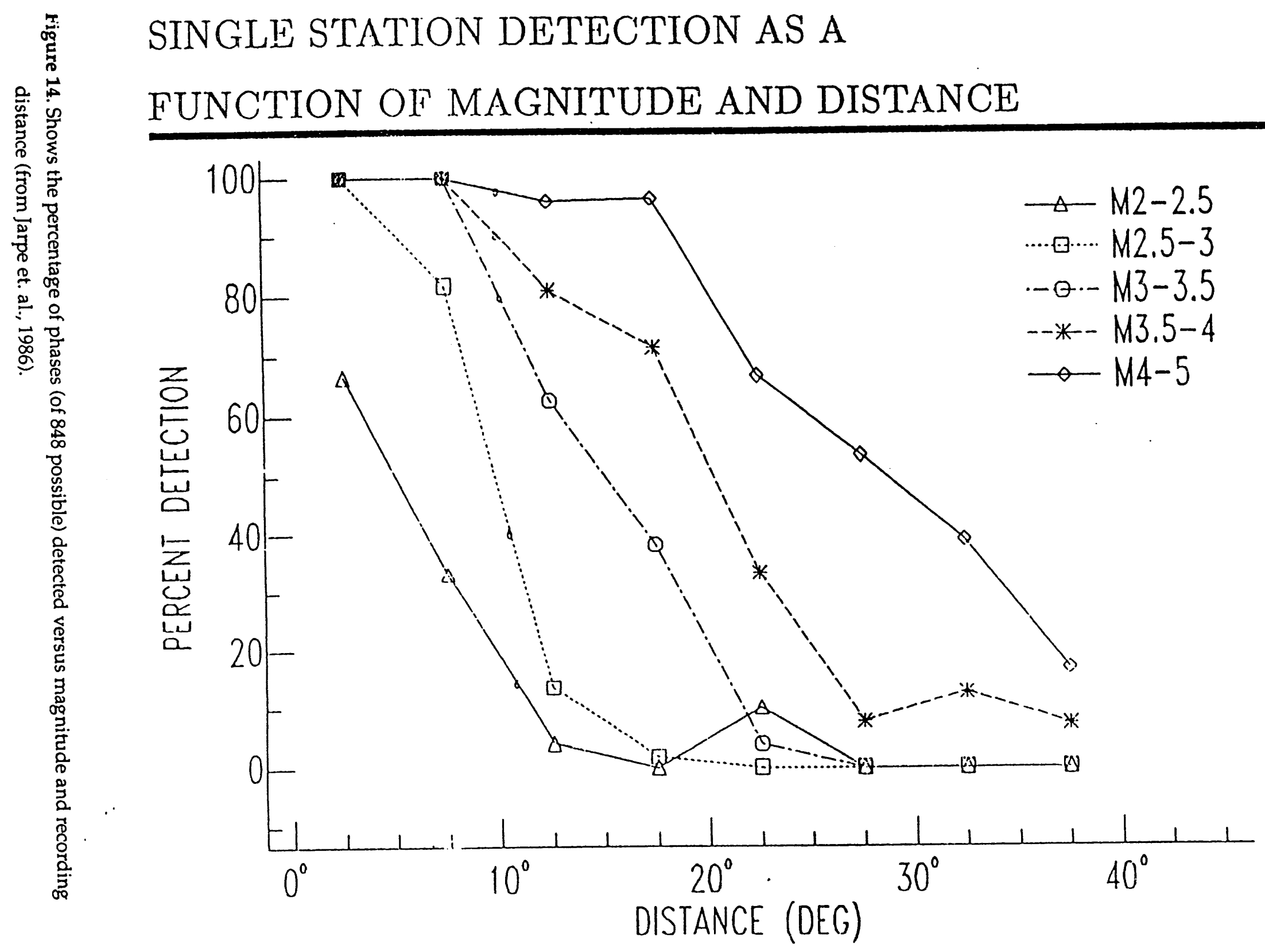




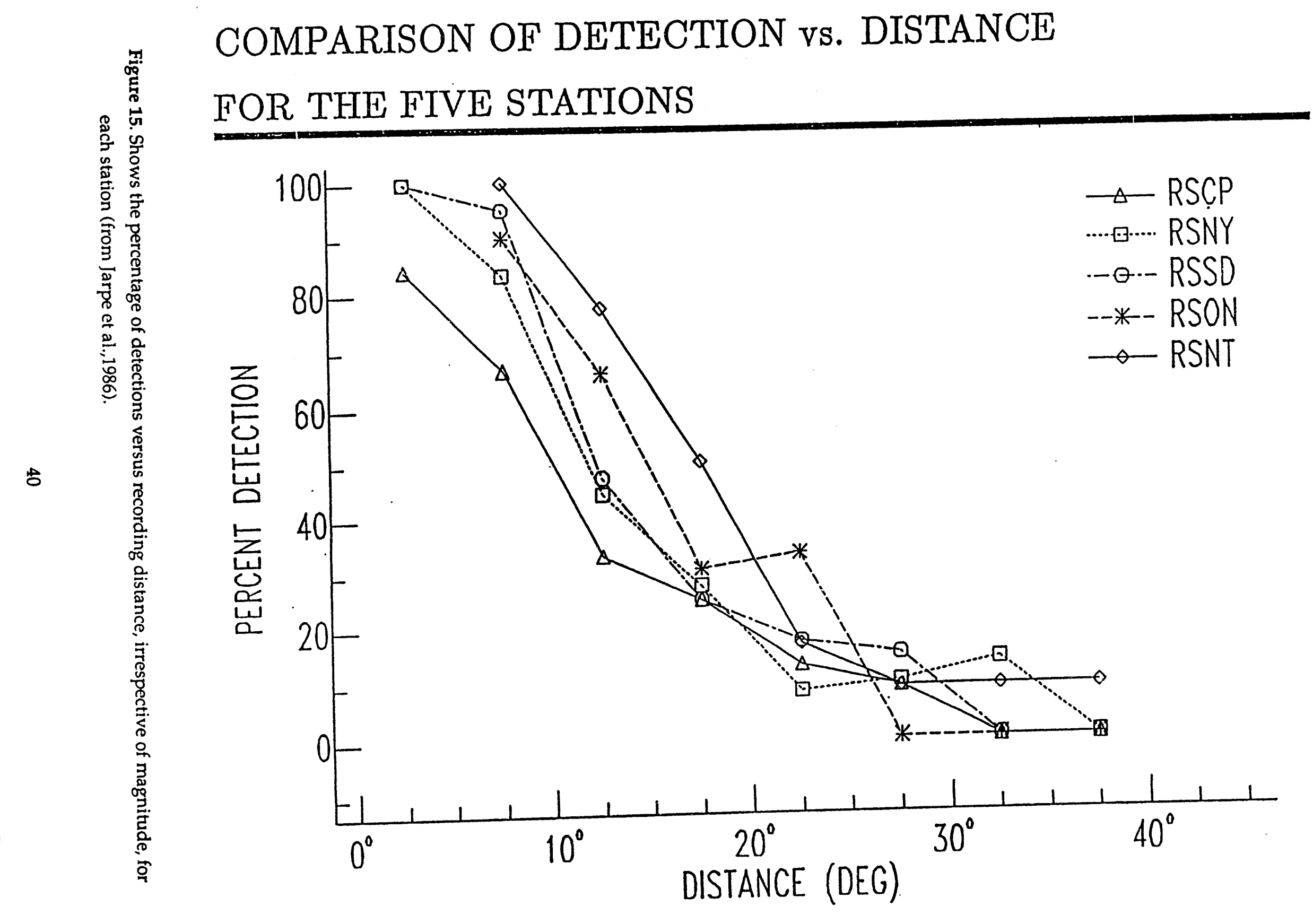




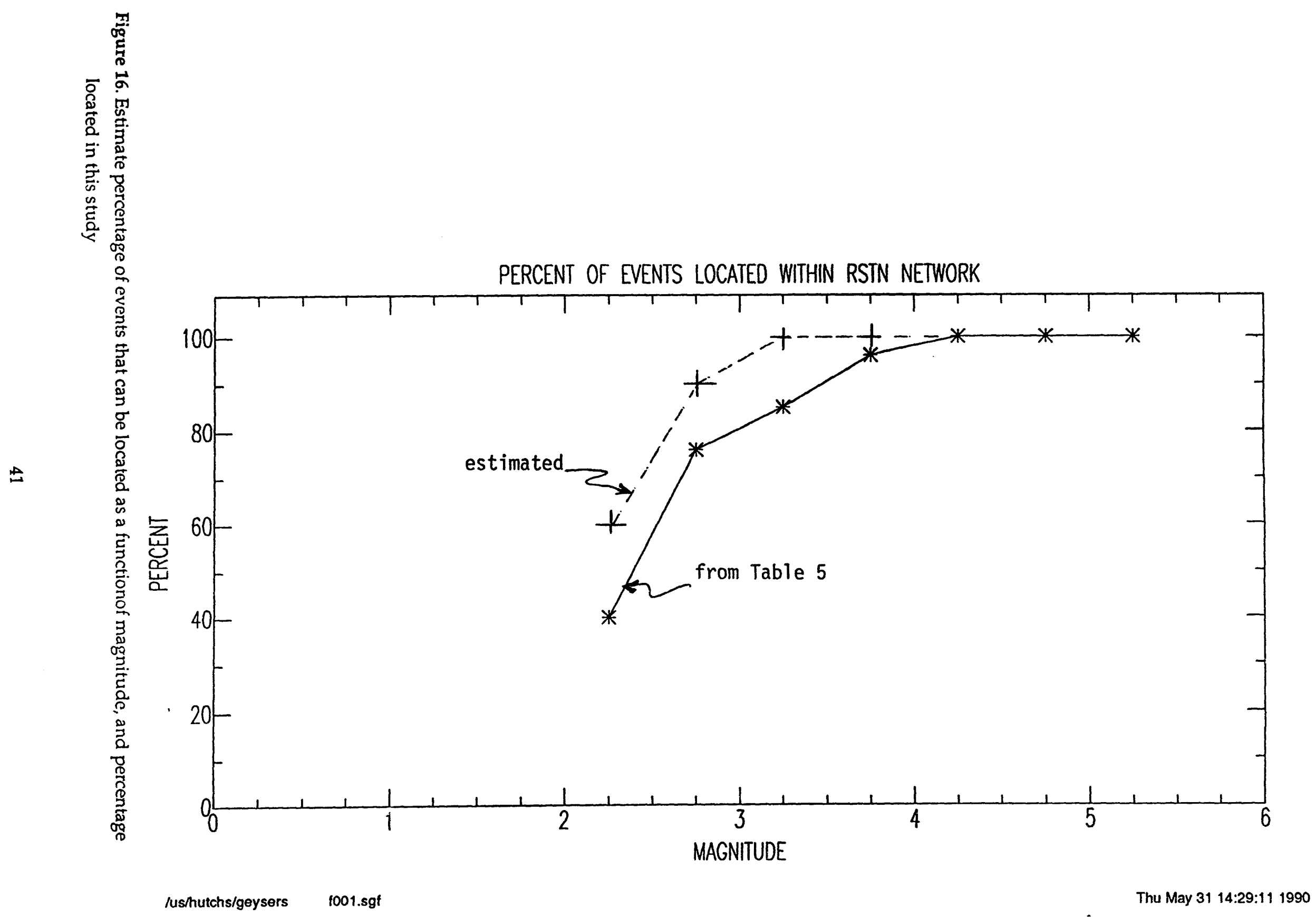


Appendix A

Phase Arrival Times and Backazimuths 


\section{phases.central}

rscp pn 382268231829.94 rscp pg 182268231846.30 rscp sn 082268231928.90 rsny la 282268232541.20 rson pn 282268232042.50 rson sn 282268232326.90 rson lg 182268232519.40 rssd pn 182268232003.40 rssd sn 182268232222.70 rssd 19 182268232350.90

rscp pn 282325163646.52 rscp pg 182325163701.13 rsce sn 082325163752.90 rson sn 382325164148.00 rson 38232516433720 rssd on 382325163826.30 rssd sn 382325164043.20 rssd Iq 182325164210.30

rscp pn $0830190>3202.25$ rscp P9 083019023221.30 rscp sn 183019023303.80 rscp lg 083019023326.90 rsny la 3 s 83019023909.20 rsnt 19483019023909.20 $r$ rson sn 183019023700.70 rson ts 283019023847.30 rssi on 183019023342.70 rssd sn 183019023556.40 rssd lg $0 \quad 83019023725.20$

rscp pq 283036130901.8 rsco lg 183036130934. rssd lg 383036131622.7

rscp pn 383054085220.25 rscp p9 083054085225.75 rscp to 083054085312.90 rsny lo 283054085820.80 rson pn 283051085456.20 $r \operatorname{son} p g 283054085619.60$ rson sn 283054085732.70 rson iq 38305408596.90 rssd sn 28305408574.40 rssd lg 183054085837.20

rscp pn 083135051730.35 rscp pg 083135051741.69 rsep sn 083135051821.20 rsep 190 o3135051831.20 sny sn 083135052131.20 rsny ig 083135052246.20 rson pn 083135051914.80 rson sn 083135052123.10 rson 19 083135052245.10 rssd pn 083135051911.70 rssd sn 083135052118.80 35052237.00

rscp pg 383172135944.2

$\begin{array}{llll}\text { rscp sn } 3 & 83172140025.8 & 278.5\end{array}$ rscp Ig 283172140047.6

rsce sn 383173040547.7

rssd ta 483173040940.0

rsny sn $383343055046.20 \quad 0.0$

rscp pg $084108044546.09 \quad 0.0$ rscp sn $084108044621.83 \quad 0.0$

rscp Ig O $84108044629.60 \quad 0.0$

rsny 19 $384108045053.10 \quad 0.0$

rson sn $184108044959.60 \quad 0.0$

rson $193384108045125.80 \quad 0.0$

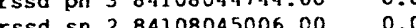

rssd to 184108045130.60 
hutchs

(3)

phases.church1

rsnt pn $382296055150.10 \quad 0.0$

rsnt sn $382296055338.60 \quad 356.0$

rsnt pn $082360081413.2 \quad 064.0$

$\begin{array}{lll}\text { rsnt pg } 4 & 82360081427.8 & 049.0 \\ \text { rst } & & \end{array}$

rsnt pn 382360084932.0

rsnt sn $282360085129.2 \quad 144.0$

rsnt Ig 382360085247.2

rssd Ig 4830821147 


\section{hutchs}

\section{phases.church2}

pn $282296123026.50 \quad 0.0$ rssd pg $182296123050.10 \quad 332.0$ rssd sn $282296123138.40 \quad 0.0$ $\begin{array}{llrr}\text { rssd Ig } & 0 & 82296123212.00 & 276.0 \\ \text { rson pn } 3 & 82296123147.00 & 0.0\end{array}$ rson sn $282296123407.40 \quad 0.0$ rson lg $2 \quad 32296123531.70 \quad 196.0$

rsnt pn 482330200549.50 40.00 rson lg $282330200845.90 \quad 0.0$ rssd pn 282330200333.610 .0 rssd pg 182330200356.00 $\begin{array}{llll}\text { rssd sn } 1 & 82330200438.70 & 0.0 \\ \text { rssd 1g } & 0 & 82330200510.10 & 0.0\end{array}$

rsnt pn $183006023741.6 \quad 144.0$

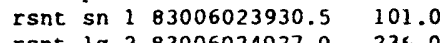
rsnt 19 $283006024027.0 \quad 236.0$ rson sn $083006023833.1 \quad 288.0$ rssd pg $283006023733.5 \quad 389.0$ rssd pg $283006023733.5 \quad 389.0$

rsnt pn 283047062522.10

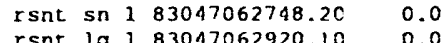
rson pn $183047062500.50 \quad 0.0$ $r$ son sn C 83047062108.90 0.0 rson Ig $083047062834.60 \quad 0.0$ rssd pn $383047062356.30 \quad 0.0$ rssd pg 3 83047062419.40 0.0

rssd on $3 \quad 83 \quad 47071532.54$ rssa po $0 \quad 83 \quad 47071553.80$ rsso 1900834757171.40 rson on $2 \quad 83 \quad 4707: 7 \quad 8.90$ rson sn 28347071942.40 rson lg 18347072045.70 rsit on 2834707182.20 rsnt lo 283347072245.80

rscp pn 28376013128.20 76073757.50 rson pn 08376072857.90 rson pg $283 \quad 76072955.20$ rson sn $083 \quad 76073118.80$ rson 1908376073237.40 rssd pn $083 \quad 76072739.50$ rssd pg $0833 \quad 76072809.00$ rssd lg $083 \quad 76072925.20$

rson pn $383081045810.60 \quad 0.0$ rson sn $383081050030.00 \quad 304.0$ rson lg $383081050208.30 \quad 288.7$

rssd pg $383082014358.9 \quad 325.7$ rson pn $283313135611.80 \quad 0.0$

rson $19383313135956.60 \quad 0.0$ rssd pn $0 \quad 83313135417.27 \quad 288.0$ $\begin{array}{llll}\text { rssd pg } 0 & 83313135425.03 & 276.0 \\ \text { rssd lg } 0 & 83313135520.00 & 308.0\end{array}$ rscp pn $483354225708.90 \quad 0.0$ rsnt pn $183354225651.50 \quad 0.0$ rson 0083354225538.700 .0 rssd pn 083354225338.35 rssd po 083354225352.82 rssd la $083354225454.00 \quad 0.0$

rson pn $2833542325 \quad 9.20 \quad 0.0$ rson 1g $48335423294.40 \quad 0.0$ rssd pn $28335423238.27 \quad 284.0$ rssd pg $083354232321.48 \quad 276.0$ rssd 190 $033354232423.00 \quad 296.0$ rson pg $383355202940.20 \quad 0.0$ rssd pn $383355202711.40 \quad 324.0$

rson pn $283356185918.40 \quad 196.0$ rssd on $183356185771.07 \quad 224.0$ rssd pg $0 \quad 83356185733.11 \quad 256.0$ rssd Ig O $83356185835.90 \quad 268.0$ $\begin{array}{lllll}\text { rssd pg } & 84084052319.80 & 256.0 \\ \text { rssd } 19 & 1 & 84084052420.80 & 316.0\end{array}$ 


\subsection{6 .105 \\ 11:06.5.}

rscp pn 382287125659.90 rscp If 182287130059.20 rsny If $3 \quad 82287130547.30$ rson on 282287125730.10 rson sn 282287130020.00 rson 1g 1 82287130204.80 rssd pg 082287125619.50 rssd sn 082287125719.00 rssd sn 0 82287125719.00
rssd 19 o 82287125809.50

rscp pn 082319030112.20 rscp sn 082319030323.10 rscp la $0 \quad 82319030432.00$ rsny pn 382319030219.10 rsny sn 282319030521.00 rsny 1g 182319030708.60 rson sn 082319030149.90 rson 19082319030236.60 rssd pn 082319025932.74 rssd pq $0 \quad 82319025943.32$ rssd sn 0823190300 rssd lg 082319030044.20

rssd pn $282326101013.40 \quad 236.0$ rssd pg $282326101044.40 \quad 232.0$

rscp pn 283044134844.90 rscp sn 283044135142.60 rscp 19 283044135334.50 404134926.10 rsnt sn 283044135332.40 rsnt la 283044135539.40 rsny lq 383044135630.60 rson th 083049134930.80 rson 10083044135057.30 rssd la $0 \quad 83041134555.26$

rscp pn 38361232736.90 rscp sni 18361233019.70 rscp Ig 28361233236.00 rsny lg $3 \quad 83 \quad 612337 \quad 9.70$ rssd pq $0 \quad 83 \quad 61232626.40$ rssd $19083 \quad 61232840.90$

rscp pn 083063063530.50 rscp sn 083063063754.90 rsny pn 383063063623.10 rsny sn 283063063938.20 rsny lg 183063064121.30 rson pn 08306306347.70 rson sn 083063063529.70 rson lg o 83063063613.10 rssd pn 083063063310.4 rssd pq 083063063318.72 $\begin{array}{lll}\text { rscp pn } 3 & 83093045834.30 \\ \text { rscp ig } & 3 & 83093050233.80\end{array}$ hutchs

\section{phases.church 3}

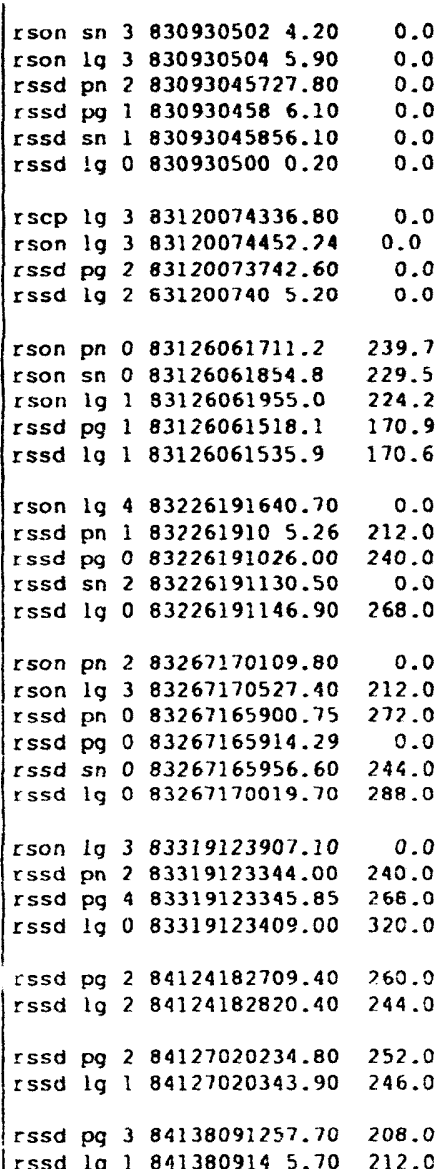


rsny pn 382276043353.40 rsny Sn 182276043555.90 rson sn 482276043930.30 rson 19482276044125.70

rsny pn 082299153301.21 rsny sn 182299153407.80 rsny lg 082299153441.10 rson 1g 482299154052.80

rscp 1g 282338161712.70 rsnt pn 382338161456.10 rsnt sn 282338162013.40 rsny pn 082338160934.61 rsny pg 0 23338160942. rsny lg 082338161038.60 rson pn 382338161210.40 $r$ son pg 182338161308.60 rson sn 182338161458.10 rson 1g 082338161627.50 rssd la 382338162058.90 rson pn 382351055107.8 $r$ son sn 382351055354.4

rsny pg 282357070801.9 rsny sn 182357070859.0

esny pn 183010213224.3 rsny sn 183010213542. rson 19 183010213644.2

$\begin{array}{llll}\text { rsny } & \text { sn } & 3 & 83017082255.9 \\ \text { rsny } & \text { Iq } & 3 & 83017082322.9\end{array}$

rscp pn $383 \quad 19174013.40$ $\begin{array}{lrl}\operatorname{rsc} 19 & 283 & 819174538.20\end{array}$ rsne pn 3 s 3319174736.80 rsnt sn 3 83 19174734.20 rsny pn 0 83 19173841.30 rsny lo $083 \quad 19173916.20$ rson pn $383 \quad 19173949.30$ rson sn 183319174257.70 rson 1g $083 \quad 19174434.30$

rsny pn 183020141840.7 rsny sn 283020141939.3 rsny 1g 283020142008.2

rsnt Ig 383042160147.40 rsny pn 183042154825.14 rsny 1g 08304215504.60 rson pn 383042155042.70 rson sn 383042155335.10 rson 1g 283042155523.25

rsny pn 283133172730.28
22.0 corrected from 005.0 66.0 correcter from 009.0 65.0

\section{9}

0.0

75.5

0.0

0.0

0.0

0.0

0.0

0.0

0.0

0.0

0.0

078.0

277.0 corrected from 260.0 278.0 corrected from 261.0

319.4 corrected from 302.4

308.4

100.0

125.0 corrected from 108.0

0.0
0.0
0.0
0.0
0.0
0.0
0.0
0.0
0.0
0.0

054.0 corrected from 036.9 051.0 corrected from 034.0 from 039.9

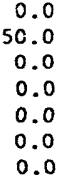

67.7 corrected from 50.7

\section{phases.green 1}

rsny pg 283133172810.60 rsay sn 283133172836.90 rson lg 283133173526.20

rscp pn 283133234508.40 rsCP Pg 183133234614.60 rscp If 183133235011.90 rsnt pn 383133234745.50 rsnt sn 383133235325.30 rsny pn 083133234225.14 rsny sn o 83133234332.20 rson pn 283133234506.30 rson sn 383133234817.90 rson 19 383133235011.50 rssd on 383133234657.60 rssd sn 283133235144.70 rssd la 283133235432.00

rscp pn 383136020551.00 rsCp pq 483136020723.90 rscp lg 283136021109.50 rsrit pn 483136020819.60 rsnt sn 383136021322.30 rsny on 283136020304.30 rsny sn 283136020355.20 rsny lg 083136020413.00 rson sn 283136020822.70 rson 1g 083136021001.40 rssd sn 383136021155 rssu 1g 283136021425.70

rscp pn 283149054923.90 $r s c p$ pg 483149054951.80 rscp sn 38314905526.00 rscp 190 83149055323.30 rsny 3383149054636.87 rsny po 0 o3149054641.54 rsny so 333149054705.36 rsny ta 083149054720.65 rson on 483149054847.70 rson ig 083149055426.70 rssd on 383149055131.40 rssd sn 383149055611 rssu 1g 283149055822.40

rscp Ig 383153063850.50 rsny pn 283153063126.30 rsny sn 383153063211.90 rsny ig 0 o3153063224.40 rson sn 283153063651.70

rsny pn 183162134926.74 rsny sn 083162135033.20 rsny 19 $0 \quad 831621351 \quad 4.80$ $r$ son ig 283162135723.50 rsny pig 083167163720.5

0.0 46.3 94.5 0.0 0.0 0.0 0.0 0.0 0.0 0.0 0.0 0.0 0.0 0.0 0.0 corrected from 53.2

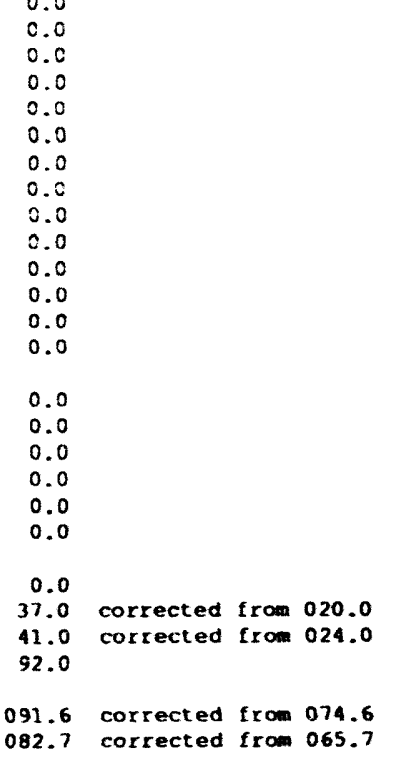




\section{hutchs}

\section{phases.green 1}

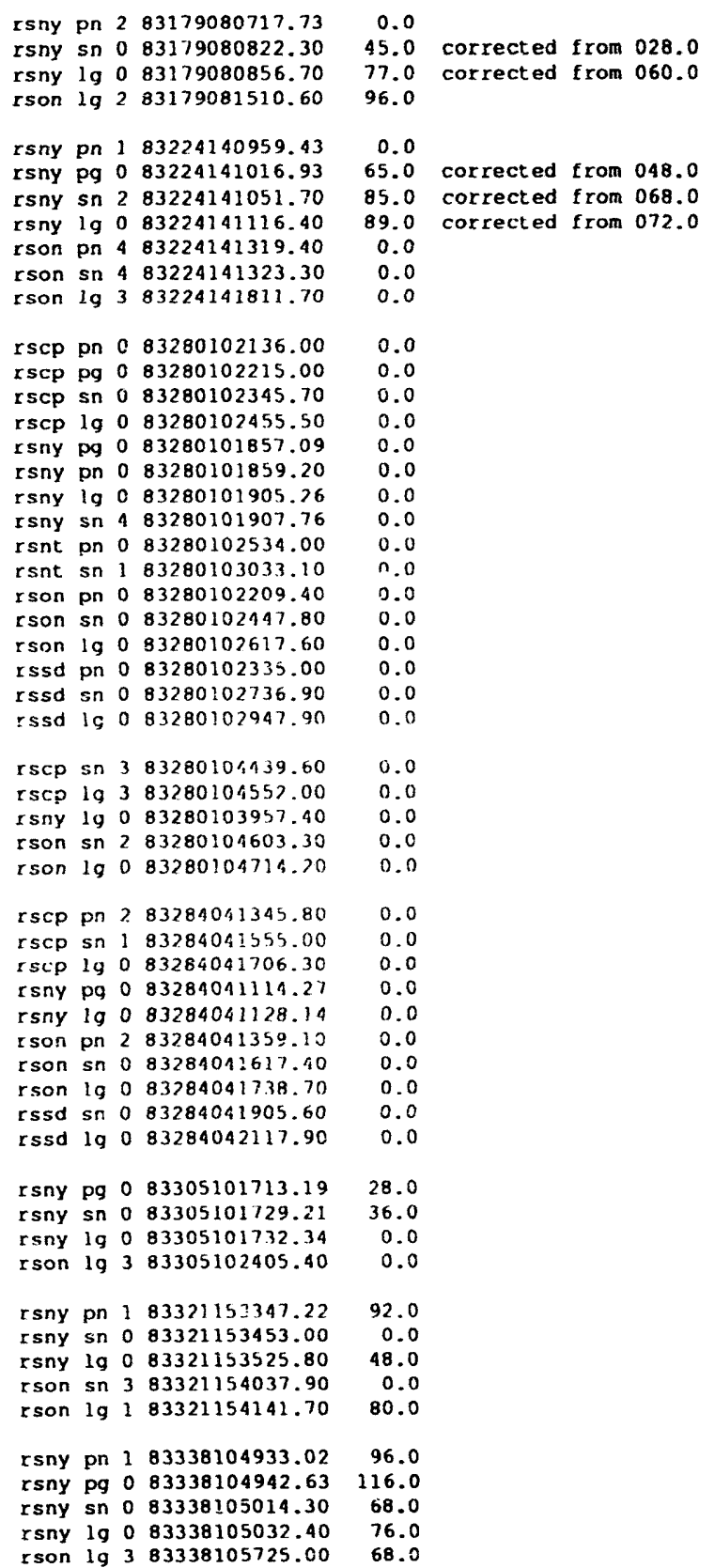




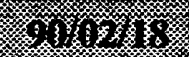

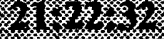

rscp pg 082267221936.48 rscp 19 082267221950.83

rsny sn 32267222157.50

rsny lo 382262222514.70

rssd lg 382267222820.00

rscp pg 083026140844.39 rssd Ig 283026141758.80

rscp pg 183027221004.4 Iscp 1g 183027221025.9

rscp pg $183084024755.5 \quad 092.8$ $\begin{array}{lllll}\text { rscp lg } & 2 & 83084024829.3 & 143.6 & \\ \text { rsny } & \text { lg } & 83084025243.7 & 217.2 & \text { corrected from } 200.2\end{array}$

rsny pn 183229140517.70 rsny sn 183229140651.10
rsny 19183229140740.10 $r$ son pn 283229140635.10 rson sn 283229140907.60 rson 1g 183229141044.10 rssd sn 283229141015.70 rssd 1928 . 28

$x \operatorname{scp}$ on 083289194253.50 rscp sn 083289194425.20 rson on 28328919453190 rssd pn 183289194410.50 rssd sn 183289194734.40 rssd 1g 283289194922.20

rscp pn $183310090337.30 \quad 140.0$

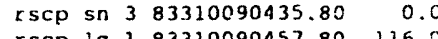
rscp 19 $183310090457.80 \quad 116.0$ rsny lg $383310090842.80 \quad 0.0$

\section{hutchs}

phases.green2 


\section{Appendix B}

\section{Partial listing of REGLOC:}

program to locate earthquakes with sparce data 
purpose: event location program ut $111 \mathrm{zing}$ both phase fone or more onset $s$ and back-azimuth

author: Lawrence Hutchings, LLNL

\section{the program requires 2 input files: \\ "phases" and "stations"}

the program produces 2 out put flles:

and "locat ion"

proqram regloc

include 'regloc. cmn

data $p n, s n, p g, s g, l g, b z /$ pn', $^{\prime} s n^{\prime},,^{\prime p g} g^{\prime}, s^{\prime}, g^{\prime} l g^{\prime},{ }^{\prime} b z ' /$

data blank $\%$,

c The file called "location" contalns the output

it is written into from the main and 2 different subrout ines: "locat ion" and "output

open (12, file ${ }^{\prime}$ location')

option to calculate travel time residuals from a fixed location. fxt $=1.0$

for fixed location, read location from file ' $\mathrm{fxt}$. $\mathrm{loc}^{\prime}$.

$$
f \times t=0.0
$$$$
\text { If ( fxt.eq, } 2,0) \text { then }
$$

read $(15, *)$ if $y r$ ifdy, fhs, ifmin, fsec, flat, tlon, fh endif

c 1 jh

convention on latitude and longitude

c latitue positive for north

congitude positive for cast

Number of location variables (lat, long, origin-time, dept $h$ ) $=n p$

(for np=3, no itcration with depth; begins with 4 , (for np $=3$, no it
is changed to 3 )

is $p=4$

$\mathrm{p} 12=6.28318530717958$

$\mathrm{p} 1=3.141592654$

$\mathrm{dr}=\mathrm{pi} 2 / 360$.

constant to convert to ellipticity:

$g c=(1 .-1 . / 297) * *$.

ia $=$ the order of $g(g$ is a constant in inversion routine

$c$ if $n p=4,1 a=10$

$c$ if $n p=3, i a=6$

$\quad 1 a=10$

c

c To constrain depth to given value, idc $=1$

c To make depth to be a free parameter, idc $=0$

$c$ idc is read from an input file

c For pure least squares, set damp $=0 \quad\{=1$ otherwise $)$

catlog: reads in a catalog of previously located events from c a file called "summary"

call catlog velsta: reads in stations and velocity model info from a flle

called "stations"

call velsta

if (idc.eq.0) go to 10

$\mathrm{np}=3$

c rearriv: reads in phase information

c from the flle "phases"

io $1 \mathrm{rtn}=0$

call rearriv

if (irtn .eq. 401 ) goto 401

fif (irtn eq. 402 ) goto 402

c locate: does the location and writes to a flle called "location"

call locate

cslmtx: Also writes into "location", the covarlance, data resolution and model resolution matricies, and other information.

call rslmtx

c output: Also writes into "location" the final solution and error ellipse information call output

c sumfile: updates the file called "summary" to include the new location a running catalog

go to 10

401 write $(12,1001)$

write $(6,1001)$

Cole to determine a solution'l

go to 10

102 stop 


\section{8:11:14:}

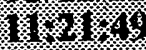

subroutine rsimtx

include' regloc.cmn'

real $\mathrm{np1}$

$g$ (ia) constants of inversion matrix

la order of 9

np number of parameters solved for

c approach to calculating sigma for error ellipses:

$c$ use BAZ for sigmad only if location is with 2 stations

(

c estimate true variance, first prior varlance

sigmap $=0.0$

$\mathrm{np} 1=0.0$

do 101 i $=1$, nosta

do $201 j=1$, nopha

if (awt (j) .eq. 0.0 ) go to 201

if (stat (j).ne.sta (i)) go lo 201

if (pha (j) .ne.pn) go to 202

$n p l=n p l+$ float (nrpn (i)

sigmap = sigmap + rpn (i)

go to 201

202 if (pha (j).ne.pg) go to 203 $n p 1=n p 1+$ float $(n r p g(i)$ slgmap $=$ slgmap $+\mathrm{rpg}(1)$

in to 201

203 pha (j). ne.sn) go to 204 $n p l=n p l+t$ loat $(n r s n(i)$ sigmap $=$
go to 201

204 if (pha (j) - ne. sg) go co 205 npl - npl + iloat (nrsg(i)) sigmap - sigmap + rsq(i) go to 201

205 if (pha (j).ne.1g) go to 206 $n p l=n p l+f l o a t(n r l g(i))$ sigmap = sigmap + nrlg(i) go to 201

206 If (pha(j).ne.bz) write $(12,110)$ pha(j)

110 format ('phase 'ar, mlstdent aakm $=\operatorname{acos}(\sin (r t)$

$8 * \cos (r) a m-r l(1))$

c remember wt $=0.1$ for baz for non-algebraic solut lons

$c$ and baz distance weighted

if (1rtn. ne.404) then

$\left(\frac{1}{2} * * 2 / v 1 g * * 2\right) * \operatorname{rbz}(1) * d r * * 2$

$n p 1=n p 1+w t * f l o a t(n r b z(1))$

end if

If (irt.n.eq. 404) then

$0,1 *(d i s t t * * 2 / v 1 g * * 2) * \mathrm{rbz}(1) * \mathrm{dr} * * 2$

$\mathrm{np1}=\mathrm{np1}+$ float (nrbz (1))

endif

201 continue

sigmap $=$ sigmap/(np1 - float $(n p))$

now estimate true variance hutchs

rslmtx.f

c

if (npl+df.1t.7.) then

sigmap $=12.0$

npl $=4.0$

if (df.le.1) then

$\mathrm{df}=1.0$

sigmad $=\mathrm{float}(\mathrm{df}) * 3.0$

endif

endif

sigma $=(n p l-f l o d$
ssigmad/ (npl+df)

c compute resolution matrices

c cote $G$ in Menke 11984, Geophysical c data analysis: discrete inverse theory). We actually use his $\mathrm{G}-\mathrm{g}$ here, c but since G-g is a symetric matrix, the same relations hold.

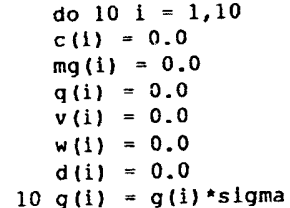

100 format $\left(/ x^{\prime}\right.$ 'covariance matrix' $/ 1$,

$\star f 10.4,3 x, f 10.4,3 x, f 10.4,3 x, f 10.4 / 1 /$,

- $\mathrm{f} 10.4,3 \mathrm{x}, \mathrm{f} 10.4,3 \mathrm{x}, \mathrm{f} 10.4,3 \mathrm{x}, \mathrm{f} 10.4 / / /$,

$*\{10.4,3 x, f 10.4,3 x, 110.4,3 x, 110.4 / 1 / 4$

*f $10.4,3 x, f 10.4,3 x, f 10.4,3 x, f 10.4)$

c model resolution matrix

$m g(1)=v(1) * c(1)+v(2) * c(2)+v(4) * c(4)+v(7) * c(7)$

$m g(2)=v(1) * c(2)+v(2) * c(3)+v(4) * c(5)+v(7) * c c(8)$

$m g(4)=v(1) * c(4)+v(2) * c(5)+v(4) * c(6)+v(7) * c(9)$ 
$m g(5)=v(2) * c(4)+v(3) * c(5)+v(5) * c(6)+v(8) * c(9)$ $m g(6)=v(4) * c(4)+v(5) * c(5)+v(6) * c(6)+v(9) * c(9)$ If (1 a.eq.6) go to 2

$m g(7)=v(1) * c(7)+v(2) * c(8)+v(4) * c(9)+v(7) * c(10)$ $m g(8)=v(2) * c(7)+v(3) * c(8)+v(4)=c(9)+v(7) * c(10)$ mg $(10)=v(7) * c(7)+v(\theta) * c(\theta)+v(9) * c(9)+v(10) * c(10)$ 2 write $(12,102)$

mg (1), mg (2), mg (4), mg (7)

*mg (4), mg (5), mg (6), mg (9).

*mg (7), $\mathrm{mg}(8), \mathrm{mg}(9), \mathrm{mg}(10)$

102 format $/ /$, model resolution matrix'//,

*f $10.4,3 x, f 10.4,3 x, f 10.4,3 x, f 10.4 / 1 /$

f $10.4,3 x_{1}, 110.4,3 x_{1}, 110.4,3 x, f 10.4 / / 7$

\& $10.4,3 x, f 10.4,3 x, f 10.4,3 x, f 10.4)$

c data resolution mat $r i x$

$w(1)=g(1) * v(1)+g(2) * v(2)+g(4) * v(4)+g(7) * v(7)$ $w(2)=g(1) * v(2)+g(2) * v(3)+g(4) * v(5)+g(7) * v(8)$ $w(4)=g(2) * v(4)+g(2) * v(5)+g(4) * v(5)+g(7) * v(9)$ $w(5)=g(2) * v(4)+g(3) * v(5)+g(5) * v(6)+g(8) * v(9)$ $w(6)=g(4) * v(4)+g(5) * v(5)+g(6) * v(6)+g(9) * v(9)$ if (ia.eq. 6$)$ go to 3

$w(7)=g(1) * v(7)+g(2) * v(8)+g(4) * v(9)+g(7) * v(10)$ $w(8)=g(2) * v(7)+g(3) * v(8)+g(5) * v(9)+g(8) * v(10)$ $w(9)=g(4) \cdot v(7)+g(3)-v(8)+g(5) \times v(9)+g(\theta)=v(g)+g(g)=v(10)$

$3 d(1)=w(1) * g(1)+w(2) * g(2)+w(4) * g(4)+w(7) * g(7)$ $d(2)=w(1) * g(2)+w(2) * g(3)+w(4) * g(5)+w(7) * g(8)$ $d(3)=w(2) \cdot g(2)+w(3) \cdot g(3)+w(5) \cdot g(5)+w(8) \cdot g(8)$ $d(5)=w(2) * g(4)+w(3) * g(5)+w(5) * g(6)+w(8) * g(9)$ $d(6)=w(4) * g(4)+w(5) * g(5)+w(6) * g(6)+w(9) * g(9)$ if (ia.eq.6) go to

$d(7)=w(1) * g(7)+w(7) * g(8)+w(4) * g(9)+w(7) * g(10)$ $d(8)=w(2)=g(7)+w(3) \cdot g(8)+w(5) \cdot g(9)+w(8) \cdot g(10)$ $d(9)=w(4) * g(7)+w(5) * g(8)+w(6) * g(g)+w(9) * g(10)$ $d(10)=w(7) * g(7)+w(8) * g(8)+w(9) * g(9)+w(10) * g(10)$

4 write $(12,103)$

* $d(1), d(2), d(4), d(7)$

$\therefore d(2), d(3), d(5), d(8)$ $* d(7), d(8), d(9), d(10)$

103 format. $/, \cdot$ data resolution mat $r i x^{\prime} / /$,

f $10.4,3 x, f 10.4,3 x, f 10.4,3 x, f 10.4 / 1 /$

*f10.4, 3x, f10.4, 3x, f10.4, 3x, f10.4///

$* f 10.4,3 x, f 10,4,3 x, f 10,4,3 x, f 10,41$

95 contidence limits, see Menke pg.2

$\operatorname{ccot}=\operatorname{sqrt}\left(-20^{*} q(1) * a \operatorname{alog}(0.05 * \operatorname{sqrt}(2.0 * p 1) * \operatorname{sqrt}(q(1))\right.$ cph1 $=\operatorname{sqrt}(-2 . * q(3) * a \operatorname{alog}(0.05 * \operatorname{sqrc}(2.0 *$ p1) *sqrt (q(3))1) $c l a m=\operatorname{sqrt}(-2 . * q(6) * a \operatorname{ag}(0.05 * \operatorname{sqrt}(2.0 * p) * \operatorname{sqrt}(q(10)))$

c

error ellipse (see Flinn (1965) Revs. Geophy. V 3, N 1)

c

$u 1(1)=q(3)$ $u 1(3)=q(6)$

d, ldgt, dd1, dd2, ler)

cation of axis

$a p h=\operatorname{atan} 2(2 . \star d(2), d(1)-d(3)) / 2$

if (aaph.1t.0.) aaph $=\mathrm{aaph}+\mathrm{piz}$

c constants of ellipse, normallzed for kllometers

aa $=d(1) * \cos (a a p h) * * 2+2 . * d(2) * \sin (a a p h) * \cos (a a p h)$

\& $\quad+d(3) * \sin (a a p h) * 2$

$b b=d(1) * \sin (a a p h) * * 2-2 * * d(2) * \sin (a a p h) * \cos (a a p h)$

\& $\quad+d(3) * \cos (a a p h) * * 2$

c probility from F statistic: see CRC math tables p. 273$$
\mathrm{npl}=\mathrm{np1}+\mathrm{df}
$$

90 percent confidence

if (npl.qe.3..and.npl.1t.4.) $\quad \mathrm{f}=5.46$

if (npl.ge.4...and.npl.1t.5.) $\quad f f=4.32$

if (npl.ge.5..and.npl.1t.6) $f f=3.78$

if (npl.ge.6...and.npl.1t.7) $\quad f f=3.46$

If (npl.ge.7... and.npl.1t.8) ff $=3.26$

if (npl.ge.8...and.npl.1L.9) $\quad f f=3.11$

if (npl.ge.

if (npl.gt.15.and npl 10.30.$)$ ff $=2.67-(n p 1-16) * 0.012$

If (npl gt 30 and $n p 1.60 .6 \mathrm{ff}=2.19-(\mathrm{npl} 1-30) * 0.0033$

if (npl gt 60 and npl.1e.120.) ff $=2.39-(\mathrm{npl}-60) * 0.00067$

if (npl.gt.120...and.npl.1e.200.) ff $=2.35-(n p 1-120) * 0.0005$ ff $=2.30$

95 percent confidence

if (npl.ge.3...and. npl.1t.4) if (npl.ge. 4 ... and. npl. 1t.5) if ( $\mathrm{npl}$.ge.5... and.npl.1t.6) If (npl.ge.6...and. npl. 1t. 7 ) If (npl.ge. If if (npl.ge.10. and npl.le. 15 .) ff $=4.10-($ npl $1-10) * 0.07$ If (npl at 30.

if (npl.gt.60.. and.npl.le.120.) ff $=3.15-(n p l-60) * 0.0013$ if (npl.gt .120.. and. np1.1e.200.) ff $=3.07-(n p 1-120) * 0.0009$ if (npl.gt .200.)

$f f=9.55$

$f f=6.94$

$f f=5.79$

ff $=4.74$

$f=4.46$

$f f=4.26$

percent

If (npl.ge.3...and.npl.1t.4) ff $=16.04$

if (npl.ge.4...and.npl.it.5) $\quad f f=10.65$

if (npl.ge. ...and.npl.it.6) $\mathrm{ff}=8.43$

If (npl.ge.6. and

if (nol ge 8 and $n p l$ it 9) $f f=6.06$

if $f f=5.71$

if (npl ge. 10 . and.ripl.1e.15.) $\mathrm{ff}=5.46-(\mathrm{npl} 1-10) * 0.115$

If (npl.gt.15...and.npl.le.30.) ff $=4.69-(n p 1-16) * 0.034$

If (npl.gt. 30.. and.npl.1e.60.) ff $=4.18-(n p 1-30) * 0.0083$

If (npl.gt.60.. and.npl.1e.120.) ff $=3.93-(n p 1-60) * 0.0022$ if (npl.gt. 200. )

ff $=3.60$
I

99.0 percent confidence 


\section{hutchs}

rslmtx.f

If (npl.ge.3...and.npl.1t.4)

$\mathrm{ff}=30.82$

1 f (npl.ge. 4 ... and. npl. it . 5 )

if (npl.ge.5... and. npl.1t.6)

if (npl.ge.6... and.npl. lt.7)

1 (npl.ge. 7 . and. npl.1t.8)

if (npl.ge. 9 and npl. it . 10 )

if (npl.ge. 15 .. and.npl.1e.15.) $\quad$ ff $=7.56-(n p 1-10) * 0.2$

if (npl.at.30..and.npl.le.60.) $\mathrm{ff}=5.39-(\mathrm{npl}-30) * 0.0137$

if (npl.gt.60...and.npl.1e.120.) ff $=4.98-(n p 1-60) * 0.0032$

$\begin{array}{ll}\text { if (npl.gt.60..and.npl. } & \text { (npl.gt.120..and.npl.1e.200.) ff }=4.79-(\mathrm{npl} 120) * 0.0023 \\ \text { if (npl.gt.200.) } & \text { ff }=4.61\end{array}$

$f f=4.61$

c equation for ellipse

c

$c 22=f f * 2 * s i g m$

c semiaxes of the ellipse in $\mathrm{km}$, axis in degrees from north

c

$$
\begin{aligned}
& \text { e11 }=\text { sqrt }(c 22 * 2 / a a) * 111.12 \\
& \text { e12 }=\text { sqrt }(c 22 * 2 / b b) * 111.12 \\
& \text { axe }=\text { aaph/dr }
\end{aligned}
$$

retur 
C character*2 pha1, pha2, bstat*4 include ' regloc. cmn

$a k=0.0$

oot $=0.0$

ddkm $=0.0$

If (nopha 2 2 and nbz eq 2) go to 1003

if (apha

bstat = stat (1)

1 do $1001=1$, noph

astat $=$ stat (i)

if (astat ne.bstat) go to 103

14 If (pha (1).eq.bz.or.iwt (i) .eq.4.or.pha(i).eq.' ') go to 100 phal $=$ pha(i)

at $1=$ at (1)

do $20 \mathrm{k}=1$, nopha

if (stat (k) eq.ast at and pha (k) eq. bz) go to 21

if

go to 100

$21 \mathrm{bzz}=\mathrm{baz}(\mathrm{k}) * \mathrm{dr}$

if (baz (k).gt.180.) bzz = piz - bzz

10 lf (astat

$11 j=j+1$

if (jhat (j) ne bz and iwt (j).ne.4.and.stat (j).eq.astat) yo to 13 go to 11

13 pha2 = pha $\{j\}$

at $2=$ at $(j)$

$c$ pn and $\mathrm{sn}$

if (pha1.eq.pn.and.pha2.eq.sn.or.pha1.eq.sn.and.

6 phaz.eq.pn) the

if (depth.le.z2

2) $d k m=f 1(v s 3, v p 3) \cdot(a b s(a i 2-a t))-(p o-1$.$) .$

\& $f(2 \cdot * 22$-dept $h, v p 3, v p 1)-(p o-1,) \cdot\{1) \cdot(z 3-z 2), v p 3, v p ?)$

if (depth.gt. $f(z 2, v p 3, v p 1)-(p o-1,) \times f(2, * z 3-z 2-d e p t h, v p 3, v p 2)\}$

if (depth.le.z2) $\mathrm{tt} 1=\mathrm{dkm} / \mathrm{vp} 3+\mathrm{f}\left(2 .{ }^{*} \mathrm{z} 2\right.$-depth, vp $\left.\left.3, v p\right)\right\}$

$\& f(2 . *(z 3-22), v p 3, v p 2)$

If (depth.gt.z2) tt $]=d \mathrm{~km} / v p 3+\{(z 2, \mathrm{vp} 3, \mathrm{vp} 1)$

$\& \quad\left[\left(2, z^{*} 3-z 2-\right.\right.$ dept $\left.h, v p 3, v p 2\right)$

if (pha1.eq.pn) ot $=a t 1-t t$

I (pha2.eq.pn) endif

c pg and lq 1 (pha1.eq.pg.and.pha2.eq.1q.or.phal.eq.1g.and.

if (pha1.eq.

d pha2.eq.pg) then $=\mathrm{f}$ (v)

$[t]=\mathrm{dkm} / \mathrm{vpl}$

if (phal.eq.pg) ot $=a t l-t t$

if (pha2.eq.pq) ot $=a t 2-t t$

endif

c pn and pg

(1) pha1.eq.pn.and.pha2.eq.pg.or.pha1.eq.pg.and.

\& pha2.eq.pn) then

If (depth.le.z2) dkm $=f 1$ (vp1, vp 3)*(abs (at 2-at 1)+

(12.*22-depth,vp3,vpl) + $(2$. * (23-z2),vp3,vp2n

If (depth.gt.z2) d $\mathrm{f}(2, * 23-22-d$,

$\mathrm{tt} 1=\mathrm{dkm} / \mathrm{vpl}$

If (phal.eq.pq) ot $=a t 1-t t 1$ if (pha2.eq.pg) ot $=a t 2-t t 1$

endif

if (pha1.eq.pn.and.pha2.eq.1q.or.phal.eq.1g.and.

$\&$ pha2.eq.pn) then

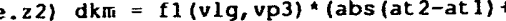

\& $f(2, * z 2-d e p t h, v p 3, v p 1)+f(2 . *(z 3-z 2), v p 3, v p 2:)$

if (depth.gt.22) dkm = f1(vlg, vp3)* (abs (at 2-at 1$)+$

- $f(z 2, v p 3, v p 1)-f(2, * z 3-z 2-d e p t h, v p 3, v p 2)$

if (phal .eq.ig) ot $=a t \cdot 1-t t$

if (pha2.eq.lg) ot $=a t 2-t t$

endif

sn and $\mathrm{pg}$

(pha1.eq.pg.and.pha2.eq.sn.or.phal.eq.sn.and.

pha2.eq.pg) then

(depth. le.22) dkm $=f 1(v s 3, v p 1) *(a b s$ (at 2-at1) -

pot

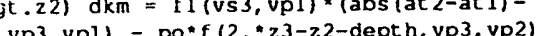

$t t 1=\mathrm{dkm} / \mathrm{vp} 1$

if (phal.eq.py) ot = at 1 - t t

if (pha2.eq.pq) ot $=a t 2-t t$

endif

sn and 19

if (pha1.eq.sn.and.pha2.eq.19.or.pha1.eq.1g.and.

(phaz.eq.snl then

po = vp3/vs3

if (depth. le.22) $\mathrm{dkm}=f(1(v 1 q, v s 3) *(a b s(a t 2-a t 1)+$

if $\left\{(2, * 22\right.$-dept $\left.h, v p 3, v p 1)+p o^{*} f\left(2 *^{*}(z 3-z 2), v p 3, v p 2\right)\right)$

$\mathrm{t} t \mathrm{l}=\mathrm{dkm} / \mathrm{vlg}$

if (phal.eq.1g) ot $=$ at $1-t t$

if (phaz.eq.ig) ot $=a t 2-t t$

endif

$a k=a k+1$.

$\mathrm{ddkm}=\mathrm{ddkm}+\mathrm{dkm}$

oot $=0 o t+o t$

go to 11

101 bstat = stai $(\mathrm{i}+1)$

go to ion

0.1 go lo 102

bstat = astat

100 cont inue

ff lak.eq. 0.0 ) ret urn

102 if (ns.ge.2) irtn $=400$

$\mathrm{dkm}=\mathrm{ddkm} / \mathrm{a}$

ot $=$ oot $/ a k$
delta $=\mathrm{dkm} \cdot \mathrm{dr} / 111.12$

$r p h 1=a \ln (\cos (b z z) * \sin (d e) t a) * \cos (r t(j j))+$

$\cos (d e l t a) * \sin (r t)(j j))$

(r) 1$) * \sin (r t(j j))$

anging

rlam $=$ acos (angl/ang 2 )

if $(\mathrm{baz}(k)$ ge.180.) rlam $=\mathrm{rl}(j \mathrm{j})-\mathrm{rlam}$

if (baz $(k) .1 t .180$.$) rlam =r l(f j)+$ rlam

arlat $=\tan (\mathrm{rph}))$

arlat =atan (arlat/gc)

$\mathrm{dphi}=\mathrm{arlat} / \mathrm{dr}$ 
hs $=1 \mathrm{f} 1 \times(0 \mathrm{t} / 3600$.

$\min =1 \mathrm{f} 1 \times($ (ot $-\mathrm{float}(\mathrm{ins} * 3600)) / 60$.

sec * ot-float (1hs*3600+min*60)

if (ns.ge.2.) return

(Ins $-(1 \mathrm{hs} / 10) * 10+48) / / \mathrm{char}(\mathrm{min} / 10+48)$

(/Char (Min- (Min/10)*10+48)

write $(12,313)$

13 format ('algebraic location with backazimuth from one station') if (fxt .eq.1.) then

dphi $=$ flat

dlam = flon

depth $=$ th

ot $=f l o a t(((i f h s * 60)+i f m l n) * 60)+f s e c$

iyr $=i f y r$
idy $=$ ifdy

$i d y=1$ fdy

315 format ('location fixed")

endif

do $1101=1$, nopha

if (pha (i).eq.bz.or.stat (i).ne.bstat) go to 110

call Lray(i)

that (i) $=$ ot + thtt (i)

$\operatorname{rrs}(i)=$ at (i) - that (i)

110 cont i nue

go to 1001

314 rormat $(10$ arthyuake is in mant le, cant ger algobrats solutione go to 1001

c two station algebric solution for two backazimuths

1003 wrile $(12,312\}$

312 format ('algcbraic location with backazimuth trom two stations' 


\section{arvtim.f}

calculates hrs mns sec of origin time

chival cines ate created in the array "at" as secs from origin time.

arriliest arrival time is called "ot" and all secs from origin time.

subrout ine arvitim

Include 'regloc.cmn'

if lot gt $124 \cdot 3600.11$ go to

if (ot.1t.0.0) yo to 10

go to 20

10 idy $=1$ idy -1

f $(1 d y \cdot 1 t .1)$ iyr - iyr -

if(ldy.1t.1) Idy $=365$

do $30 \mathrm{i}=1$, nopha

at (i) = at (i) $+24 . * 60.60$

go to 20

If (11dy.gt .365) iyr $=1 y x+1$

if (idy.gt.365) idy $=1$

do $25 \mathrm{i}=1$, nopha

25 at (1) = at (1) $-24, * 60, * 60$

ot - ot $-24 .=60 \cdot 960$.

20 ins - iffix/ot/3600.)

$m 1 n=1$ fixilot $-f 10 a t($ ins $\cdot 3600)) / 60$.

sec $=$ ot $-\left\{10 a t\left(i n s \cdot 3600+m^{*} \cdot 60\right)\right.$

return 
program baz

c disbaz azimuth calculations with positive for west and north

c disbaz azimuth calculations with posttive for east and south

character ans*3, ans $1 * 3$, ans $2 * 1$

data ans1, ans $21^{\prime}$ 'yes', ' $\mathrm{y}^{\prime}$

$\mathrm{dr}=\mathrm{p} i \mathrm{i} / 360.0$

$\mathrm{gc}=(1 .-1.1297.) \cdots 2$

const $=57.2957795$

1 print ".

print $\because "$

print, longitude $-179<<180$ positive for west

print $\because$ latitude $-90<<90$ positive nort

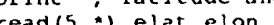

read $(5, *)$ slat, slon

elat $=$ at an (gc.tan (elat $/$ const)

elonirelon/const

slat $1=\operatorname{atan}\left(g c^{\circ} \tan\right.$ (slat/const)

sloni=sion/const

elta $=\operatorname{acos}(\sin (e l a t)) \cdot \sin (\operatorname{siat} 1)+\cos (e l a t)) * \cos (\operatorname{siat} 1) * \cos ($ de 10$))$ baz $11=(\sin (e l a t))-\cos ($ delta $) \cdot \sin (\operatorname{siat} 1)) /(\sin (\operatorname{delta}) \cdot \cos ($ slat 1$))$

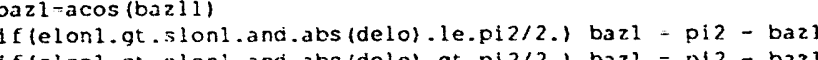

作

belo = sionl - c!on

baz22-sin (be 10$) \cdot \cos (e l a t 1) / \sin ($ detta)

baz2 $=\operatorname{asin}(b a z 22)$

If (slat l. le.elat 1) go to 10

If (belo.gt.-olz.and.belo.le. -3

If (belo.gl.-3..pi2/4..and.belo.1e.-p12/2.) go to 10

if (belo.ql. -pi2/2 ...and.bcio.le. -pi2/4.) baz2 = pi2 + bazz

If (belo.gt. - plzth . and belo.le.

if (belo at pi2/1 and belo.le pi2/2.1 go to 10

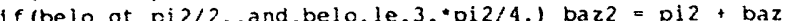

If (belo.gl.3."pi2/4...and.belo.1e.pil) baz2 = piz/2. - baz2

10 ir (bazz.1t 0,) bazz-piz + bazz

bazl-bazl/dr
baz $2=$ ba $22 / d r$

bcz=atan2 (baz22, baz.11)

baz $3=b c z / d$

If (baz3.1t.0.) baz3 $=$ baz3 +360

$a a=-\sin (\operatorname{sion} 1-c \mid 0 n 1) * \sin (\mathrm{elat} 1) * \cos (\operatorname{siat} 1) * \cos (b c z) \cdots 21$

Gisinielati) - sin (slati)

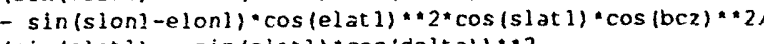

S $(\sin (e) a t))-\sin (\operatorname{siat} 1) * \cos (\mathrm{delt} a))$

delt $a=d e 1 t a * 111.12 / d x$

101 format $(6,1)$ delta, bazl, baz2, baz3, aa, bb

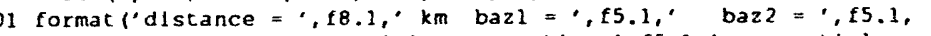
8. baz3 =', f5.11,'partial w.r.t. ph1 - ',f5.1, partial w.r.t. lambda $=,, \mathrm{f} 5.11$ goto 1 
c This rout ine reads in a list of previously located earthquakes c This routine reads in a list of previously located

c subroutine cat 100

c subrout ine to read a list of prevlously located events c purpose:- to add a new event to a previous 11 st

c include 'regloc. cmn'

c open $\left(13, f i l e=\right.$ summary' $^{\prime}$

read $(13,109$, end $=120)$

109 format (//)

nsum $=0$
format (a9, $1 x, f 5.2,2 x, f 7.3,2 x, f 8.3,3 x, f 5.2,2 x, 12,2 x, f 6.2,1 x, f 6.1,4 x$ \&f $5.1,4 x, f 6.1\}$

Sf $5.1,4 x, f 6.1)$
do $105 i=1,1000$

read (13,110, end=120) summ (i), asec (1), adphi (1), adlam(i),

readepth(i), nss (i), sigma (i), elll (i), axel (i), ell? (i)

105 nsum $=$ nsum +1

120 close (13)

return
end 
旁

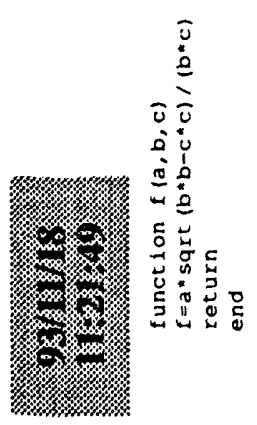


funct ion $f 1(a, b)$

$f 1=a b s(1 . /(1 . / a-1 . / b)$ return 


\section{0,3 .

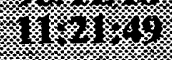

c This routine writes the output of each 1teration of $c$ the rout ine locate.

c subroutine iterout

c include 'regloc.cmn'

yr-char $(\mathrm{iyr} / 10+48) / / \operatorname{char}(1 \mathrm{yr}-(1 \mathrm{yr} / 10) * 10+48)$

$1 d y y=1 d y / 100$

idyyy $=i d y-i d y y^{*} 100-i d y y * 10$

$\mathrm{hm}=\mathrm{char}(\mathrm{ihs} / 10+48) / / \mathrm{char}(\mathrm{hhs}-(\mathrm{hhs} / 10) * 10+48) / / \operatorname{char}(\mathrm{m} / \mathrm{n} / 10+48)$ //char $(\min -(\min / 10) * 10+48)$

write $(12,302) y r$, dy, hm, sec, dphi, dlam, depth

write $(6,303)$ yr, dy, hm, sec, dphi, dl am, depth, rms

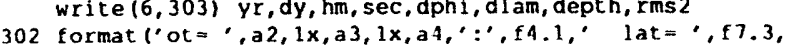

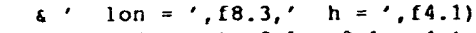

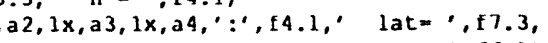

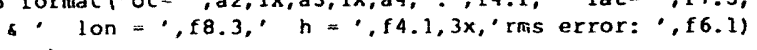
recur

\section{hutchs \\ iterout.f}




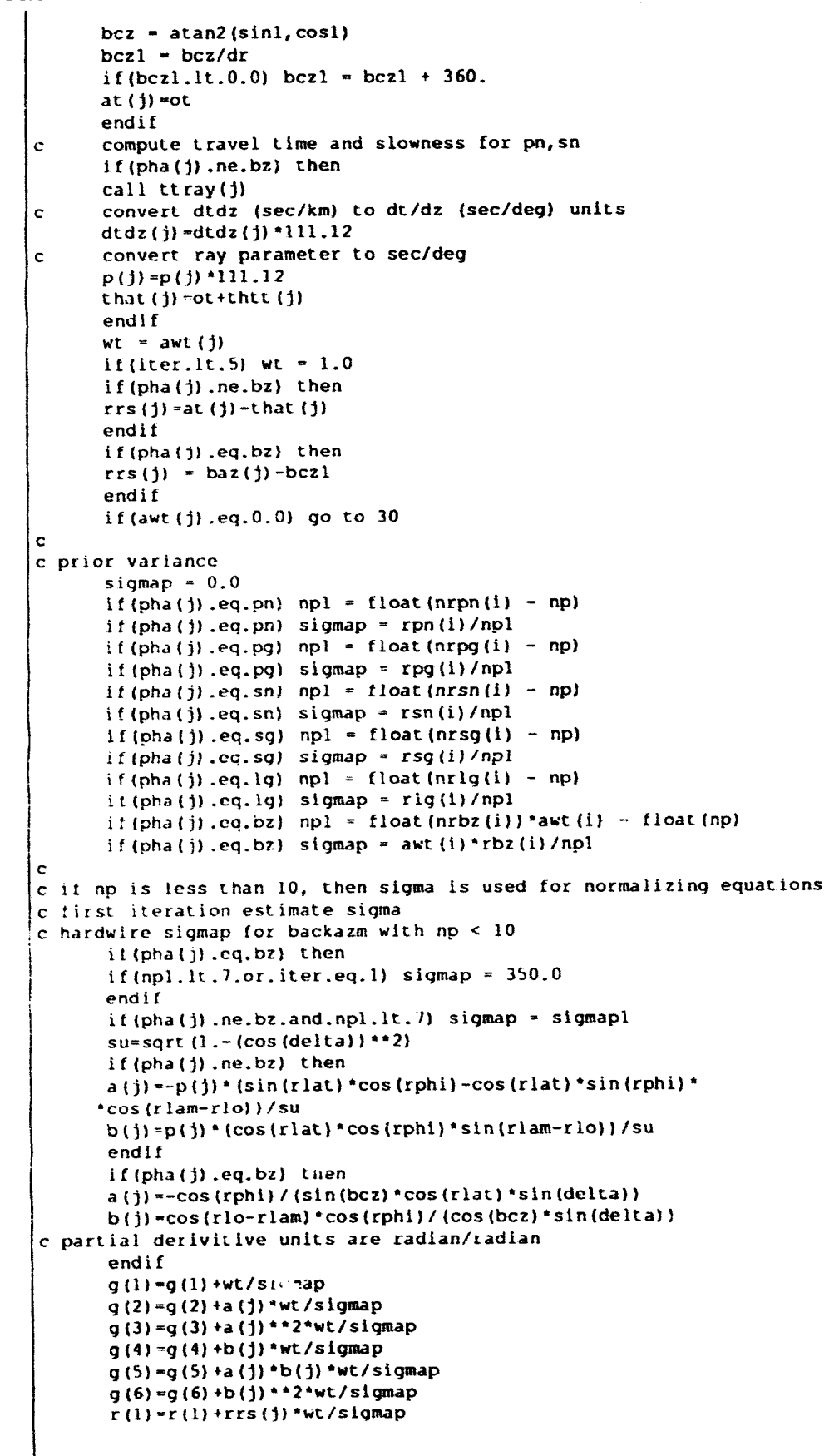

wt $=$ awt (f)

if (awt (j) .eq. 0.0 ) go to 30

sigmap $=0.0$

nn(1) - np)

(f) (pha (j).eq.pg) sigmap $=\mathrm{rpg}(1 / / \mathrm{npl}$

f (pha (j).eq. sn) sigmap $=\mathrm{rsn}(\mathrm{i}) / \mathrm{npl}$

if (pha (j).eq.sg) npl = float (nrsg(i)

if (phat() .eq. lg) npl = lloat (nxlg(I) -

rlammad
(adlam $\mathrm{dph} 11=\mathrm{rph} 1 / \mathrm{d} r$ anph $=0$. do 20 if (i) l, nopha aakm $=\operatorname{acos}(\sin (\mathrm{rt}(\mathrm{i})) \cdot \sin (\mathrm{rph} i) \cdot \cos (\mathrm{rt}(\mathrm{i})) \cdot \cos (\mathrm{rphi}$ $\left.\left.6^{\circ} \cdot \cos (r) a m-r 1(i)\right)\right)$

distt $=a a k m+111.12 \% \mathrm{dr}$

awt $(j)=0.1 * 200.0 / \mathrm{dist}$

endif

illabs(rrs(j)).1L.S.) yo 1020

it (pha (t). ne.bz) then

6 awif (j)

20 If (awt (j).1t .0 .0$)$ awt (i) - 0.0

anph $=$ anph + awt (j)

if (anph.lt. (float (np))! then

1 If(lwe (i) ne.4.) awt (1) $=1.0$

do $40 \quad i=1$, nost

rlat $=r t(1)$
rlo=rl(i)

$u(1)=\sin (r l a t) \cdot \sin (r p h i)+\cos (r) a t) \cdot \cos (r p h i) \cdot \cos (r l a m-r l o)$ delta $a \cos (u(1))$

dkm=delta 111 .12rar

If (stat (j) .ne.sta(1)) go to 30

If (pha (1).eq.bz) then

$\sin 1--\cos (r p h)) \cdot \sin (r 10-r l a m) / \sin ($ delta)

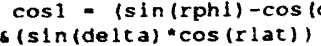




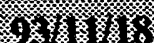

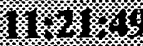

$r(2)=r(2)+a(j)$ arrs $(j) * w t / s$ igmap $r(3)=r(3)+b(j) * r r s(j) * w t /$ sigmap

if (Idc.eq. 1 ) go to 30

$c z=d t d z(j)$

$g(7)=g(7)+\mathrm{Cz}^{*} \mathrm{wt} / \mathrm{sigmap}$

$g(8)=g(8)+a(j) * c z^{*} w^{+}$is igmap

$g(9)=g(9)+b(3) * c z^{*} w c / s i g m a p$

$r(4)=r(4)+c z^{*} r r s(1) * w t / s i g m a p$

30 continue

40 continue factor to diagonal elements of $\mathrm{g}$

$g(1)=g(1)+d a m p$

$g(3)=g(3)+$ dam

$g(6)=g(6)+d a m p$

$q(10)=q(10)+d a m p$

c compute weighted RMS error and degrees of freedom df

c this is used to compute slgmad ldata vartane because weighted variance LJH 7 horg2 change digh to unit var $r$,

doesn't use
d $f=0.0$

$\mathrm{rms}=0.0$

do $60 \mathrm{i}=1$, nopha

if (awt (i) eq. 0.01 go to 60

$a \mathrm{akm}=\operatorname{acos}(\sin (\mathrm{rt}(1)) \cdot \sin (\mathrm{rph}))+\cos (\mathrm{rt}(1)) \cdot \cos (\mathrm{rph})$

$8 * \cos (r 1 a m-r)(i)))$

dist: $=\mathrm{aakm}^{*} 111.12 / \mathrm{dr}$

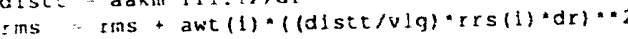

nrms $=$ nrms + awe (i)

endif

if (pha (i).ne.bz) then

(i) $* \operatorname{rrs}(i) \cdots$ ?

nrms = nrms + awt (i)

$c \quad d f=d f+a w t(i)$

$d f=d f$
cont inue

\section{sigmad $=r$}

it (irtn.eq.404) go to 350

if 1 (fxt.eq.1.0) go to 350

solve normal equations

do $50 \quad i=1$, $i$

50 ul (i) - 9 (1)

, inv, idgt, dl, d2, ier

luel imp $(u 1, r, n p, x)$

rms 2 - rms

if (nbz, ne . 0 and.rms2.gt. Ims1.and.iter.gt. 10$)$ go to 1000

$\mathrm{rms} 1=\mathrm{rms} 2$

c convert depth adjustment trom degrees to $\mathrm{km}$

$x(4)-x(4) * 111.12$

$x(4)-x(4), 0)$ write $(12,7)(x(j), j=1, n p), r m s 2$

1f (1dde.

if (idc.ne.0) write $(12,8)(x(3), j=1$, , np), rms 2 ,

6f 4.1, , rms error: $\bullet$, f6.11

8 format $\left(12 x^{\prime}, d t=1\right.$,

$a=\operatorname{abs}(x(1))$

$b b=a b s(x(2))$

cc-abs $(x(3))$

If (bb.1t..001.and.cc.1t..001.and.aa.1t.0.1.and.dd.1t.1.1

\section{hutchs}

\section{locate.f}

sgo to 1000

dph $i 1=d p h i 1+x(2)$

if (dph11.1e.90.) go to 3000

dph $11=180$. -dph 11

go to 4000

3000 If (dphil.ge.-90.) go to 2000

dph $11=-180$. - dphi1
4000 if (dlam.gt.0.) dlam=dlam-180.

If (dl am le 0.) dl am=dlam+180.

2000 dlam-dlam+x(3)

If (dl am.gt.180.) dl am=-360. +d lam

-180.1 dl $a m=360 .+d l a m$

ot $=0 t+x(1)$

dept $h=\operatorname{dept} h+x(4)$

prevent an airquake

If (depth.It.0.) dept $h=0$.

$1 \mathrm{bp}=1$

iter=iter +1

c conver back to geographic degrees

000 rph11 =dph11*dr

rphil $=(\tan (\operatorname{rph} 11)) / q$

$x$ phi $1=\operatorname{atan}(x p h i)$

dphi=rphil/dr

(ind solution ...........)

if liter.gt.1000) go to 350

if (lbp.ea.i) go to 101

if rrms2.1t.5..ur.nst.1t.3) go to 350

arrs $=0.0$

kwe $=0$

do 405 (1) 1, nopha

kwt $=$ kwt +1

if(abs(rts(i)).tt.aers) go to 405

arrs $=a b s(r r s(i))$

405 continue

if (kwt.le.6) go to 350

awt $(j \mathrm{rr})=0.0$

i bp $=1$

iter - 1

write(12,313) stat (jrr),pha(jrr)

313 format $1 /$ stath fleration with phase $, a 4,1 \times$

\& a2, ser to a we'

go to 101

350 df $=$ df - float (np)

return 
subrout ine output

c This routine prints the final output and updates the c file summary

Include ' regloc. cmn'

character alpha*9, bstat*4

c $\quad$ ahr $=$ char (1hs $/ 10+48) / /$ char (ins $-(1 \mathrm{hs} / 10) * 10+48)$ $\mathrm{mn}=\operatorname{char}(\min / 10+48) / / \operatorname{char}(\min -(\min / 10) * 10+48)$ summ $1=\mathrm{yr} / / \mathrm{d} \mathrm{y} / / \mathrm{ahr} / / \mathrm{mn}$

do $4201=1$, nsum

if (summ (i). ne.summl) go to 420

$\operatorname{summ}(i)=\operatorname{summ} 1$

$\operatorname{amn}(1)=m n$

$\operatorname{asec}(i)=\sec$

adlam(l) = dlam

adepth(i) $=$ depth

nss $(i)=n s$

el11(i) $=$ ell

ell2(i) $=$ el2

sigmaa(i) = sigma

$$
\text { go to } 421
$$

20 cont inue

summ (nsum) $=$ summ

$\operatorname{arn}(i)=m n$

asec(i) $=$ sec

acphi(i) $=$ dphi

adepth(i) $=\operatorname{depth}$

nss (i) $=n s$

el11(i) $=$ ell

el12(i) $=$ el2

sigmaa(i) = sigma

301 format $(/, \ldots * * *$ Final Solution ..****) $)$ diy dis res ba

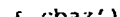

\& $16.1,9 x, f 4.0)$

304 formal $\left(a 4,2 x, a 2,2 x, 11,2 x, f 4.2,2 x, a 4, \prime^{\prime}, f 4.1,1 x, f 5.1,2 x, 6 x, 16.1\right)$

305 format (a4, 2x, a2, 2x, i1, 2x, f4.2, 14x, f6.1,13x, f4.0, 1x, f4.0)

306 format (a4, 2x,a2, 2x, 11,2x, $24,2,13 x, 21 x, 14.0,2 x, 14.01$

07 format ('958 confidence:' $/, 10 x,{ }^{\prime}$ dot $=:^{\prime}, f^{4} .1,2 x,{ }^{\prime}$ dlat $=,, f 5.3, \cdot$ d 1

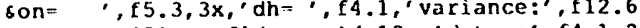

$2 x^{\prime}$ dlat $=\cdot, f 5.3, \cdot d l$ con= $f 5,3,3 x$, vartance:, f6. 21

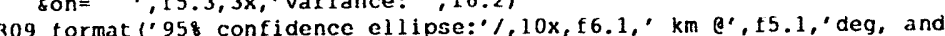
$\left.8^{\prime}, \mathrm{f} 6.1, \mathrm{~km}^{\circ} \mathrm{k}+90 \mathrm{deg}^{\prime}\right)$

310 format ' $^{\prime}$ ot $=', a 2,1 x, a 3,1 x$, aA, $!^{\prime}, f 4.1$,

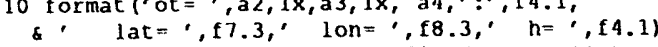

421 write $(12,301)$

if (fxt.eq.1.0) write $\{12,311\}$

write $(12,310)$ yr, dy, hm, sec, dphi, dlam, depth

if (ia.eq.10) write (12,307) ccot, cphi, clam, c22, sign

if (ia.eq.6) write $(12,308)$ ccot, cphi, clam, sigma

write $(12,309)$ ell, axe, el2

write $(12,302)$ ddlta $=100000.0$

do $45 j=1$, nosta

aakm $=\operatorname{accs}(\sin (r t(j)) * \sin (\operatorname{rph} 1)+\cos (r t(j)) * \cos (r p h i)$

$\left.g^{*} \cos (\mathrm{rlam}-\mathrm{r})(j)\right)$

(f) (j) $=12 / d r$

daakm)* $\sin (\mathrm{rt}(j)) / /(\sin (a \mathrm{akm}) * \cos (\mathrm{rt}(j))$

(

if (bcz.1t.0.0) bcz $=\mathrm{bcz}+360,0$

do $401=1$, nopha

if (stat (i). ne.sta (j)) go to 40

$1 \mathrm{f}$ (pha (i).eq.bz) go to 41

c add back in travel time delay to put out arrival time

c find travel t tme delay for station and phase

if (pha(i).eq.pn) tdly $=\operatorname{tpn}(j)$

if (pha (i).eq.sn) tdly $=\operatorname{tsn}(j)$

If (pha (i).eq.pg) tdly $=$ tpg(j)

if (pha (i) eq.lg) tdly $=t \lg (j)$

at (i) $=a t(i)+t d l y$

c ins $=1 \mathrm{fl} \times$ (at (1) $/ 3600$.)

min=char (ihs $/ 10+48) / /$ char $(1 \mathrm{hs}-(1 \mathrm{hs} / 10) * 10+48) / /$ char $(\mathrm{mln} / 10+48)$

8//char $(\min -(\min / 10) * 10+48)$
sec=at (i) - float $($ ihs $* 360,-$ float $(\min * 60)$

if (stat (i).ne.astat) write i12,303) stat (1), pha(1), iwt (1), awt (1),

hm, sec, cdly, dist (j), rrs (1), bez

304) stat (i), pha(i), iwt (i), awt (1). hm, sec, tdly,rrs (i)

if (stat (i)

dist (j), baz (i), bcz
if (stat (i) eq astat) write $(12,306)$ stat (i), pha (i), iwt (i), awt (i),

$\&$ baz(i),bez

12 astat $=$ stat $(i)$

40 continue

5 cont inue

open (15, file $=$ '. . f flgures/ellipses/e' $/ /$ summ1 $/ /$.error'

write $(15,101)$ summ1, agm, ddlta, summ1, summl, summ 1

rormal ('pole 50.0 -96.0\%" "whdow angles $21.021 .021 .021 .0 \%$ cra 103 size 0.7 t t 3 size

8 loc $r^{\prime} /$, ellfiles $e^{\prime, a 9 /}$

'bg sunw sqf' $/{ }^{\prime}$ map' $/$, 'tty'

close (15)

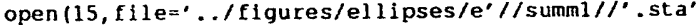

bstat $=$ '

do 551 = 1 , nopha

If istat (1).ne.sta(j) go to 55

bstat = stat (i)

slat $=(\tan (r t(j))) / g$

slat = atan(slat)

slat $=$ slat $/ d r$

write $(15,102)$ sta (j), slat, slon

55 cont inue

02 format (a4, $1 \mathrm{x}, \mathrm{f} 8.4,1 \mathrm{x}, \mathrm{f} 9.4)$ 
close (15)

open $\left(15, \mathrm{flle}={ }^{\prime} .\right.$. / f 1 gures/ell1pses/e $/ /$ summ $1 / / /^{\prime}$.ell'

axe1 $=$ axe -90 .

if (e12.gt.el1) axe $1=a x e 1+90.0$

if (axe1.gt. 360.0) axe1 $=$ axel -360.0

if (axe1.1t.360.0) axel $=a x e 1+360.0$

elp1 $=\operatorname{amax} 1(\mathrm{e} 11, \mathrm{e} 12)$

elp2 = amin1 ell,eld am, elp2, elp1, axel

105 format (f8. 4, 1x, f9.4, 1x, f6.1, 1x, f6.1, 1x, f5.1)

53 close (15)

close (16)

end 
c This averages the back-az's from an Individual station and c creates the phase called "bz"

$$
\text { subrout Ine phabaz }
$$

c location $w: t$ ack-azlmuth if phases only at 0,1 , or 2 stations

include 'regloc. $\mathrm{cmn}$

c nopha1 $=$ nopha

$\mathrm{nbz}=0$
do $10 \mathrm{i}=1$, nosta

avs $=0.0$

avs $=0.0$
bzz $=0.0$

do $20 \mathrm{j}=1, \mathrm{jbz}$

If (stbz (j). ne.sta(i)) go to 20

avs $=$ avs +1.0

bzz $=b 2 z+\operatorname{bazz}(j)$

if (avs.ne.1.0) go to 20

nopha $=$ nopha +1
pha (nopha) $=$ ' $b z^{\prime}$

pha (nopha) $=' b z^{\prime}$
stat (nopha) $=$ sta (1)

iwe (nopha) $=3$

if (nst.ge. 4 . and nophal.ge.6) awt (nopha) $=0.0$

if (nst.ge.4. and.nophal.ge.6) iwt (nopha) - 4

$\mathrm{nbz}=\mathrm{nbz}+\mathrm{I}$

20 cont inue

if (bzz.ne.0.0) baz(nopha) = bzz/avs

10 continue 


\subsection{8}

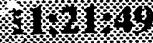

Is rout ine reads arrivals, determine:; if sufficlent info vailable to locate and decides whether or not to use c the back-azimuths estimates

include 'regloc.cmn'

character* 2 bk

open $(11$, file=' phases')

it er $=1$

c read phases

write $(12,201)$
write $(6,201)$

write $(12,202)$

write 112,202 202 format ("' phase information'//,'stat yr day hrmn sec pha wt kly baz bz-err'

作, $11,1 x, 12,13,12,12, f 5.2,2 x, f 5.11$

at relative to days $/$ hrs of flrst arrival

then in subroutine trial ot and at relative to days/00 hrs

nst: no. of stats with at least 1 a

nnst: no. of stats with at least 2 at 1 ba

nbz: number of station with at least
ns: number of stations in location

nopha: total number of phases

astat $=$ '

nopha $=$

$\begin{aligned} \text { nst } & =0 \\ \text { nnst } & =0\end{aligned}$

$n b z=0$

$\mathrm{ns}=0$

$\mathrm{jbz}=0$

$\begin{array}{ll}\text { fbz } & =0 \\ \mathrm{nni} & =0\end{array}$

$n n 2=0$

do $201=1,100$

read (11, 203, end =30) bstat, apha, jwt, iyr, idy, ihs, min, sec, bbaz

if (bstat.eq.astat) go to 10

if (nn1.ge.1) nst $=$ nst +1

if (inn2, (a) 1 ) nbz $=$ nbz +1

if (nn).ge.1.or.nn2.ge.1) ns $=n s+1$

$\mathrm{nn} 1=0$

if (bstat.eq.blank) go to 30

10 if (bbaz.ne.0.0) then

$\mathrm{nn} 2=\mathrm{nn} 2+$

$j b z=j b z+1$

bazz (jbz) = bbaz

$\operatorname{stbz}(j b z)=b s t a t$

$i d y y=i d y / 100$

$i d y y y=\left(1 d y-i d y y^{*} 100\right) / 10$

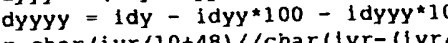

$\mathrm{hm}=\mathrm{char}(\mathrm{ihs} / 10+48) / / \mathrm{char}(\mathrm{hhs}-(\mathrm{hhs} / 10) * 10+48) / / \mathrm{char}(\mathrm{m} / \mathrm{n} / 10+48)$

$8 / / \operatorname{char}(\min -(\min / 10) \star 10+48)$

if (jwt.ne.4) $\mathrm{nnl}=\mathrm{nnl}+$

\section{hutchs \\ rearriv. $f$}

nopha $=$ nopha +1

if (nopha.eq.1) then

iyrl $=$ iyr

$1 \mathrm{dy} 1=i \mathrm{dy}$

ihs $1=$ ins

endif

find travel time delay for station and phas

do $11 j=1,100$

12 if (bstat.eq.sta(j)) go to 12

n) $\operatorname{tdl} y=\operatorname{tpn}(j)$

If (apha.eq.sn) tdly $=\operatorname{tsn}(y)$

if (apha.eq.pg) tdly $=\operatorname{tpg}(j)$

if (apha.eq.sg) $\operatorname{tdly}=\operatorname{tsg}(j)$
if (apha.eq.lg) $\operatorname{tdly}=\operatorname{tgg}(j)$

if(iyr.ne.iyri) idy $=i d y 1+i y r-i y r 1$

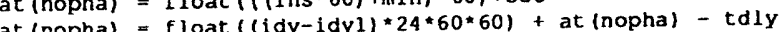

iwt (nopha) = jwt

if (jwt.eq.0) awt (nopha) $=1.0$

if (jwt.eq.1) awt (nopha) $=0.75$

If (fwt eq. 3) awt (nopha) $=0.25$

if (jwt.eq.4) awt (nopha) $=0.0$

pha (nopha) = apha

stat (nopha) = bstat

25 if (bstat.ne.astat) go to 26 , write 112,101$)$ inm sec, apha, jwt if (bbaz ne.0.0. and

at.dly, bbaz,tbz (j)

if (bbaz.eq.0.0.and.apha.ne.bk) writer

if (bbaz.ne.0.0.and.apha.eq.bk) write $(12,103) \mathrm{hm}$, bbaz, tbz (j)

26 if (bbaz.ne.0.0.and.apha.ne.bk) write $(12,104)$ bstat, $y r, d y, h m, s e c$,

S apha,jwt,tdly, bbaz,tbz (j)

if (bbaz eq. 0 0 and apha. ne.bk) write $(12,105)$ bstat, yr, dy, hm, sec,

apha, jwt, tdly
if (bbaz.ne.0.0.and.apha.eq.bk) write $(12,106)$ bstat, yr, dy, hm, bbaz

01 format $\left(12 x, a 4,,^{\prime}, f 4.1,2 x, a 2,2 x, 11,1 x, 55.1,3 x, f 5.1,4 x, \pm 5.1\right)$

$5, i, 4 x, f 5,11$

104 format $(12 x, 24,19 x, 10.1,4 x, 15,1$, f $1,2 x, 2,2 x, 11,1 x, f 5,1,3 x, f 5.1$

$8,4 x, f 5.1)$

for format $(a 4,1 x, a 2,1 x, a 3,1 x, a n, \ldots, f 4,1,2 x, a 2,2 x, 11,1 x, f 5.11$

106 format $\{a 4,1 x, a 2,1 x, a 3,1 x, a 4,19 x, f 5.1,4 x, f 5.1\}$

astat $=$ bstat

if (bbaz 0.0$)$ bazz $(j b z)=b a z z(j b z)-t b z(j)$

20 cont inue

30 iyr $=1 y r 1$

idy $=$ idy 1
ins $=$ i hs 1

if (nopha.eq. 0 . and.nbz.eq. 0 ) then

irtn $=402$$$
\begin{aligned}
& \text { irtn }=\text { return } \\
& \text { ret }
\end{aligned}
$$

endi

(ns.eq.0) the

irtn $=401$

return

ne 0$)$ call phabaz

f (ns.ne.1) go to 31

If (nst.eq. 0 . and.nnst.eq. 0 .and.nbz.eq. 1 ) then irtn $=401$ 
If (nst.eq. 1 .and.nnst.eq. 0 . and.nbz.eq. 1 ) then Irtn $=401$

endif $f$

if (nst.eq.1.and.nnst.eq.1.and.nbz.eq.0) then irtn $=401$

return

endif

If (nst.eq. 1.and.nnst.eq. 0 . and.nbz.eq. 0 ) then irtn $=401$

retur

$1 \mathrm{rtn}=404$

call algeb

31 if (ns.ne.2) go to 32

$1 \mathrm{f}$ (nst.eq.2. and.nnst.eq.1.and.nbz.eq. 0 ) then irtn $=401$

return

if (nst.eq.2.and.nnst.eq. 2.and.nbz.eq.0) then irtn $=401$

return

endif
if (nst.eq. 2 . and.nnst.eq. 0 . and.nbz.eq. 0 ) then $\operatorname{irnn}=40$

endif

if (nst.eq.1.and.nnst.eq.0.and.nbz.eq.1) then irtn $=401$

return

if (nst. eq.1. and.nnst. eq.1.and.nbz.eq.1) then irtn $=401$

endit

if (nst.eq.0.and.nbz.eq.2) write $(12,204)$

204 format ('algebraic location for back-azimuth from two stations' f (nst.eq. 0 .and.nbz.eq. 2$)$ then irtn $=40$

endif

32 if (nst.eq.0) then

ot $=0.0$

$\begin{aligned} \text { dphi } & =45.0 \\ \text { dlam } & =90.0\end{aligned}$

depth $=10.0$

return

endis

call trial

return

end 
subrout I ne sigmp (sigmapl)

include 'regloc.cmn'

for arrivals that do not have enough information

meters solved for

nfe

sigmap $1=0.0$

do $101 \mathrm{i}=1$, nosta

do $201 j=1$, noph

if (awt (j) eq 0.0 ) go to 201

if (stat (j) . ne.sta (1)) go to 20

if (pha (j).ne.pn) go to 202

$n p p=n p p+n r p n(1)$

sigmapl $=$ sigmapl + rpn (i

202 if (pha (j).ne.pg) go to 203 $\mathrm{npp}=\mathrm{npp}+\mathrm{nrpg}(\mathrm{i})$

sigmapl = sigmapl $+\operatorname{rpg}(i)$

go to 201

203 if (pha (j).ne.sn) go to 204 $\mathrm{npp}=\mathrm{npp}+\mathrm{nrsn}(1)$

go 20201

204 if (pha(j).ne.sg) go to 205 $n p p=n p p+n r s g(i)$

sigmapl = sigmapl $+r s g(i)$

$20 j$ if (pha (j).ne.1g) go to 206 $n p p=n p p+n r l g(i)$

sigmapl $=$ sigmapl $+n r l g(i)$

206 if (pha (j).ne.bz) write $(12,110)$ pha(j)

110 format ('phase ', a2,' misident lf led'

clude BAZ in prior variance

01 continue

sigmapl = sigmapl/float (npp - np)

f (npp-np.1t.10) sigmapl $=1.0$

return

end 
c writes the summary file

subroutine sumfile

include ' regloc.cmn'

open $(13$, f 1 le $=$ ' summary' $)$

write $(13,332)$

332 format (

'yrdayhrmn sec lat

\& 95 \& error ellipse'

do $403 i=1$, nsum

if (siqmaa (i).gt.999.) sigmaa (i) $=999$.

If (ell1 (i).gt.9999.) ell1(i) $=9999.9$

if (el12 (i).gt.999.) el12(1) $=9999.9$

If(i.ne.1) write (13,333) sumn(1), asec (i), adphi(i), adlam (i)

adepth(1), nss (1), sigmaa (1), ell (1), axel (1),eli)

if(1.eq.1) write $(13,334)$ summ (1), asec(1), adphi(1), adl am (1)

03 adepthit.

333 format (a9, ': ', r5. 2, 2x, f7. 3, 2x, f8.3, 3x, f5. 2, 2x, 12, 2x, f6.2, 1x

6f $6.1,4 x, f 5.1,4 x, f 6.1)$ f $7,2 x, f 8.3,3 x, f 5.2,2 x, 12,2 x, f 6.2,1 x$,

334 format

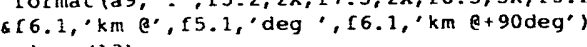

close (13) 
hutchs

table8.f

c creates tex file for table 8

c creates summary and dist.err files first

$$
\text { open (10, file='summary') }
$$

file $x^{\prime}$ dist err':

read $(10,101)$ at $1 \mathrm{~m} 1$ 
c funds a trial location

subroutine trial

include 'regloc.cmn'

dept $h=10.0$

call algeb

if (irtn.eq. 400 and. fxt.ne.1.0) return

att $=1.0 \mathrm{e}+20$

if (pha (i) 1 , nopha

1f (phat (i).eq.bz) go to 10

if (at (i) ge att) go to 10

att $=$ at (i)

$i \mathrm{i}=i$

10 cont inue 1 , nosta

if (sta(j).ne.stat (ii)) go to 20

$\mathrm{dph} 1=r(j) / \mathrm{dr}$

diam $=r l(j) / d r$

20 cont i nue

if (fxt. eq. 1. ) then

dphi $=$ flat

$\operatorname{depth}=\mathrm{fh}$

att $=\mathrm{float}(((\mathrm{ifhs} * 60)+\mathrm{ifmin}) * 60)+\mathrm{fsec}$

iyr $=$ ifyr

$i d y=i f d y$

endit

if (att.ge.0.0) go to 30

ldy $=$ idy $-i$
ins $=23$, if $\mathrm{i} \times(\mathrm{att} / 3600$.

If (idy.lt.il iyx $=$ iyr

If idy.lt.1) idy $=365$

min $=24 \cdot 60 * 60$ tatt $-f(0)(1$ h $* 3600)-f$ loat $(m i n * 60)$

go to 35

30 ins $=$ ifixif(atl-float $(1 \mathrm{hs} * 3600)) / 60$.

$\mathrm{sec}=\mathrm{att}-\mathrm{fl} 10 \mathrm{at}\left\{\mathrm{ihs} * 3600+\mathrm{min} \mathrm{n}^{*} 60\right\}$

ot $=f$ loat $((1 \mathrm{hs} * 60+\mathrm{min}) * 60)+\mathrm{sec}$

35 do $40: i=1$, nopha

40 at (1) $=$ at (1) - att + ot

41 return

end 


\section{ttddel.f}

c determines the travel time partial derivatives

subrout ine ttddel( $j)$

if (pha(j).ne.pn.and.pha(j).ne.sn) go to 50

$\mathrm{vl=vpl}$

if (pha(j).eq.sn) $v 1=v 1 / p o$

(if (pha(j).eq.sn) $v 2=v 2 / p o$

if (pha (j) . eq. $s n) \quad v 3=v 3 / p 0$

c find travel time branch

1st calculate 2 crossover distances, $x c 1, x c 2$

$\mathrm{xcl}=(2 . * 22-$ depth $) \approx \mathrm{s}$

eci $=x+1 / v 1$

$t 12=f(2 * z 2-d e p t h, v 3, v 1)+f(2 * *(z 3-z 2), v 3, v 2)$

$x c 2=(t i 2-t i 1) * v 2 * v 3 /(v 3-v 2)$

$t c 2=t c 1+(x c 2-x c 1) / v 2$

c compute theoretical tts and partial derivatives

$\mathrm{dscl}=\mathrm{dkm}-\mathrm{xc1}$
$\mathrm{dsc2}=\mathrm{dkm}-\mathrm{xc2}$.

If (dkm.gt.xc1 .and. dkm.le.xc2) go to 35

if $(\mathrm{dkm}$.gt.$x \mathrm{c} 2)$ go to 45

sxz=sqrt (akm*2) qo topth**2)

thet

$d t d z(j)=d e p t h /(v) \cdot s \times z)$

go 1040

$p(j)=1 . / v 2$

$\operatorname{dtd} z(j)=f 1(v 2, v 1)$

go to 40

tht $(j)=d \operatorname{se} 2 / v 3+t c 2$

$p(j)=1 . / v$

$\operatorname{dtd} z(j)=f 1(v 3, v 1)$

co to 40

If (pha (j) deq sg) thtt (j) $=$ thtt (j) $*$ po

if (pha (j)

If (pha (j) eq. bz) thtt (j) $=\mathrm{dkm} / \mathrm{v} / \mathrm{q}$

$p(j)=1.0 / \mathrm{vpl}$

if (pha (j).eq.sg) $p(j)=p(j) * p o$

if (pha (j).eq. 1g) $p(j)=1.0 \%$ vig

if (pha (j) .eq.bz) $p(j)=1.0 / v 1 g$

$\operatorname{dtdz}(j)=2.0$ depthr/vpl "sqrt (akm**2+dept $n * 2))$

if (pha (j) .eq.sg) dedz (j) $=$ dedz (j)

if (pha (j) .eq. lal drda (j) $=0.0$

40 return 
c computes travel time, ray parameter, and dtdz

c no raytracer, Pg computed as a direct arrival without depth include 'regloc.cmn'

If (depth.gt . 23) go to 102

c pn and sn

ha (j).eq.pn.or.pha (j) .eq.sn) then

If (depth. le 22) thte (1)

$f(2, *(23-z 2), v p 3, v p 2)$

thtt (j) $=d k m / v p 3+f(z 2, v p 3, v p 1)+$

$f(2 . * z 3-z 2-$ depth, vp3, vp2)

$p(j)=1.0 / v p 3$
If (depth.le.zz) $\operatorname{dtdz}(j)=f(-1, ., v p 3, v p 1)$

If (depth.gt.z2) dtdz(j) $=f(-1,, v p 3, v p 2)$

end if

if (pha (j) .eq.sn) then

po $=$ vp $3 /$ vs 3

thtt $(j)=$ po*thtt (j)

$p(j)=p o * p(j)$

$=p o^{*} d t d z(j)$

c pg

If (pha (j) .eq.pg) then

thtt $(j)=d k m / v p l$

$p(j)=1.0 / v p 1$

$\operatorname{dtd} z(j)=0.0$

$=\lg$

endif

If (pha (j) .eq. lg) then

thtt $(j)=\mathrm{dkm} / \mathrm{vlg}$

$p(j)=1.0 \%$ vlg

$\operatorname{dtdz}(j)=0.0$

c

go to 101

102 write $(12,201)$ format ('earthquake is in mantle, cant get calculate partials')

101 return end 


\section{1:18:}

120250

reads in the velocity and station info

subrout Ine velsta

convention for sign of latitude and longitude:

c latitude + for north and - for south

c longitude + for east and - for west

character alph:80

include 'regloc.cmn'

open (10, fil le $=$ ' st at lons'

c input top of layer, velocity

read $(10, *) 21, z 2, z 3, v p 1, v p 2, v p 3, v s 1, v s 2,4$

po $=1.72$

write $(12,101) 21, v p 1, v s 1, v 1 g, 22, v p 2, v s 2, z 3, v p 3, v s 3, i d c$, po, damp

101 format $("$ earthquake location' $/ \%$, 'velocity model $\%$, top of layer

- puel suel 1guel'

* $3 x, f 4.1,8 x, f 3.1,4 x, f 3.1,5 x, f 3.1 /, 3 x, f 4.1,8 x$

: $\mathrm{f} 3.1,4 \mathrm{x}, \mathrm{f} 3.1 /, 3 \mathrm{x}, \mathrm{f} 4.1,8 \mathrm{x}, \mathrm{f} 3.1,4 \mathrm{x}, \mathrm{f} 3.1 / 1$

"' idc=', 11, ' average vp/vs ratio $=', f 4.2$, ' damping=',$f 10.4$ )

read $(10,102)$ alph

102 format $(2120\}$

write $(12,103)$

c 103 format (// stations prior 95 confidence errors $(\mathrm{Km})$ "travel time delays' $/{ }^{\prime}$ sta lat lon

ssn expgersg erlg tbz tpn tsn tpg tsg tl,

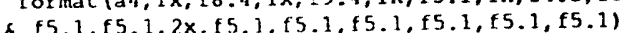

read in station locations, prior square residuals and sample number for $c$ phases recorded at each station.

errors squared, in degrees. erpn,

$c$ ect.: are the sum of travel time errors squared.

$c$ read in travel time delays for each phase at each station. th delays

c are subtracted from traveltimes in rearriv. $f$

nosta $=0$

do $10 \mathrm{i}=1,100$

il sta(i), slat, slo, rbz(i), nrbz(i), ron(i), nrpn(i)

srsn(i), nrsn(i), rpg(i),nrpg(i),rsg(i),nrsg(i),rlg(i),nrlg(i),

stbz(i), tpn(i), tsn(i), tpg(i),tsg(i), tlg(i)

if (sta(i) .eq.blank) go to 11

nosta $=$ nosta +1

$\mathrm{rt}(\mathrm{i})=\mathrm{dr} *$ slat

$r t(i)=g c^{*} \tan (r t(1))$

$\mathrm{rt}(\mathrm{i})=\operatorname{atan}(\mathrm{rt}$ (i)

$r l(1)=\mathrm{dr}^{*} \mathrm{slo}$

vert varlance 95 confidence error for gaussian distribution c compute prior variance

$\operatorname{srbz}=\left(40000.0 / v 1 g^{* * 2}\right) * \mathrm{rbz}(i) * d r^{* * 2}$
$\mathrm{srbz}=\operatorname{srbz} /(\mathrm{nrbz}(1)-\mathrm{np})$

erbz $=\operatorname{sqrt}(-2 * \operatorname{srbz} * a 10 g(0.01 * \operatorname{sqrt}(2.0 * \operatorname{pi}) * \operatorname{sqrt}(\operatorname{srbz}))$

$\operatorname{sxpn}=\operatorname{rpn}(1) /(\operatorname{nrpn}(1)-n p)$

$05 * \operatorname{sqrt}(2.0 * p i) * \operatorname{sqrt}(\operatorname{srpn}))$

$\operatorname{srsn}=r \operatorname{sn}(1) /(\operatorname{srsn}(1)-n p)$

ersn $=\operatorname{sqrt}(-2 . * \operatorname{srsn} * a 10 g(0.05 * \operatorname{sqrt}(2.0 * \mathrm{pt}) * \operatorname{sqrt}(\operatorname{srsn}))$

$\operatorname{srpg}=\operatorname{rpg}(1) /(\operatorname{nrpg}(1)-n p)$

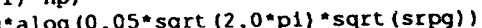

srsg = sqrt $(-2 * \operatorname{srsg} * \operatorname{alog}(0.05 * \operatorname{sqrt}(2.0 * p i) * \operatorname{sqrt}(\operatorname{srsg}))$

\section{hutchs}

velsta.f

$\operatorname{srlg}=\operatorname{rlg}(1) /(n r l g(1)-n p)$
$\operatorname{erlg}=\operatorname{sqrt}(-2 . * \operatorname{srlg} a \operatorname{alog}(0.05 * \operatorname{sqrt}(2.0 * p 1) * \operatorname{sqrt}(\operatorname{srlg})))$

$=$ write $(12,104)$ sta (1), slat, slo, erbz, expn, ersn, erpg, ersq, er lg.

stbz(1), ton(1), tsn(1), tpg(1),tsq(1), tig(i)

10 cont inue 

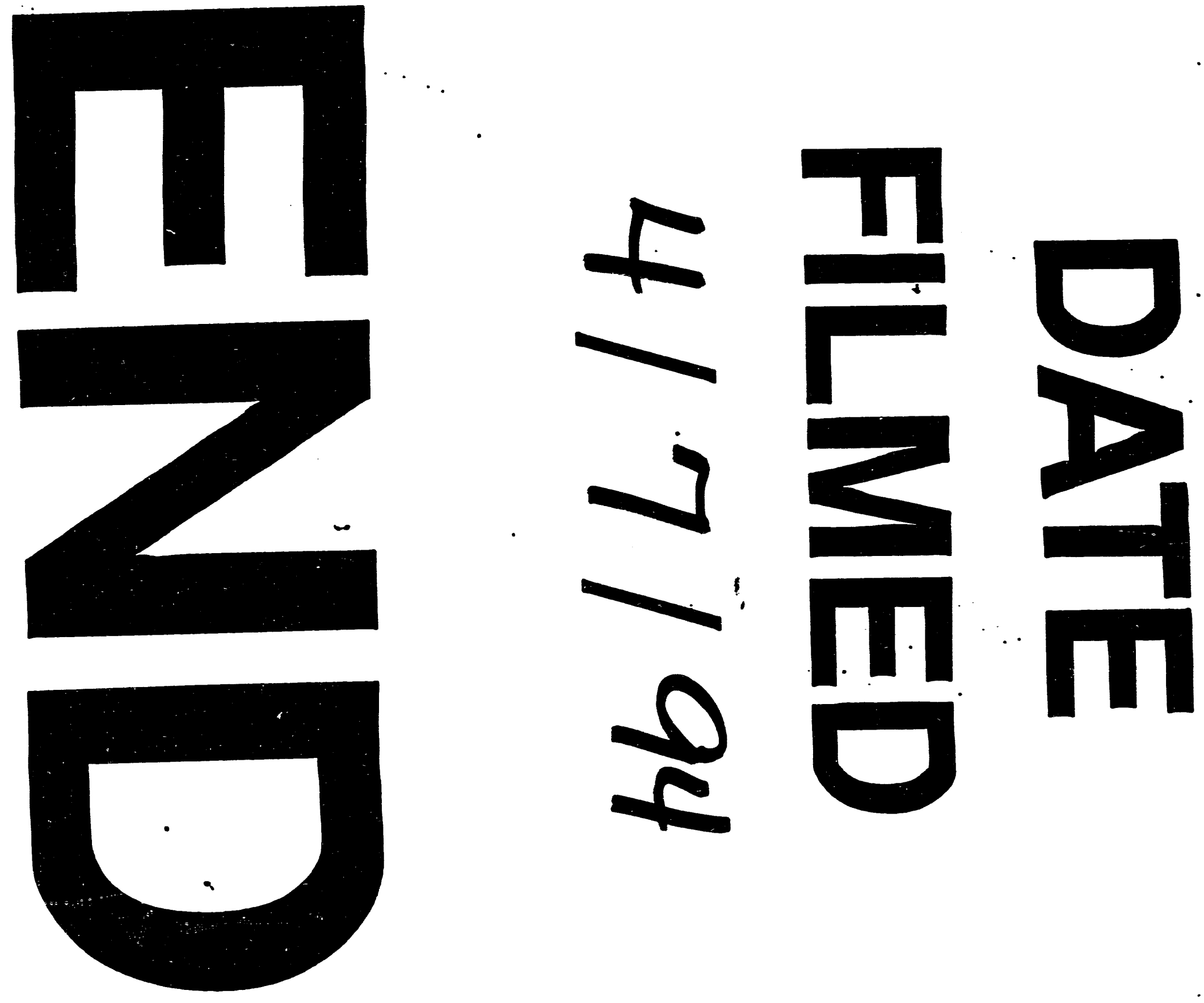
